\title{
Completed Group Algebras of Abelian-by-procyclic Groups
}

\author{
Adam Jones
}

December 3, 2018

\begin{abstract}
Let $p$ be prime, let $G$ be a $p$-valuable group, isomorphic to $\mathbb{Z}_{p}^{d} \rtimes \mathbb{Z}_{p}$, and let $k$ be a field of characteristic $p$. We will prove that all faithful prime ideals of the completed group algebra $k G$ are controlled by $Z(G)$, and a complete decomposition for $\operatorname{Spec}(k G)$ will follow. The principal technique we employ will be to study the convergence of Mahler expansions for inner automorphisms.
\end{abstract}

\section{Contents}

1 Introduction

2 Preliminaries

3 Non-commutative Valuations

4 Central Simple algebras

5 The Extended Commutator Subgroup

6 Construction of Growth Preserving Polynomials

\section{Introduction}

Let $p$ be a prime, $k$ be a field of characteristic $p$, and let $G$ be a compact $p$-adic Lie group. The completed group algebra, or Iwasawa algebra of $G$ with respect to $k$ is defined as:

$$
k G:=\lim _{N \unlhd_{\circ} G} k[G / N] .
$$

We aim to improve our understanding of the prime ideal structure of completed group algebras, which would have profound consequences for the representation theory of compact $p$-adic Lie groups.

\subsection{Background}

In [3, Section 6], Ardakov and Brown ask a number of questions regarding the two-sided ideal structure of $k G$. Several of these have now been answered or partially answered, but a number of them remain open. We hope that this paper will take important steps towards providing an answer to these questions.

First recall some important definitions:

Definition 1.1. Let $I$ be a right ideal of $k G$ :

1. We say that $I$ is faithful if for all $g \in G, g-1 \in I$ if and only if $g=1$, i.e. $G \rightarrow \frac{k G}{I}, g \mapsto g+I$ is injective.

2. We say that $H \leq_{c} G$ controls $I$ if $I=(I \cap k H) k G$.

Define the controller subgroup of $I$ by $I^{\chi}:=\bigcap\left\{U \leq_{o} G: U\right.$ controls $\left.I\right\}$, and denote by Spec ${ }^{f}(k G)$ the set of all faithful prime ideals of $k G$.

Also recall the following useful result [2, Theorem A]: 
Theorem 1.1. Let $I$ be a right ideal of $k G$. Then $I^{\chi} \unlhd_{c} G$, and $H \leq_{c} G$ controls $I$ if and only if $I^{\chi} \subseteq H$.

We will assume throughout that $G$ is $p$-valuable, i.e. carries a complete $p$-valuation $\omega: G \rightarrow \mathbb{R} \cup\{\infty\}$ in the sense of [10, III 2.1.2].

Notation: we say $N \unlhd_{c}^{i} G$ to mean that $N$ is a closed, isolated, normal subgroup of $G$.

One of the main open problems in the subject concerns proving a control theorem for faithful prime ideals in $k G$. To illustrate the importance of this problem, consider the following result ([1, Theorem A]):

Theorem 1.2. Let $G$ be a p-valuable group such that for every $N \unlhd_{c}^{i} G, P \in S p e c{ }^{f}\left(k \frac{G}{N}\right)$, $P$ is controlled by the centre of $\frac{G}{N}$, i.e. $P^{\chi} \subseteq Z\left(\frac{G}{N}\right)$.

Then every prime ideal $P$ of $k G$ is completely prime, i.e. $\frac{k G}{P}$ is a domain, and we have a bijection:

$$
\operatorname{Spec}(k G) \leftrightarrow \coprod_{N \unlhd_{c}^{i} G} \operatorname{Spec}^{f}\left(k Z\left(\frac{G}{N}\right)\right)
$$

Note: This is stronger than the statement given in [1, but it has an identical proof.

This Theorem is the strongest result we have to date concerning ideal classification for $k G$. Using it, we reduce the problem of classifying ideals in the non-commutative ring $k G$ to the simpler problem of classifying ideals in the commutative strata $k Z\left(\frac{G}{N}\right)$.

The result gives a positive answer to [3, Question N] for all groups $G$ for which we have the appropriate control theorem for faithful primes in $k G$, i.e. for all groups $G$ such that $P^{\chi} \subseteq Z\left(\frac{G}{N}\right)$ for $P$ faithful.

It follows from the following result ([1, Theorem 8.4]) that this is true if $G$ is nilpotent.

Theorem 1.3. Let $G$ be a p-valuable group, $P \in \operatorname{Spec}^{f}(k G)$, and suppose that $P$ is controlled by a nilpotent subgroup of $G$. Then $P$ is controlled by the centre of $G$.

We want to extend this result to more general groups, in particular when $G$ is solvable, and thus give a positive answer to [3, Question O].

\subsection{Aims}

In this paper, we will consider certain classes of metabelian groups, i.e. groups whose commutator subgroup is abelian.

A $p$-valuable group $G$ is abelian-by-procyclic if it is isomorphic to $\mathbb{Z}_{p}^{d} \rtimes \mathbb{Z}_{p}$ for some $d \in \mathbb{N}$. The completed group algebras of these groups have the form of skew-power series rings $R[[x ; \sigma, \delta]]$, for $R$ a commutative power series ring.

Our main result establishes the control condition in Theorem 1.2 for groups of this form:

Theorem 1.4. Let $G$ be a p-valuable, abelian-by-procyclic group. Then every faithful prime ideal of $k G$ is controlled by $Z(G)$.

Using Lemma 2.1 below, we see that if $G$ is an abelian-by-procyclic group, and $N$ is a closed, isolated normal subgroup, then $\frac{G}{N}$ is also abelian-by-procyclic, and hence by the theorem, every faithful prime ideal of $k \frac{G}{N}$ is controlled by the centre. Hence we can apply Theorem 1.2 to get a complete decomposition for $S p e c(k G)$.

In particular, if $G$ is any solvable, $p$-valuable group of rank 2 or 3 , then $G$ is abelian-by-procyclic, so again, we can completely determine $\operatorname{Spec}(k G)$.

Most of the work in this paper will go towards proving the following theorem.

Theorem 1.5. Let $G$ be a non-abelian, p-valuable, abelian-by-procyclic group. If $P \in S_{p e c}^{f}(k G)$ then $P$ is controlled by a proper, open subgroup of $G$.

This result is actually all we need to deduce Theorem 1.4 
Proof of Theorem [1.4. Recall from [1, Definition 5.5] that a prime ideal $P$ of $k G$ is non-splitting if for all $U \leq_{o} G$ controlling $P, P \cap k U$ is prime in $k U$.

Fix $G$ a $p$-valuable, abelian-by-procyclic group, and suppose that $P \in \operatorname{Spec}^{f}(G)$ is non-splitting.

Consider the controller subgroup $P^{\chi}$ of $P$. Using [1, Proposition 5.5] and the non-splitting property, we see that $Q:=P \cap k P^{\chi}$ is a faithful prime ideal of $k P^{\chi}$. We know that $P^{\chi}$ is a closed, normal subgroup of $G$, so it follows from Lemma 2.1 below that $P^{\chi}$ is abelian-by-procyclic.

If $P^{\chi}$ is abelian, then it follows from Theorem 1.3 that $P^{\chi}$ is central in $G$, i.e. $P^{\chi} \subseteq Z(G)$ and $P$ is controlled by $Z(G)$ as required. So assume for contradiction that $P^{\chi}$ is non-abelian:

Applying Theorem 1.5 gives that $Q$ is controlled by a proper open subgroup $U$ of $k P^{\chi}$, i.e. $Q=(Q \cap k U) k P^{\chi}$. But $P$ is controlled by $P^{\chi}$ by [2, Theorem A], so:

$$
P=\left(P \cap k P^{\chi}\right) k G=Q k G=(Q \cap k U) k P^{\chi} k G=(P \cap k U) k G
$$

Hence $P$ is controlled by $U$, which is a proper subgroup of $P^{\chi}-$ contradiction.

So, we conclude that any faithful, non-splitting prime ideal of $k G$ is controlled by $Z(G)$. Now suppose that $I$ is a faithful, virtually non-splitting right ideal of $k G$, i.e. $I=P k G$ for some open subgroup $U$ of $G, P$ a faithful, non-splitting prime ideal of $k U$.

Using Lemma 2.1 below, we see that $U$ is $p$-valuable, abelian-by-procyclic, so by the above discussion, $P$ is controlled by $Z(U)$, and in fact, $Z(U)=Z(G) \cap U$ by [1, Lemma 8.4]. Therefore, since $I \cap k U=P$ by 1 , Lemma 5.1(ii)]:

$$
I=P k G=(P \cap k Z(U)) k U k G=(I \cap k U \cap k Z(G)) k G=(I \cap k Z(G)) k G
$$

So since $G$ is $p$-valuable and every faithful, virtually non-splitting right ideal of $k G$ is controlled by $Z(G)$, it follows from [1, Theorem 5.8, Corollary 5.8] that every faithful prime ideal of $k G$ is controlled by $Z(G)$ as required.

So the remainder of our work will be to prove Theorem 1.5, this proof will be given at the end of section 6 .

\subsection{Outline}

Throughout, we will fix $G=\mathbb{Z}_{p}^{d} \rtimes \mathbb{Z}_{p}$ a non-abelian, $p$-valuable, abelian-by-procyclic group, $P$ a faithful prime ideal of $k G$, and let $\tau: k G \rightarrow Q\left(\frac{k G}{P}\right)$ be the natural map. To prove a control theorem for $P$, we will follow a similar approach to the method used in [1].

The most important notion we will need is the concept of the Mahler expansion of an automorphism $\varphi$ of $G$, introduced in [1, Chapter 6], and which we will recap in section 2.

In our case, we will take $\varphi$ to be the automorphism defining the action of $\mathbb{Z}_{p}$ on $\mathbb{Z}_{p}^{d}$.

In section 2, we will see how we can use the Mahler expansion of $\varphi^{p^{m}}$ to deduce an expansion of the form:

$$
0=q_{1}^{p^{m}} \tau \partial_{1}(y)+\ldots .+q_{d}^{p^{m}} \tau \partial_{d}(y)+O\left(q^{p^{m}}\right)
$$

Where $y \in P$ is arbitrary, $\partial_{i}: k G \rightarrow k G$ is a derivation, $q_{i}, q \in Q\left(\frac{k G}{P}\right)$, we call the $q$ 's Mahler approximations. We want to examine convergence of this expression as $m \rightarrow \infty$ to deduce that $\tau \partial_{i}(P)=0$ for some $i \leq d$, from which a control theorem should follow.

Also, for an appropriate polynomial $f$, we will see that we have a second useful expansion:

$$
0=f\left(q_{1}\right)^{p^{m}} \tau \partial_{1}(y)+\ldots+f\left(q_{d}\right)^{p^{m}} \tau \partial_{d}(y)+O\left(f(q)^{p^{m}}\right)
$$

The idea is to compare the growth of the Mahler approximations with $m$ so that we can scale this expression and get that the higher order terms tend to zero, and the lower order terms converge to something non-zero.

One possiblity would be to divide out the lower order terms, which was the approach used in [1]. But an important difference in our situation is that we cannot be sure that the elements $f\left(q_{i}\right)$ are regular, i.e. that 
dividing them out of the expression will not affect convergence of the higher order terms.

In section 3, we will recap how to use a filtration on $k G$ to construct an appropriate filtration $v$ on $Q\left(\frac{k G}{P}\right)$, and introduce the notion of a growth rate function, which allows us to examine the growth with $m$ of our elements $f(q)^{p^{m}}$ with respect to $v$.

We will also introduce the notion of a growth preserving polynomial (GPP), and show how a control theorem follows from the existence of such a polynomial $f$ satisfying certain $v$-regularity conditions.

To prove that a GPP $f$ satisfies these conditions, it is only really necessary to prove that $f(q)$ is central, non-nilpotent of minimal degree inside gr $\frac{k G}{P}$.

Ensuring the elements $f(q)$ are central and non-nilpotent in gr $\frac{k G}{P}$ can usually be done, provided $Q\left(\frac{k G}{P}\right)$ is not a CSA - a case we deal with separately in section 4 using a technique involving diagonalisation of the Mahler approximations.

Ensuring these elements have minimal degree, however, is more of a problem when using the standard filtration on $k G$.

In section 5, we define a new filtration on $k G$ which ensures that we can find a GPP satisfying the required conditions, and we construct such a polynomial in section 6 . This will allow us to complete our analysis and prove Theorem 1.5 .

Acknowledgements: I would like to thank Konstantin Ardakov for supervising me in my research, and for many helpful conversations and suggestions. I would also like to thank EPSRC for funding this research.

\section{Preliminaries}

Throughout, we will use the notation (.,.) to denote the group commutator, i.e. $(g, h)=g h g^{-1} h^{-1}$.

\subsection{Abelian-by-procyclic groups}

Fix $G$ a compact $p$-adic Lie group carrying a $p$-valuation $\omega$. Since $G$ is compact, it follows that $G$ has finite rank $d \in \mathbb{N}$ by [10, III 2.2.6], i.e. there exists a finite ordered basis $\underline{g}=\left\{g_{1}, \ldots ., g_{d}\right\}$ such that for all $g \in G$, $g=g_{1}^{\alpha_{1}} \ldots . g_{d}^{\alpha_{d}}$ for some $\alpha_{i} \in \mathbb{Z}_{p}$ unique, and $\omega(g)=\min \left\{v_{p}\left(\alpha_{i}\right)+\omega\left(\bar{g}_{i}\right): i=1, \ldots ., d\right\}$.

Hence $G$ is homeomorphic to $\mathbb{Z}_{p}^{d}$, and furthermore, it follows from [10, I 2.3.17] that $k G \cong k\left[\left[b_{1}, \cdots, b_{d}\right]\right]$ as $k$-vector spaces, where $b_{i}=g_{i}-1$ for each $i$.

Recall the definition of a $p$-saturated group [10, III.2.1.6], and recall that the category of $p$-saturated groups is isomorphic to the category of saturated $\mathbb{Z}_{p}$-Lie algebras via the log and exp functors [10, IV.3.2.6].

This means that for $G$ a $p$-saturated group, $\log (G)$ is a free $\mathbb{Z}_{p}$-Lie subalgebra of the $\mathbb{Q}_{p}$-Lie algebra $\mathfrak{L}(G)$ of $G$.

Also, recall that any $p$-valuable group $G$ can be embedded as an open subgroup into a $p$-saturable group $\operatorname{Sat}(G)$, and hence $\operatorname{Sat}(G)^{p^{t}} \subseteq G$ for some $t \in \mathbb{N}$ - see [10, III.3.3.1] for full details.

Definition 2.1. A compact p-adic Lie group $G$ is abelian-by-procyclic if there exists $H \unlhd_{c}^{i} G$ such that $H$ is abelian and $\frac{G}{H} \cong \mathbb{Z}_{p}$. For $G$ non-abelian, $H$ is unique, and we call it the principal subgroup of $G$; it follows easily that $Z(G) \subseteq H$ and $(G, G) \subseteq H$, so $G$ is metabelian.

If $G$ is non-abelian, abelian-by-procyclic with principal subgroup $H$, then $G \cong H \rtimes \mathbb{Z}_{p}$. So if we assume that $G$ is $p$-valuable, we have that $G \cong \mathbb{Z}_{p}^{d} \rtimes \mathbb{Z}_{p}$.

Moreover, we can choose an element $X \in G \backslash H$ such that for every $g \in G, g=h X^{\alpha}$ for some unique $h \in H$, $\alpha \in \mathbb{Z}_{p}$. We call $X$ a procyclic element in $G$.

Lemma 2.1. Let $G$ be a non-abelian p-valuable abelian-by-procyclic group with principal subgroup $H$, and let $C$ be a closed subgroup of $G$. Then $C$ is abelian-by-procyclic.

Furthermore, if $N$ is a closed, isolated normal subgroup of $G$, then $\frac{G}{N}$ is p-valuable, abelian-by-procyclic.

Proof. Let $H^{\prime}:=C \cap H$, then clearly $H^{\prime}$ is a closed, isolated, abelian normal subgroup of $C$. 
Now, $\frac{C}{H^{\prime}}=\frac{C}{H \cap C} \cong \frac{C H}{H} \leq \frac{G}{H} \cong \mathbb{Z}_{p}$, hence $\frac{C}{H^{\prime}}$ is isomorphic to a closed subgroup of $\mathbb{Z}_{p}$, i.e. it is isomorphic to either 0 or $\mathbb{Z}_{p}$.

If $\frac{C}{H^{\prime}}=0$ then $C=H^{\prime}=H \cap C$ so $C \subseteq H$. Hence $C$ is abelian, and therefore abelian-by-procyclic.

If $\frac{C}{H^{\prime}} \cong \mathbb{Z}_{p}$ then $C$ is abelian-by-procyclic by definition.

For $N \unlhd_{o}^{i} G$, it follows from [10, IV.3.4.2] that $\frac{G}{N}$ is $p$-valuable, and clearly $\frac{H N}{N}$ is a closed, abelian normal subgroup of $G$.

Consider the natural surjection of groups $\mathbb{Z}_{p} \cong \frac{G}{H} \rightarrow \frac{G}{H N} \cong \frac{G / N}{H N / N}$ :

If the kernel of this map is zero, then it is an isomorphism, so $\frac{G / N}{H N / N} \cong \mathbb{Z}_{p}$, so $\frac{G}{N}$ is abelian-by-procyclic by definition.

If the kernel is non-zero, then $\frac{G}{H N}$ is a non-trivial quotient of $\mathbb{Z}_{p}$, and hence is finite, giving that $H N$ is open in $G$. So $\frac{H N}{N}$ is abelian and open in $\frac{G}{N}$, and it follows that $\frac{G}{N}$ is abelian, and hence abelian-by-procyclic.

Now, fix $G$ a non-abelian $p$-valuable, abelian-by-procyclic group with principal subgroup $H$, procyclic element $X$. Let $\varphi \in \operatorname{Inn}(G)$ be conjugation by $X$, then it is clear that $\varphi \neq i d$.

Recall from [1, Definition 4.8, Proposition 4.9] the definition of $z\left(\varphi^{p^{m}}\right): H \rightarrow \operatorname{Sat}(H), h \mapsto \lim _{n \rightarrow \infty}\left(\varphi^{p^{m+n}}(h) h^{-1}\right)^{p^{-n}}$.

Using [10, IV. 3.2.3] it follows that $z\left(\varphi^{p^{m}}\right)(h)=\exp \left(\left[p^{m} \log (X), \log (h)\right]\right)$, and hence $z\left(\varphi^{p^{m}}\right)(h)=z(\varphi)(h)^{p^{m}}$ for all $m$.

For each $m \in \mathbb{N}$, define $u_{m}:=z\left(\varphi^{p^{m}}\right)$, then it is clear that $u_{m}(h)=u_{0}(h)^{p^{m}}$ for all $g \in G$. So fix $m_{1} \in \mathbb{N}$ such that $u_{0}(h)^{p^{m_{1}}} \in H$ for all $g \in G$, and let $u:=u_{m_{1}}: H \rightarrow H$.

We call $m_{1}$ the initial power, we may choose this to be as high as we need.

\section{$2.2 \quad$ Filtrations}

Let $R$ be any ring, we assume that all rings are unital.

Definition 2.2. A filtration of $R$ is a map $w: R \rightarrow \mathbb{Z} \cup\{\infty\}$ such that for all $x, y \in R, w(x+y) \geq$ $\min \{w(x), w(y)\}, w(x y) \geq w(x)+w(y), w(1)=0, w(0)=\infty$. The filtration is separated if $w(x)=\infty$ implies that $x=0$ for all $x \in R$.

If $R$ carries a filtration $w$, then there is an induced topology on $R$ with the subgroups $F_{n} R:=\{r \in R$ : $w(r) \geq n\}$ forming a basis for the neighbourhoods of the identity. This topology is Hausdorff if and only if the filtration is separated.

Recall from [12, Ch.II Definition 2.1.1] that a filtration is Zariskian if $F_{1} R \subseteq J\left(F_{0} R\right)$ and the Rees ring $\widetilde{R}:=\bigoplus_{n \in \mathbb{Z}} F_{n} R$ is Noetherian.

Zariskian filtrations can only be defined on Noetherian rings, and by [12, Ch.II Theorem 2.1.2] we see that a Zariskian filtration is separated.

If $R$ carries a filtration $w$, then define the associated graded ring of $R$ to be

$$
\operatorname{gr} R:=\underset{n \in \mathbb{Z}}{\oplus} \frac{F_{n} R}{F_{n+1} R}
$$

This is a graded ring with multiplication given by $\left(r+F_{n+1} R\right) \cdot\left(s+F_{m+1} R\right)=\left(r s+F_{n+m+1} R\right)$.

Notation: For $r \in R$ with $w(r)=n$ we denote by $\operatorname{gr}(r):=r+F_{n+1} R \in \operatorname{gr} R$.

We say that a filtration $w$ is positive if $w(r) \geq 0$ for all $r \in R$. 
Definition 2.3. If $R$ carries a filtration $v$ and $x \in R \backslash\{0\}$, we say that $x$ is $v$-regular if $v(x y)=v(x)+v(y)$ for all $y \in R$, i.e. $g r(x)$ is not a zero divisor in $\operatorname{gr} R$. If all non-zero $x$ are $v$-regular we say that $v$ is a valuation.

Note that if $x$ is $v$-regular and a unit then $x^{-1}$ is $v$-regular and $v\left(x^{-1}\right)=-v(x)$.

Examples: 1. If $I$ is an ideal of $R$, the $I$-adic filtration on $R$ is given by $F_{0} R=R$ and $F_{n} R=I^{n}$ for all $\overline{n>0 \text {. If } I}=\pi R$ for some normal element $\pi \in R$, we call this the $\pi$-adic filtration.

2. If $R$ carries a filtration $w$, then $w$ extends naturally to $M_{k}(R)$ via $w\left(\left(a_{i, j}\right)\right)=\min \left\{w\left(a_{i, j}\right): i, j=1, \cdots, k\right\}$ - the standard matrix filtration.

3. If $R$ carries a filtration $w$ and $I \unlhd R$, we define the quotient filtration $\bar{w}: \frac{R}{I} \rightarrow \mathbb{Z} \cup\{\infty\}, r+I \mapsto$ $\sup \{w(r+y): y \in I\}$. In this case, $\operatorname{gr}_{\bar{w}} \frac{R}{I}=\frac{\operatorname{gr} R}{\operatorname{grI}}$.

4. Let $(G, \omega)$ be a $p$-valuable group with ordered basis $g=\left\{g_{1}, \cdots, g_{d}\right\}$. We say that $\omega$ is an abelian $p$-valuation if $\omega(G) \subseteq \frac{1}{e} \mathbb{Z}$ for some $e \in \mathbb{Z}$, and $\omega((g, h))>\omega(g)+\omega(h)$ for all $g, h \in G$.

If $\omega$ is abelian, then by [1, Corollary 6.2$], k G \cong k\left[\left[b_{1}, \cdots, b_{d}\right]\right]$ carries an associated Zariskian filtration given by

$$
w\left(\sum_{\alpha \in \mathbb{N}^{d}} \lambda_{\alpha} b_{1}^{\alpha_{1}} \cdots b_{d}^{\alpha_{d}}\right)=\inf \left\{\sum_{i=1}^{d} e \alpha_{i} \omega\left(g_{i}\right): \lambda_{\alpha} \neq 0\right\} \in \mathbb{Z} \cup\{\infty\} .
$$

We call $w$ the Lazard filtration associated with $w$. It induces the natural complete, Hausdorff topology on $k G$, and $\operatorname{gr} k G \cong k\left[X_{1}, \cdots, X_{d}\right]$, where $X_{i}=\operatorname{gr}\left(b_{i}\right)=\operatorname{gr}\left(g_{i}-1\right)$.

Since gr $k G$ is a domain, it follows that $w$ is a valuation.

Now, recall from [13, Definition 1.5.8] the definition of a crossed product, $R * F$, of a ring $R$ with a group $F$. That is $R * F$ is a ring containing $R$, free as an $R$-module with basis $\{\bar{g}: g \in F\} \subseteq R^{\times}$, where $\overline{1_{F}}=1_{R}$, such that $R \overline{g_{1}}=\overline{g_{1}} R$ and $\left(\overline{g_{1}} R\right)\left(\overline{g_{2}} R\right)=\overline{g_{1} g_{2}} R$ for all $g_{1}, g_{2} \in F$.

Also recall from [14] that given a crossed product $S=R * F$, we can define the action $\sigma: F \rightarrow A u t(R)$ and the twist $\gamma: F \times F \rightarrow R^{\times}$such that for all $g, g_{1}, g_{2} \in F, r \in R$ :

$\bar{g} r=\sigma(g)(r) \bar{g}$ and $\overline{g_{1}} \overline{g_{2}}=\gamma\left(g_{1}, g_{2}\right) \overline{g_{1} g_{2}}$.

Proposition 2.2. Let $R$ be a ring with a complete, positive, Zariskian valuation $w: R \rightarrow \mathbb{N} \cup\{\infty\}$, let $F$ be a finite group, and let $S=R * F$ be a crossed product with action $\sigma$ and twist $\gamma$. Suppose that $w(\sigma(g)(r))=w(r)$ for all $g \in F, r \in R$.

Then $w$ extends to a complete, positive, Zariskian filtration $w^{\prime}: S \rightarrow \mathbb{N} \cup\{\infty\}$ defined by $w^{\prime}\left(\sum_{g \in F} r_{g} \bar{g}\right)=$ $\min \left\{w\left(r_{g}\right): g \in G\right\}$, and $g r_{w^{\prime}} S \cong\left(g r_{w} R\right) * F$.

Proof. From the definition it is clear that $w^{\prime}\left(s_{1}+s_{2}\right) \geq \min \left\{w^{\prime}\left(s_{1}\right), w^{\prime}\left(s_{2}\right)\right\}$. So to prove that $w$ defines a ring filtration, it remains to check that $w^{\prime}\left(s_{1} s_{2}\right) \geq w^{\prime}\left(s_{1}\right)+w^{\prime}\left(s_{2}\right)$.

In fact, using the additive property, we only need to prove that $w^{\prime}(r \bar{g} s \bar{h}) \geq w^{\prime}(r \bar{g})+w^{\prime}(s \bar{h})$ for all $r, s \in R$, $g, h \in F$. We will in fact show that equality holds here:

$$
\begin{aligned}
& w^{\prime}(r \bar{g} s \bar{h})=w^{\prime}(r \sigma(g)(s) \bar{g} \bar{h})=w^{\prime}(r \sigma(g)(s) \gamma(g, h) \overline{g h})=w(r \sigma(g)(s) \gamma(g, h)) \\
& =w(r)+w(\sigma(g)(s))+w(\gamma(g, h)) \text { (by the valuation property) } \\
& =w(r)+w(s) .
\end{aligned}
$$

The last equality follows because $w(\sigma(g)(s))=w(s)$ by assumption, and since $R$ is positively filtered and $\gamma(g, h)$ is a unit in $R$, it must have value zero. Clearly $w(r)+w(s)=w^{\prime}(r \bar{g})+w^{\prime}(s \bar{h})$ so we are done.

Hence $w^{\prime}$ is a well-defined ring filtration, clearly $w^{\prime}(r)=w(r)$ for all $r \in R$, and $w^{\prime}(\bar{g})=0$ for all $g \in F$. We can define $\theta: \operatorname{gr}_{w} R \rightarrow \operatorname{gr}_{w^{\prime}} S, r+F_{n+1} R \mapsto r+F_{n+1} S$, which is a well defined, injective ring homomorphism. 
Given $s \in S, s=\sum_{g \in F} r_{g} \bar{g}$, so let $A_{s}:=\left\{g \in F: w\left(r_{g}\right)=w^{\prime}(s)\right\}$. Then:

$$
\operatorname{gr}(s)=\sum_{g \in A_{s}} r_{g} \bar{g}+F_{w^{\prime}(s)+1} S=\sum_{g \in A_{s}}\left(r_{g}+F_{w^{\prime}(s)+1} S\right)\left(\bar{g}+F_{1} S\right)=\sum_{g \in A_{s}} \theta\left(\operatorname{gr}\left(r_{g}\right)\right) \operatorname{gr}(\bar{g}) .
$$

Hence $\operatorname{gr}_{w^{\prime}} S$ is finitely generated over $\theta(\operatorname{gr} R)$ by $\{\operatorname{gr}(\bar{g}): g \in F\}$. This set forms a basis, hence $\operatorname{gr}_{w^{\prime}} S$ is free over $\theta(\operatorname{gr} R)$, and it is clear that each $\operatorname{gr}(\bar{g})$ is a unit in $\operatorname{gr} S$, and they are in bijection with the elements of $F$.

Therefore gr $S$ is Noetherian, and clearly $R * F$ is complete with respect to $w^{\prime}$. Hence $w^{\prime}$ is Zariskian by [12, Chapter II, Theorem 2.1.2].

Finally, $\operatorname{gr}(r \bar{g}) \operatorname{gr}(s \bar{h})=\operatorname{gr}(r \bar{g} s \bar{h})$ since $w^{\prime}(r \bar{g} s \bar{h})=w^{\prime}(r \bar{g})+w^{\prime}(s \bar{h})$, so it is readily checked that $(\operatorname{gr} R) \operatorname{gr}(\bar{g}) \operatorname{gr}(\bar{h})=$ $((\operatorname{gr} R)(\operatorname{gr}(\bar{g}))((\operatorname{gr} R)(\operatorname{gr}(\bar{h}))$, and clearly $(\operatorname{gr} R)(\operatorname{gr}(\bar{g}))=(\operatorname{gr}(\bar{g}))(\operatorname{gr} R)$.

Therefore $\operatorname{gr}_{w^{\prime}} S=\left(\operatorname{gr}_{w} R\right) * F$.

This result will be useful to us later, because for any $p$-valuable group $G, U \unlhd_{o} G, k G \cong k U * \frac{G}{U}$.

\subsection{Mahler expansions}

We will now recap the notion of the Mahler expansion of an automorphism.

Let $G$ be a compact $p$-adic Lie group. Define $C^{\infty}=C^{\infty}(G, k)=\{f: G \rightarrow k: f$ locally constant $\}$, and for each $U \leq_{o} G$, define $C^{\infty U}:=\left\{f \in C^{\infty}: f\right.$ constant on the cosets of $\left.U\right\}$.

Clearly $C^{\infty}$ is a $k$-algebra, $C^{\infty U}$ is a subalgebra, and recall from [2, Lemma 2.9] that there is an action $\gamma: C^{\infty} \rightarrow \operatorname{End}_{k}(k G)$ of $C^{\infty}$ on $k G$. Also recall the following result ([2, Proposition 2.8]):

Proposition 2.3. Let $I$ be a right ideal of $k G, U$ an open subgroup of $k G$. Then $I$ is controlled by $U$ if and only if $I$ is a $C^{\infty U}$-submodule of $k G$, i.e. if and only if for all $g \in G, \gamma\left(\delta_{U g}\right)(I) \subseteq I$, where $\delta_{U g}$ is the characteristic function of the coset $U g$.

Now assume that $G$ is $p$-valuable, with $p$-valuation $\omega$, and let $\underline{g}=\left\{g_{1}, \cdots, g_{d}\right\}$ be an ordered basis for $(G, \omega)$. For each $\alpha \in \mathbb{N}^{d}$ define $i_{\underline{g}}^{(\alpha)}: G \rightarrow k, \underline{g}^{\beta} \mapsto\left(\begin{array}{c}\beta \\ \alpha\end{array}\right)$. Then $i_{\underline{g}}^{(\alpha)} \in C^{\infty}$, so let $\partial_{\underline{g}}^{(\alpha)}:=\gamma\left(i_{\underline{g}}^{(\alpha)}\right)$ - the $\alpha$-quantized divided power with respect to $\underline{g}$.

Also, for each $i=1, \cdots, \bar{d}$, we define $\partial_{i}:=\partial_{\underline{g}}^{\left(e_{i}\right)}$, where $e_{i}$ is the standard $i$ 'th basis vector, these are $k$-linear derivations of $k G$.

Proposition 2.4. Suppose that $I$ is a right ideal of $k G$ and $\partial_{j}(I) \subseteq I$ for some $j \in\{1, \cdots, d\}$. Then $I$ is controlled by a proper open subgroup of $G$.

Proof. Recall from [1, Lemma 7.13] that if $V$ is an open normal subgroup of $G$ with ordered basis $\left\{g_{1}, \cdots, g_{s-1}, g_{s}^{p}, \cdots, g_{r}^{p}, g_{r+1}, \cdots, g_{d}\right\}$ for some $1 \leq s \leq r \leq d$, then for each $g \in G, \gamma\left(\delta_{V g}\right)$ can be expressed as a polynomial in $\partial_{s}, \cdots, \partial_{r}$.

So it follows from Proposition 2.3 that if $\partial_{i}(I) \subseteq I$ for all $i=s, \cdots, r$, then $I$ is controlled by $V$.

Since we know that $\partial_{j}(I) \subseteq I$, it remains to show that we can find a proper, open normal subgroup $U$ of $G$ with ordered basis $\left\{g_{1}, \cdots, g_{j-1}, g_{j}^{p}, g_{j+1}, \cdots, g_{d}\right\}$, and it will follow that $U$ controls $I$.

Notation: Given $d$ variables $x_{1}, \cdots, x_{d}$, we will write $\underline{x}$ to denote the set $\left\{x_{1}, \cdots, x_{d}\right\}, \underline{x}_{j, p}$ to denote the same set, but with $x_{j}$ replaced by $x_{j}^{p}$, and $\underline{x}_{j}$ to denote the set with $x_{j}$ removed altogether. We write $\underline{x}^{\alpha}$ to denote $x_{1}^{\alpha_{1}} \cdots x_{d}^{\alpha_{d}}$.

Let $U$ be the subgroup of $G$ generated topologically by the set $\underline{g}_{j, p}$. It is clear that this subgroup contains $G^{p}$, and hence it is open in $G$. Let us suppose, for contradiction, that it contains an element $u=\underline{g}^{\alpha}$ where $\alpha \in \mathbb{Z}_{p}^{d}$ and $p \nmid \alpha_{j}$.

Recall from [7, Definition 1.8] that the Frattini subgroup $\phi(G)$ of $G$ is defined as the intersection of all maximal open subgroups of $G$, and since $G$ is a pro- $p$ group, it follows from [7, Proposition 1.13] that $\phi(G)$ contains $G^{p}$ and $[G, G]$.

Hence $\phi(G)$ is an open normal subgroup of $G$, and $\frac{G}{\phi(G)}$ is abelian. 
Since $\underline{g}_{j, p}$ generates $U$, it is clear that $\underline{g \phi(G)}_{p, j}$ generates $\frac{U \phi(G)}{\phi(G)}$. Therefore $u \phi(G)=\underline{g \phi(G)_{j, p}^{\beta}}$ for some $\beta \in \mathbb{Z}_{p}^{d}$.

But we know that $u=\underline{g}^{\alpha}$, so $u \phi(G)=\underline{g \phi(G)^{\alpha}}$. So since $\frac{G}{\phi(G)}$ is abelian, it follows that $g_{j}^{\alpha_{j}-p \beta_{j}} \phi(G)=$ $\underline{g \phi(G)}{ }_{j}^{\gamma}$ for some $\gamma \in \mathbb{Z}_{p}^{d}$.

But since $p \nmid \alpha_{j}, \alpha_{j}-p \beta_{j}$ is a $p$-adic unit, and hence $g_{j} \phi(G)=\underline{g \phi(G)^{\delta}}{ }_{j}$, where $\delta_{i}=\gamma_{i}\left(\alpha_{i}-p \beta_{i}\right)^{-1}$. Therefore $\frac{G}{\phi(G)}$ is generated by $\underline{g \phi(G)} j=\frac{\underline{g}_{j} \phi(G)}{\phi(G)}$.

It follows from [7, Proposition 1.9] that $G$ is generated by $\underline{g}_{j}$, which has size $d-1$, and this is a contradiction since the rank $d$ of $G$ is the minimal cardinality of a generating set.

Therefore, every $u \in U$ has the form $\underline{g}_{j, p}^{\beta}$ for some $\beta \in \mathbb{Z}_{p}^{d}$, i.e $\left\{g_{1}, \cdots, g_{j}^{p}, \cdots, g_{d}\right\}$ is an ordered basis for $U$.

Finally, $U$ is maximal, so it contains $\phi(G) \supseteq[G, G]$, and hence it is normal in $G$ as required.

Now, given $\varphi \in \operatorname{Inn}(G)$, clearly $\varphi$ extends to a $k$-linear endomorphism of $k G$, and using Mahler's theorem, we can express $\varphi^{p^{m}}$ as:

$$
\varphi^{p^{m}}=\sum_{\alpha \in \mathbb{N}}\left\langle\varphi^{p^{m}}, \partial_{\underline{g}}^{(\alpha)}\right\rangle \partial_{\underline{g}}^{(\alpha)}=i d+c_{1, m} \partial_{1}+\ldots+c_{d, m} \partial_{d}+\ldots
$$

Where $\left\langle\varphi^{p^{m}}, \partial_{\underline{g}}^{(\alpha)}\right\rangle \in k G$ is the $\alpha$-Mahler coefficient of $\varphi^{p^{m}}$ (see [1, Corollary 6.6] for full details).

Given $P \in \operatorname{Spec}^{f}(k G)$, our general approach is to choose $\varphi \neq i d$, and use analysis of (2) to obtain a sequence of endomorphisms preserving $P$, converging pointwise in $m$ to an expression involving only $\partial_{1}, \cdots, \partial_{d}$, which will imply a control theorem using Proposition 2.4

However, it is more convenient for us to reduce modulo $P$, whence we can pass to the ring of quotients $Q\left(\frac{k G}{P}\right)$ and divide out by anything regular $\bmod P$.

So let $\tau: k G \rightarrow Q\left(\frac{k G}{P}\right)$ be the natural map, then inside $\operatorname{End}_{k}\left(k G, Q\left(\frac{k G}{P}\right)\right)$ our expression becomes:

$$
\tau \varphi^{p^{m}}-\tau=\sum_{\alpha \in \mathbb{N}} \tau\left(\left\langle\varphi^{p^{m}}, \partial_{\underline{g}}^{(\alpha)}\right\rangle\right) \tau \partial_{\underline{g}}^{(\alpha)}
$$

Since we want to analyse convergence of this expression as $m \rightarrow \infty$, we need to define a certain well behaved filtration $v$ on $Q\left(\frac{k G}{P}\right)$, which we call a non-commutative valuation. We will describe the construction of $v$ in the next section.

In our case, we take $G$ to be non-abelian, $p$-valuable, abelian-by-procyclic group, with principal subgroup $H$, procyclic element $X$, and we take $\varphi$ to be conjugation by $X$, which is a non-trivial inner automorphism.

It is clear that $\left.\varphi\right|_{H}$ is trivial modulo centre since $H$ is abelian. Therefore it follows from the proof of [1, Lemma 6.7] that if $g=\left\{h_{1}, \cdots, h_{d}, X\right\}$ is an ordered basis for $(G, \omega)$, where $\left\{h_{1}, \cdots, h_{d}\right\}$ is some ordered basis for $H$, then for any $m \in \mathbb{N} \alpha \in \mathbb{N}^{d+1}$, we have:

$$
\left\langle\varphi^{p^{m}}, \partial_{\underline{g}}^{(\alpha)}\right\rangle=\left(\varphi^{p^{m}}\left(h_{1}\right) h_{1}^{-1}-1\right)^{\alpha_{1}} \cdots\left(\varphi^{p^{m}}\left(h_{d}\right) h_{d}^{-1}-1\right)^{\alpha_{d}}\left(\varphi^{p^{m}}(X) X^{-1}-1\right)^{\alpha_{d+1}}
$$

And since $\varphi(X)=X$, it is clear that this is 0 if $\alpha_{d+1} \neq 0$. Hence we may assume that $\alpha \in \mathbb{N}^{d}$, and:

$$
\left\langle\varphi^{p^{m}}, \partial_{\underline{g}}^{(\alpha)}\right\rangle=\left(\varphi^{p^{m}}\left(h_{1}\right) h_{1}^{-1}-1\right)^{\alpha_{1}} \cdots\left(\varphi^{p^{m}}\left(h_{d}\right) h_{d}^{-1}-1\right)^{\alpha_{d}}
$$

Recall our function $u: H \rightarrow H$ defined in section 2.1, we can now use $u$ to approximate the Mahler coefficients inside $Q\left(\frac{k G}{P}\right)$. Setting $q_{i}=\tau\left(u\left(h_{i}\right)-1\right)$ for $i=1, \ldots, d$, it follows from the proof of [1, Proposition 7.7] that we can derive the following expression from (3):

$$
\tau \varphi^{p^{m}}-\tau=q_{1}^{p^{m}} \tau \partial_{1}+\ldots .+q_{d}^{p^{m}} \tau \partial_{d}+\sum_{|\alpha| \geq 2} \underline{q}^{\alpha p^{m}} \tau \partial_{\underline{g}}^{(\alpha)}+\varepsilon_{m}
$$

Where $\underline{q}^{\alpha p^{m}}=q_{1}^{\alpha_{1}} \cdots q_{d}^{\alpha_{d}}, \varepsilon_{m}=\sum_{\alpha \in \mathbb{N}^{d}}\left(\left\langle\varphi^{p^{m}}, \partial_{\underline{g}}^{(\alpha)}\right\rangle-\underline{q}^{\alpha p^{m}}\right) \tau \partial_{\underline{g}}^{(\alpha)} \in \operatorname{End}_{k}(k G, Q)$, and there exists $t \in \mathbb{N}$ such that $v\left(\varepsilon_{m}(r)\right)>p^{2 m-t}$ for all $r \in k G$. 
Since $\varphi(P)=P$ it is clear that the left hand side of this expression annihilates $P$. So take any $y \in P$ and apply it to both sides of (4) and we obtain:

$$
0=q_{1}^{p^{m}} \tau \partial_{1}(y)+\ldots+q_{d}^{p^{m}} \tau \partial_{d}(y)+O\left(q^{p^{m}}\right)
$$

Where $q \in Q$ with $v\left(q^{p^{m}}\right) \geq \min _{i \leq d}\left\{2 v\left(q_{i}^{p^{m}}\right)\right\}$ for all $m$.

Furthermore, let $f(x)=a_{0} x+a_{1} x^{p}+a_{2} x^{p^{2}}+\cdots+a_{n} x^{p^{n}}$ be a polynomial, where $a_{i} \in \tau(k H)$ for each $i$.

Then for each $m \in \mathbb{N}, i=0, \cdots, n$, consider expression (5) above, with $m$ replaced by $m+i$, and multiply by $a_{i}^{p^{m}}$ to obtain:

$$
0=\left(a_{i} q_{1}^{p^{i}}\right)^{p^{m}} \tau \partial_{1}(y)+\ldots+\left(a_{i} q_{d}^{p^{i}}\right)^{p^{m}} \tau \partial_{d}(y)+O\left(\left(a_{i} q^{p^{i}}\right)^{p^{m}}\right)
$$

Sum all these expressions as $i$ ranges from 0 to $n$ to obtain:

$$
0=f\left(q_{1}\right)^{p^{m}} \tau \partial_{1}(y)+\ldots+f\left(q_{d}\right)^{p^{m}} \tau \partial_{d}(y)+O\left(f(q)^{p^{m}}\right)
$$

In the next section, we will see how after ensuring certain conditions on $f$, we can use this expression to deduce a control theorem.

\section{Non-commutative Valuations}

In this section, we fix $Q$ a simple Artinian ring. First, recall from [1] the definition of a non-commutative valuation.

Definition 3.1. A non-commutative valuation on $Q$ is a Zariskian filtration $v: Q \rightarrow \mathbb{Z} \cup\{\infty\}$ such that if $\widehat{Q}$ is the completion of $Q$ with respect to $v$, then $\widehat{Q} \cong M_{k}(Q(D))$ for some complete non-commutative $D V R$, and $v$ is induced by the natural $J(D)$-adic filtration.

It follows from this definition that if $q \in Q$ with $v(q) \geq 0$, then $q$ is $v$-regular if and only if $q$ is normal in $F_{0} \widehat{Q}$.

We want to construct a non-commutative valuation $v$ on $Q\left(\frac{k G}{P}\right)$ which we can use to analyse our Mahler expansion (6).

\subsection{Construction}

Recall that [1, Theorem $\mathrm{C}$ ] gives that if $R$ is a prime ring carrying a Zariskian filtration such that gr $R$ is commutative, then we can construct a non-commutative valuation on $Q(R)$.

The main theorem of this section generalises this result.

Let $R$ be a prime ring with a positive Zariskian filtration $w: R \rightarrow \mathbb{N} \cup\{\infty\}$ such that $\operatorname{gr}_{w} R$ is finitely generated over a central, graded, Noetherian subring $A$, and we will assume that the positive part $A_{>0}$ of $A$ is not nilpotent, and hence we may fix a minimal prime ideal $\mathfrak{q}$ of $A$ with $\mathfrak{q} \nsupseteq A_{>0}$. Define:

$$
T=\{X \in A \backslash \mathfrak{q}: X \text { is homogeneous }\} .
$$

Then $T$ is central, and hence localisable in gr $R$, and the left and right localisations agree.

Lemma 3.1. Let $\mathfrak{q}^{\prime}:=T^{-1} \mathfrak{q}$, then $\mathfrak{q}^{\prime}$ is a nilpotent ideal of $T^{-1} A$ and:

i. There exists $Z \in T$, homogeneous of positive degree, such that $\frac{T^{-1} A}{\mathfrak{q}^{\prime}} \cong\left(\frac{T^{-1} A}{\mathfrak{q}^{\prime}}\right)_{0}\left[\bar{Z}, \bar{Z}^{-1}\right]$, where $\bar{Z}:=Z+\mathfrak{q}$.

ii. The quotient $\frac{\left(T^{-1} A\right)_{>0}}{Z\left(T^{-1} A\right)_{\geq 0}}$ is Artinian, and $T^{-1} A$ is gr-Artinian, i.e. every descending chain of graded ideals terminates.

Proof. Since $A$ is a graded, commutative, Noetherian ring, this is identical to the proof of [1, Proposition $3.2]$. 
Since gr $R$ is finitely generated over $A$, it follows that $T^{-1} \operatorname{gr} R$ is finitely generated over $T^{-1} A$. So using this lemma, we see that $T^{-1}$ gr $R$ is gr-Artinian.

Let $S:=\{r \in R: \operatorname{gr}(r) \in T\}$, then since $w$ is Zariskian, $S$ is localisable by [11, Corollary 2.2], and $S^{-1} R$ carries a Zariskian filtration $w^{\prime}$ such that $\operatorname{gr}_{w^{\prime}} S^{-1} R \cong T^{-1}$ gr $R$, and if $r \in R$ then $w^{\prime}(r) \geq w(r)$, and equality holds if $r \in S$.

Furthermore, $w^{\prime}$ satisfies $w^{\prime}\left(s^{-1} r\right)=w^{\prime}(r)-w(s)$ for all $r \in \operatorname{gr} R, s \in S$.

Now, since $R$ is prime, the proof of [1, Lemma 3.3] shows that $S^{-1} R=Q(R)$, so let $Q^{\prime}$ be the completion of $Q(R)$ with respect to $w^{\prime}$.

Let $U:=F_{0} Q^{\prime}$, which is Noetherian by [12, Ch.II Lemma 2.1.4] then $\operatorname{gr}_{w^{\prime}} U \cong\left(T^{-1} \operatorname{gr} R\right)_{\geq 0}$, and since gr $Q^{\prime}=T^{-1}$ gr $R$ is gr-Artinian, $Q^{\prime}$ is Artinian.

Lemma 3.2. There exists a regular, normal element $z \in J(U) \cap Q^{\prime \times}$ such that $\frac{U}{z U}$ has Krull dimension 1 on both sides, and for all $n \in \mathbb{Z}, F_{n w^{\prime}(z)} Q^{\prime}=z^{n} U$, hence the $z$-adic filtration on $Q^{\prime}$ is topologically equivalent to $w^{\prime}$.

Proof. Recall the element $Z \in T^{-1} A$ from Lemma $3.1(i)$, then we can choose an element $z \in U$ such that $\operatorname{gr}_{w^{\prime}}(z)=Z$. Since $Z$ is a unit in $T^{-1} A$, we can in fact choose $z$ to be regular and normal in $U$. Then since $w^{\prime}$ is Zariskian and $Z$ has positive degree, $z \in F_{1} Q^{\prime} \subseteq J(U)$.

Furthermore, since $\bar{Z}=Z+\mathfrak{q}^{\prime}$ is a unit in $\frac{T^{-1} A}{\mathfrak{q}^{\prime}}$ and $\mathfrak{q}^{\prime}$ is nilpotent, it follows that $Z$ is a unit in $T^{-1} A$, and hence in $T^{-1} \operatorname{gr} R=\operatorname{gr} Q^{\prime}$.

So it follows that $z$ is not a zero divisor in $Q^{\prime}$, and hence is a unit since $Q^{\prime}$ is artinian. Also, for all $u \in Q^{\prime}$, $w^{\prime}\left(z u z^{-1}\right)=w^{\prime}(u)$ since $Z$ is central in gr $Q^{\prime}$, hence $z$ is normal in $U$.

Since $\left(T^{-1} \operatorname{gr} R\right)_{\geq 0}$ is finitely generated over $\left(T^{-1} A\right)_{\geq 0}$, it follows that $\frac{\operatorname{gr} U}{\operatorname{Zgr} U}$ is finitely generated over the image of $\frac{\left(T^{-1} A\right)_{\geq 0}}{Z\left(T^{-1} A\right)_{\geq 0}} \rightarrow \frac{\operatorname{gr} U}{Z \operatorname{gr} U}$.

This image is gr-Artinian by Lemma $3.1(i i)$ and hence $\frac{\operatorname{gr} U}{Z \operatorname{gr} U}$ it is also gr-Artinian.

Therefore $\frac{U}{z U}$ is Artinian, and the proof of [1, Proposition 3.4] gives us that $U$ has Krull dimension at most 1 on both sides, and that $F_{n w^{\prime}(z)} Q^{\prime}=z^{n} U$ for all $n \in \mathbb{Z}$.

So, after passing to a simple quotient $\widehat{Q}$ of $Q^{\prime}$, and letting $V:=\widehat{Q}_{\geq 0}$ be the image of $U$ in $\widehat{Q}$, then since $Q(R)$ is simple, it follows that the map $Q(R) \rightarrow \widehat{Q}$ is injective, and the image is dense with respect to the quotient filtration.

Now, choose a maximal order $\mathcal{O}$ in $\widehat{Q}$, which is equivalent to $V$ in the sense of [13, Definition 1.9]. Such an order exists by [1, Theorem 3.11], and it is Noetherian.

Furthermore, let $z \in J(U)$ be the regular, normal element from Lemma 3.2, and let $\bar{z} \in J(V)$ be the image of $z$ in $V$, then $\mathcal{O} \subseteq \bar{z}^{-r} V$ for some $r \in \mathbb{N}$ by [1, Proposition 3.7].

It follows from [1, Theorem 3.6] that $\mathcal{O} \cong M_{n}(D)$ for some complete non-commutative DVR $D$, and hence $\widehat{Q} \cong M_{n}(Q(D))$. So let $v$ be the $J(\mathcal{O})$-adic filtration, i.e. the filtration induced from the valuation on $D$. Then $v$ is topologically equivalent to the $\bar{z}$-adic filtration on $\widehat{Q}$.

It is clear from the definition that the restriction of $v$ to $Q(R)$ is a non-commutative valuation, and the proof of [1, Theorem $\mathrm{C}$ ] shows that $(R, w) \rightarrow(Q(R), v)$ is continuous.

Note that our construction depends on a choice of minimal prime ideal $\mathfrak{q}$ of $A$. So altogether, we have proved the following:

Theorem 3.3. Let $R$ be a prime ring with a Zariskian filtration $w: R \rightarrow \mathbb{N} \cup\{\infty\}$ such that gr $R$ is finitely generated over a central, graded, Noetherian subring $A$, and the positive part $A_{>0}$ of $A$ is not nilpotent.

Then for every minimal prime ideal $\mathfrak{q}$ of $A$ with $q \nsupseteq A_{>0}$, there exists a corresponding non-commutative valuation $v_{\mathfrak{q}}$ on $Q(R)$ such that the inclusion $(R, w) \rightarrow\left(Q(R), v_{\mathfrak{q}}\right)$ is continuous. 
In particular, if $P$ is a prime ideal of $k G$, then $R=\frac{k G}{P}$ carries a natural Zariskian filtration, given by the quotient of the Lazard filtration on $k G$, and $\operatorname{gr} R \cong \frac{\operatorname{gr} k G}{\operatorname{gr} P}$ is commutative, and if $P \neq J(k G)$ then $(\operatorname{gr} R)_{\geq 0}$ is not nilpotent by [1, Lemma 7.2].

Hence we may apply Theorem 3.3 to obtain a non-commutative valuation $v$ on $Q\left(\frac{k G}{P}\right)$ such that the natural $\operatorname{map} \tau:(k G, w) \rightarrow\left(Q\left(\frac{k G}{P}\right), v\right)$ is continuous.

\subsection{Properties}

We will now explore some important properties of the non-commutative valuation $v_{\mathfrak{q}}$ on $Q(R)$ that we have constructed.

So again, we have that gr $R$ is finitely generated over $A$, and $\mathfrak{q}$ is a minimal prime ideal of $A$, not containing $A_{>0}$. Recall first the data that we used in the construction of $v_{\mathfrak{q}}$ :

- $w^{\prime}$ - a Zariskian filtration on $Q(R)$ such that $w^{\prime}(r) \geq w(r)$ for all $r \in R$, with equality if $\operatorname{gr}_{w}(r) \in A \backslash \mathfrak{q}$. Moreover, if $\operatorname{gr}_{w}(r) \in A \backslash \mathfrak{q}$ then $r$ is $w^{\prime}$-regular.

- $Q^{\prime}$ - the completion of $Q(R)$ with respect to $w^{\prime}$.

- $U$ - the positive part of $Q^{\prime}$, a Noetherian ring.

- $z$ - a regular, normal element of $J(U)$ such that $z^{n} U=F_{n w^{\prime}(z)} Q^{\prime}$ for all $n \in \mathbb{Z}$.

- $v_{z, U}$ - the $z$-adic filtration on $Q^{\prime}$, topologically equivalent to $w^{\prime}$.

- $\widehat{Q}$ - a simple quotient of $Q^{\prime}$.

- $V$ - the positive part of $\widehat{Q}$, which is the image of $U$ in $\widehat{Q}$.

- $\bar{z}$ - the image of $z$ in $V$.

- $v_{\bar{z}, V}$ - the $\bar{z}$-adic filtration on $\widehat{Q}$, topologically equivalent to the quotient filtration.

- $\mathcal{O}$ - a maximal order in $\widehat{Q}$, equivalent to $V$, satisfying $\mathcal{O} \subseteq \bar{z}^{-r} V$ for some $r \geq 0$.

- $v_{\bar{z}, \mathcal{O}}$ - the $\bar{z}$-adic filtration on $\mathcal{O}$.

- $v_{\mathfrak{q}}$ - the $J(\mathcal{O})$-adic filtration on $\widehat{Q}$, topologically equivalent to $v_{\bar{z}, \mathcal{O}}$.

From now on, we will assume further that $R$ is an $\mathbb{F}_{p}$-algebra.

Lemma 3.4. Given $r \in R$ such that $g r(r) \in A \backslash \mathfrak{q}$, we have:

i. $r$ is normal in $U$, a unit in $Q^{\prime}$ and for any $u \in U, w^{\prime}\left(r u r^{-1}-u\right)>w^{\prime}(u)$.

ii $v_{\bar{z}, V}(r)=v_{z, U}(r)$.

Proof. $i$. Since $r \in S=\{s \in R: \operatorname{gr}(s) \in A \backslash \mathfrak{q}\}$ and $Q(R)=S^{-1} R, r$ is a unit in $Q^{\prime}$, and we know that $w^{\prime}(r)=w(r)$. Given $u \in U$, we want to prove that $r u r^{-1} \in U$, thus showing that $r$ is normal in $U$.

We know that $U=F_{0} Q^{\prime}$ is the completion of the positive part $F_{0} Q(R)$ of $Q(R)$ by definition, and we may assume that $u$ lies in $Q(R)$, i.e. $u=s^{-1} t$ for some $s \in S, t \in R$, and $w^{\prime}(u)=w^{\prime}(t)-w(s) \geq 0$.

But $\operatorname{gr}(r), \operatorname{gr}(s) \notin \mathfrak{q}$, and hence $\operatorname{gr}(r) \operatorname{gr}(s) \neq 0$, which means that $w(r s)=w(r)+w(s)$. Therefore $w^{\prime}\left(r^{-1} u r\right)=w^{\prime}\left((s r)^{-1} t r\right)=w^{\prime}(t r)-w(r s) \geq w^{\prime}(t)+w^{\prime}(r)-w(r)-w(s)=w^{\prime}(t)-w^{\prime}(s) \geq 0$, and so $r^{-1} u r \in U$ as required.

Furthermore, since $\operatorname{gr}(r) \in A$ is central in $g r R, w^{\prime}(r u-u r)>w^{\prime}(u)+w^{\prime}(r)$, and thus $w^{\prime}\left(r u r^{-1}-u\right)=$ $w^{\prime}\left((r u-u r) r^{-1}\right) \geq w^{\prime}(r u-u r)-w^{\prime}(r)>w^{\prime}(u)+w^{\prime}(r)-w^{\prime}(r)=w^{\prime}(u)$.

ii. Let $t:=v_{z, U}(r)$.

So $r \in z^{t} U \backslash z^{t+1} U=F_{t w^{\prime}(z)} Q^{\prime} \backslash F_{(t+1) w^{\prime}(z)} Q^{\prime}$, and hence $w^{\prime}(r)=t w^{\prime}(z)+j$ for some $0 \leq j<w^{\prime}(z)$.

Since $\operatorname{gr}_{w^{\prime}}(r) \in A \backslash \mathfrak{q}$, we have that $w^{\prime}\left(r^{-1}\right)=-w^{\prime}(r)=-t w^{\prime}(z)-j$. 
Let $\bar{r}$ be the image of $r$ in $\widehat{Q}$. Then since $r \in z^{t} U$, it is clear that $\bar{r} \in \bar{z}^{t} V$, hence $v_{\bar{z}, V}(r) \geq t$, so it remains to prove that $v_{\bar{z}, V}(r) \leq t$.

Suppose that $\bar{r} \in \bar{z}^{t+1} V$, i.e. $r-z^{t+1} u$ maps to zero in $\widehat{Q}$ for some $u \in U$, and hence $z^{-t} r-z u=z^{-t}\left(r-z^{t+1} u\right)$ also maps to zero.

Let $a=z^{-t} r, b=-z u$. Then $w^{\prime}(b) \geq w^{\prime}(u)+w^{\prime}(z) \geq w^{\prime}(z), w^{\prime}\left(a^{-1}\right)=w^{\prime}\left(r^{-1}\right)+t w^{\prime}(z)=-t w^{\prime}(z)-j+$ $t w^{\prime}(z)=-j$, so $w^{\prime}\left(a^{-1} b\right) \geq w^{\prime}(z)-j>w^{\prime}(z)-w^{\prime}(z)=0$, and therefore $\left(a^{-1} b\right)^{n} \rightarrow 0$ as $n \rightarrow \infty$.

So by completeness of $Q^{\prime}$, the series $\sum_{n \geq 0}(-1)^{n}\left(a^{-1} b\right)^{n} a^{-1}$ converges in $Q^{\prime}$, and the limit is the inverse of $a+b$, hence $a+b=z^{-t} r-z u$ is a unit in $\bar{Q}^{\prime}$.

Therefore a unit in $Q^{\prime}$ maps to zero in $\widehat{Q}$ - contradiction.

Hence $\bar{r} \notin \bar{z}^{t+1} V$, so $v_{\bar{z}, V}(r) \leq t$ as required.

Proposition 3.5. Let $u \in U$ be regular and normal, then $u$ is a unit in $Q^{\prime}$. Furthermore, if $w^{\prime}\left(u a u^{-1}-a\right)>$ $w^{\prime}(a)$ for all $a \in Q^{\prime}$, then setting $\bar{u}$ as the image of $u$ in $V$, we have that for sufficiently high $m \in \mathbb{N}, \bar{u}^{p^{m}}$ is $v_{\mathfrak{q}}$-regular.

Proof. Since $u$ is regular in $U$, it is not a zero divisor, so it follows that $u$ is not a zero divisor in $Q^{\prime}$, and hence a unit since $Q^{\prime}$ is artinian.

Since $u$ is normal in $U$, i.e. $u U=U u$, it follows that $\bar{u} V=V \bar{u}$, so $\bar{u}$ is normal in $V$. We want to prove that for $m$ sufficiently high, $\bar{u}^{p^{m}}$ is normal in $\mathcal{O}=F_{0} \widehat{Q}$, and it will follow that it is $v_{\mathfrak{q}}$-regular.

We know that $w^{\prime}\left(u a u^{-1}-a\right)>w^{\prime}(a)$ for all $a \in Q^{\prime}$, so let $\theta: Q^{\prime} \rightarrow Q^{\prime}$ be the conjugation action of $u$, then $(\theta-i d)\left(F_{n} Q^{\prime}\right) \subseteq F_{n+1} Q^{\prime}$ for all $n \in \mathbb{Z}$.

Therefore, for all $k \in \mathbb{N},(\theta-i d)^{k}\left(F_{n} Q^{\prime}\right) \subseteq F_{n+k} Q^{\prime}$.

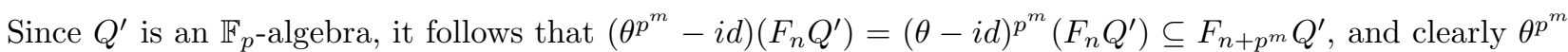
is conjugation by $\bar{u}^{p^{m}}$.

So fix $k \in \mathbb{N}$ such that $p^{k} \geq w^{\prime}(z)$. Then we know that $z^{n} U=F_{n w^{\prime}(z)} U$, so $\left(\theta^{p^{k}}-i d\right)\left(z^{n} U\right) \subseteq F_{n w^{\prime}(z)+p^{k}} U \subseteq$ $F_{(n+1) w^{\prime}(z)} U=z^{n+1} U$.

Hence we have that for all $a \in Q^{\prime}, v_{z, U}\left(u^{p^{k}} a u^{-p^{k}}-a\right)>v_{z, U}(a)$, and it follows immediately that $v_{\bar{z}, V}\left(\bar{u}^{p^{k}} a \bar{u}^{-p^{k}}-\right.$ a) $>v_{\bar{z}, V}(a)$ for all $a \in \widehat{Q}$.

For convenience, let $v:=\bar{u}^{p^{k}} \in V$. We know that $v_{\bar{z}, V}\left(v a v^{-1}-a\right)>v_{\bar{z}, V}(a)$ for all $a \in \widehat{Q}$, and we want to prove that $v^{p^{m}}$ is normal in $\mathcal{O}$ for $m$ sufficently high.

Let $I=\{v \in V: q v \in V$ for all $q \in \mathcal{O}\}$, then $I$ is a two-sided ideal of $V$, and since $\mathcal{O} \subseteq \bar{z}^{-r} V$, we have that $\bar{z}^{r} V \subseteq I$.

Let $\psi: \widehat{Q} \rightarrow \widehat{Q}$ be conjugation by $v$, then we know that $(\psi-i d)\left(\bar{z}^{n} V\right) \subseteq \bar{z}^{n+1} V$, and hence $(\psi-i d)^{s}(V) \subseteq \bar{z}^{s} V$ for all $s$.

Choose $m \in \mathbb{N}$ such that $p^{m} \geq r$, then $\left(\psi^{p^{m}}-i d\right)(V)=(\psi-i d)^{p^{m}}(V) \subseteq \bar{z}^{p^{m}} V \subseteq \bar{z}^{r} V \subseteq I$.

Therefore, for all $a \in V, v^{p^{m}} a v^{-p^{m}}-a \in I$, and in particular, for all $a \in I, v^{p^{m}} a v^{-p^{m}} \in I$, so $v^{p^{m}} I v^{-p^{m}} \subseteq I$.

So set $b:=v^{p^{m}}$, then it follows from Noetherianity of $V$ that $b I b^{-1}=I$.

Finally, consider the subring $\mathcal{O}^{\prime}:=b^{-1} \mathcal{O} b$ of $\widehat{Q}$ containing $V$, then since $\mathcal{O}$ is a maximal order equivalent to $V$, it follows immediately that $\mathcal{O}^{\prime}$ is equivalent to $V$, and that $\mathcal{O}^{\prime}$ is also maximal.

Given $c \in \mathcal{O}^{\prime}, c=b^{-1} q b$ for some $q \in \mathcal{O}$. So given $x \in I, c x=b^{-1} q b x=b^{-1} q b x b^{-1} b \in b^{-1} I b=I$, so $c \in \mathcal{O}_{l}(I)=\{q \in \widehat{Q}: q I \subseteq I\}$, and hence $\mathcal{O}^{\prime} \subseteq \mathcal{O}_{l}(I)$.

But $\mathcal{O}_{l}(I)$ is a maximal order in $\widehat{Q}$, equivalent to $V$ by [13, Lemma 1.12], and this order contains $\mathcal{O}$ by the definition of $I$. 

$b \mathcal{O} b^{-1}$.

So since $\mathcal{O}$ and $\mathcal{O}^{\prime}$ are maximal orders and are both contained in $\mathcal{O}_{l}(I)$, it follows that $\mathcal{O}_{l}(I)=\mathcal{O}=\mathcal{O}^{\prime}=$

Therefore $b=v^{p^{m}}=\bar{u}^{p^{m+k}}$ is normal in $\mathcal{O}$ as required.

In particular, it is clear that $z \in U$ satisfies the property that $w^{\prime}\left(z a z^{-1}-a\right)>w^{\prime}(a)$ for all $a \in Q^{\prime}$, thus $\bar{z}^{p^{m}}$ is normal in $\mathcal{O}$ for large $m$.

The next result will be very useful to us later when we want to compare values of elements in $Q\left(\frac{k G}{P}\right)$ based on their values in $k G$.

Theorem 3.6. Given $r \in R$ such that $g r_{w}(r) \in A \backslash \mathfrak{q}$, there exists $m \in \mathbb{N}$ such that $r^{p^{m}}$ is $v_{\mathfrak{q}}$-regular inside $\widehat{Q}$. Also, if $s \in R$ with $w(s)>w(r)$ then for sufficiently high $m, v_{\mathfrak{q}}\left(s^{p^{m}}\right)>v_{\mathfrak{q}}\left(r^{p^{m}}\right)$.

Moreover, if $w(s)=w(r)$ and $g r_{w}(s) \in \mathfrak{q}$ then we also have that $v_{\mathfrak{q}}\left(s^{p^{m}}\right)>v_{\mathfrak{q}}\left(r^{p^{m}}\right)$ for sufficiently high $m$.

Proof. Since $\operatorname{gr}_{w}(r) \in A \backslash \mathfrak{q}$, it follows from Lemma 3.4 $(i)$ that $r$ is normal and regular in $U$, and $w^{\prime}\left(r u r^{-1}-u\right)>$ $w^{\prime}(u)$ for all $u \in U$. So for $m \in \mathbb{N}$ sufficiently high, $r^{p^{m}}$ is $v_{\mathfrak{q}}$-regular by Proposition 3.5.

Note that since $\operatorname{gr}_{w}(r) \in A \backslash \mathfrak{q}$, we have that $w^{\prime}(r)=w(r)$. In fact, since $\operatorname{gr}_{w}(r)$ is not nilpotent, we actually have that $w^{\prime}\left(r^{n}\right)=w\left(r^{n}\right)=n w(r)$ for all $n \in \mathbb{N}$. So if $w(s)>w(r)$, then for any $n, w^{\prime}\left(s^{n}\right) \geq n w(s)>$ $n w(r)=w^{\prime}\left(r^{n}\right)$.

Moreover, if $w(s)=w(r)$ and $\operatorname{gr}_{w}(s) \in \mathfrak{q}$, then since $\mathfrak{q}^{\prime}=T^{-1} \mathfrak{q}$ is nilpotent by Lemma 3.1 it follows that for $n$ sufficiently high, $w^{\prime}\left(s^{n}\right)>n w^{\prime}(s)$, and hence $w^{\prime}\left(s^{n}\right)>n w(s)=n w(r)=w\left(r^{n}\right)=w^{\prime}\left(r^{n}\right)$.

So, in either case, after replacing $r$ and $s$ by high $p^{\prime}$ th powers of $r$ and $s$ if necessary, we may assume that $w^{\prime}(s)>w^{\prime}(r)$, i.e. $w^{\prime}(s) \geq w^{\prime}(r)+1$.

It follows that for every $K>0$, we can find $m \in \mathbb{N}$ such that $w^{\prime}\left(s^{p^{m}}\right) \geq w^{\prime}\left(r^{p^{m}}\right)+K$. First we will prove the same result for $v_{z, U}$ :

Given $K>0$, let $N=w^{\prime}(z)(K+1)$, so that $K=\frac{1}{w^{\prime}(z)} N-1$, then choose $m$ such that $w^{\prime}\left(s^{p^{m}}\right) \geq w^{\prime}\left(r^{p^{m}}\right)+N$, and let $l:=v_{z, U}\left(s^{p^{m}}\right), t:=v_{z, U}\left(r^{p^{m}}\right)$.

So $s^{p^{m}} \in z^{l} U \backslash z^{l+1} U=F_{l w^{\prime}(z)} Q^{\prime} \backslash F_{(l+1) w^{\prime}(z)} Q^{\prime}$, and $r^{p^{m}} \in z^{t} U \backslash z^{t+1} U=F_{l w^{\prime}(z)} Q^{\prime} \backslash F_{(l+1) w^{\prime}(z)} Q^{\prime}$.

Hence $(l+1) w^{\prime}(z) \geq w^{\prime}\left(s^{p^{m}}\right) \geq l w^{\prime}(z)$ and $(t+1) w^{\prime}(z) \geq w^{\prime}\left(r^{p^{m}}\right) \geq t w^{\prime}(z)$.

Therefore, $v_{z, U}\left(s^{p^{m}}\right)=l=\frac{1}{w^{\prime}(z)}\left((l+1) w^{\prime}(z)\right)-1 \geq \frac{1}{w^{\prime}(z)} w^{\prime}\left(s^{p^{m}}\right)-1$

$\geq \frac{1}{w^{\prime}(z)}\left(w^{\prime}\left(r^{p^{m}}\right)+N\right)-1 \geq \frac{1}{w^{\prime}(z)}\left(t w^{\prime}(z)+N\right)-1=t+\frac{1}{w^{\prime}(z)} N-1=t+K=v_{z, U}\left(r^{p^{m}}\right)+K$ as required.

Now, since $\operatorname{gr}_{w}(r) \in A \backslash \mathfrak{q}$, we have that $v_{\bar{z}, V}\left(r^{p^{m}}\right)=v_{z, U}\left(r^{p^{m}}\right)$ for all $m$ by Lemma $3.4(i i)$.

Therefore, since $v_{\bar{z}, V}\left(s^{p^{m}}\right) \geq v_{z, U}\left(s^{p^{m}}\right)$ for all $m$, it follows that for every $K>0$, there exists $m \in \mathbb{N}$ such that $v_{\bar{z}, V}\left(s^{p^{m}}\right) \geq v_{\bar{z}, V}\left(r^{p^{m}}\right)+K$.

Now we will consider $v_{\bar{z}, \mathcal{O}}$, the $\bar{z}$-adic filtration on $\widehat{Q}$.

Recall that $V \subseteq \mathcal{O} \subseteq \bar{z}^{-r} V$, and thus $z^{n} V \subseteq z^{n} \mathcal{O} \subseteq z^{n-r} V$ for all $n$. Hence $v_{\bar{z}, V}(v)-r \leq v_{\bar{z}, \mathcal{O}}(v) \leq v_{\bar{z}, V}(v)$ for all $v \in V$.

For any $K>0$, choose $m$ such that $v_{\bar{z}, V}\left(s^{p^{m}}\right) \geq v_{\bar{z}, V}\left(r^{p^{m}}\right)+K+r$. Then:

$$
v_{\bar{z}, \mathcal{O}}\left(s^{p^{m}}\right) \geq v_{\bar{z}, V}\left(s^{p^{m}}\right)-r \geq v_{\bar{z}, V}\left(r^{p^{m}}\right)+K+r-r \geq v_{\bar{z}, \mathcal{O}}\left(r^{p^{m}}\right)+K .
$$

Now, using Proposition 3.5, we know that we can find $k \in \mathbb{N}$ such that $x:=\bar{z}^{p^{k}}$ is normal in $\mathcal{O}$, i.e. $x \mathcal{O}=\mathcal{O} x$ is a two-sided ideal of $\mathcal{O}$.

Then since $\mathcal{O} \cong M_{n}(D)$ for some non-commutative DVR $D$, it follows that $x \mathcal{O}=J(\mathcal{O})^{a}$ for some $a \in \mathbb{N}$, and $x^{m} \mathcal{O}=J(\mathcal{O})^{a m}$ for all $m$. 
So, choose $m \in \mathbb{N}$ such that $r^{p^{m}}$ is $v_{\mathfrak{q}}$-regular, $v_{\mathfrak{q}}\left(r^{p^{m}}\right) \geq a$ and $v_{\bar{z}, \mathcal{O}}\left(s^{p^{m}}\right) \geq v_{\bar{z}, \mathcal{O}}\left(r^{p^{m}}\right)+p^{k}$.

Then suppose that $v_{\mathfrak{q}}\left(r^{p^{m}}\right)=n$, i.e. $r^{p^{m}} \in J(\mathcal{O})^{n} \backslash J(\mathcal{O})^{n+1}$ and $n \geq a$.

We have that $n=q a+t$ for some $q, t \in \mathbb{N}, 0 \leq t<a$, so $q \geq 1$ and $q a \leq n<n+1 \leq(q+1) a$. Therefore:

$$
r^{p^{m}} \in J(\mathcal{O})^{n} \subseteq J(\mathcal{O})^{q a}=x^{q} \mathcal{O}=z^{p^{k} q} \mathcal{O} \text {, and so } v_{\bar{z}, \mathcal{O}}\left(r^{p^{m}}\right) \geq p^{k} q .
$$

Hence $v_{\bar{z}, \mathcal{O}}\left(s^{p^{m}}\right) \geq v_{\bar{z}, \mathcal{O}}\left(r^{p^{m}}\right)+p^{k} \geq p^{k} q+p^{k}=p^{k}(q+1)$, so $s^{p^{m}} \in z^{p^{k}(q+1)} \mathcal{O}=x^{q+1} \mathcal{O}=J(\mathcal{O})^{a(q+1)} \subseteq$ $J(\mathcal{O})^{n+1}$.

Therefore $v_{\mathfrak{q}}\left(s^{p^{m}}\right) \geq n+1>n=v_{\mathfrak{q}}\left(r^{p^{m}}\right)$.

Furthermore, for all $l \in \mathbb{N}, v_{\mathfrak{q}}\left(s^{p^{m+l}}\right) \geq p^{l} v_{\mathfrak{q}}\left(s^{p^{m}}\right)>p^{l} v_{\mathfrak{q}}\left(r^{p^{m}}\right)=v_{\mathfrak{q}}\left(r^{p^{m+l}}\right)$ - the last equality holds since $r^{p^{m}}$ is $v_{\mathfrak{q}}$-regular.

Hence $v_{\mathfrak{q}}\left(s^{p^{n}}\right)>v_{\mathfrak{q}}\left(r^{p^{n}}\right)$ for all sufficiently high $n$ as required.

\subsection{Growth Rates}

Since we are usually only interested in convergence, it makes sense to consider the growth of elements values as they are raised to high powers.

Definition 3.2. Let $Q$ be a ring with a filtration $v: Q \rightarrow \mathbb{Z} \cup\{\infty\}$. Define $\rho: Q \rightarrow \mathbb{R} \cup\{\infty\}, x \rightarrow \lim _{n \rightarrow \infty} \frac{v\left(x^{n}\right)}{n}$. This is the growth rate function of $Q$ with respect to $v$, the proof of [5, Lemma 1] shows that this is well defined.

Lemma 3.7. Let $Q$ be a ring with a filtration $v: Q \rightarrow \mathbb{Z} \cup\{\infty\}$, and let $\rho$ be the corresponding growth rate function. Then for all $x, y \in Q$ :

i. $\rho\left(x^{n}\right)=n \rho(x)$ for all $n \in \mathbb{N}$.

ii. If $x$ and $y$ commute then $\rho(x+y) \geq \min \{\rho(x), \rho(y)\}$ and $\rho(x y) \geq \rho(x)+\rho(y)$.

iii. $\rho(x) \geq v(x)$ and $\rho$ is invariant under conjugation.

$i v$. If $Q$ is simple and artinian and $v$ is separated, then $\rho(x)=\infty$ if and only if $x$ is nilpotent.

$v$. If $x$ is $v$-regular and commutes with $y$, then $\rho(x)=v(x)$ and $\rho(x y)=v(x)+\rho(y)$.

Proof. $i$ and $i$ are given by the proof of [5, Lemma 1].

iii. For each $n \in \mathbb{N}, \frac{v\left(x^{n}\right)}{n} \geq \frac{n v(x)}{n}=v(x)$, and so $\rho(x) \geq v(x)$.

Given $u \in Q^{\times}, \rho\left(u x u^{-1}\right)=\lim _{n \rightarrow \infty} \frac{v\left(\left(u x u^{-1}\right)^{n}\right)}{n}=\lim _{n \rightarrow \infty} \frac{v\left(u\left(x^{n}\right) u^{-1}\right)}{n} \geq \lim _{n \rightarrow \infty} \frac{v(u)+v\left(x^{n}\right)+v\left(u^{-1}\right)}{n}=\lim _{n \rightarrow \infty} \frac{v\left(x^{n}\right)}{n}+$ $\lim _{n \rightarrow \infty} \frac{v(u)}{n}+\lim _{n \rightarrow \infty} \frac{v\left(u^{-1}\right)}{n}=\rho(x)$.

Hence $\rho(x)=\rho\left(u^{-1} u x u^{-1} u\right) \geq \rho\left(u x u^{-1}\right) \geq \rho(x)$ - forcing equality. Therefore $\rho$ is invariant under conjugation.

$i v$. Clearly if $x$ is nilpotent then $\rho(x)=\infty$.

First suppose that $x$ is a unit, then for any $y \in Q, v(y)=v\left(x^{-1} x y\right) \geq v\left(x^{-1}\right)+v(x y)$, and so $v(x y) \leq v(y)-$ $v\left(x^{-1}\right)$. It follows using induction that for all $n \in \mathbb{N}, v\left(x^{n} y\right) \leq v(y)-n v\left(x^{-1}\right)$, and hence $\frac{v\left(x^{n} y\right)}{n} \leq \frac{v(y)}{n}-v\left(x^{-1}\right)$.

Taking $y=1$, it follows easily that $\rho(x) \leq-v\left(x^{-1}\right)$, and since $v$ is separated, this is less than $\infty$.

Now, since $Q$ is simple and artinian, we have that $Q \cong M_{l}(K)$ for some division $\operatorname{ring} K, l \in \mathbb{N}$. So applying Fitting's Lemma [9, section 3.4], we can find a unit $u \in Q^{\times}$such that $u x u^{-1}$ has standard Fitting block form $\left(\begin{array}{cc}A & 0 \\ 0 & B\end{array}\right)$, where $A$ and $B$ are square matrices over $K$, possibly empty, $A$ is invertible and $B$ is nilpotent.

If $x$ is not nilpotent then $u x u^{-1}$ is not nilpotent, and hence $A$ is non-empty. Therefore, $\rho\left(u x u^{-1}\right)=\rho(A)$, and since $A$ is invertible, $\rho(A)<\infty$. So by part iii, $\rho(x)=\rho\left(u x u^{-1}\right)<\infty$.

$v$. Since $x$ is $v$-regular, $v\left(x^{n}\right)=n v(x)$ for all $n$, so clearly $\rho(x)=v(x)$. Also, $\rho(x y)=\lim _{n \rightarrow \infty} \frac{v\left((x y)^{n}\right)}{n}=$ $\lim _{n \rightarrow \infty} \frac{v\left(x^{n} y^{n}\right)}{n}=\lim _{n \rightarrow \infty} \frac{v\left(x^{n}\right)+v\left(y^{n}\right)}{n}=\lim _{n \rightarrow \infty} \frac{v\left(\left(x^{n}\right)\right.}{n}+\lim _{n \rightarrow \infty} \frac{v\left(y^{n}\right)}{n}=\rho(x)+\rho(y)=v(x)+v(y)$. 
So let $G$ be a non-abelian $p$-valuable, abelian-by-procyclic group with principal subgroup $H$, procyclic element $X$, and let $P$ be a faithful prime ideal of $k G$. Fix $v$ a non-commutative valuation on $Q\left(\frac{k G}{P}\right)$ such that the natural map $\tau: k G \rightarrow Q\left(\frac{k G}{P}\right)$ is continuous, and let $\rho$ be the growth rate function of $v$.

Recall the function $u=z\left(\varphi^{p^{m_{1}}}\right): H \rightarrow H$ defined in section 2.1, where $\varphi$ is conjugation by $X$. Define $\lambda:=\inf \{\rho(\tau(u(h)-1)): h \in G\}$. Since we know that for all $h \in H, u(h)=u_{0}(h)^{p^{m_{1}}}$ and $\left.v\left(\tau(h-1)^{p^{m}}\right)\right) \geq 1$ for sufficiently high $m$, we may choose $m_{1}$ such that $v(\tau(u(h)-1)) \geq 1$, and hence $\lambda \geq 1$.

Lemma 3.8. $\lambda<\infty$ and $\lambda$ is attained as the growth rate of $\tau(u(h)-1)$ for some $h \in H$.

Proof. Suppose that $\lambda=\infty$, i.e. $\rho(\tau(u(h)-1))=\infty$ for all $h \in G$.

By Lemma 3.7 (iv), it follows that $\tau(u(h)-1)$ is nilpotent for all $h \in H$, and hence $u(h)^{p^{m}}-1 \in P$ for sufficiently high $m$.

So since $P$ is faithful, it follows that $u(h)^{p^{m}}=u_{0}(h)^{p^{m_{1}+m}}=1$, and hence $u_{0}(h)=1$ by the torsionfree property, and this holds for all $h \in H$.

But in this case, $1=u_{0}(h)=\exp ([\log (X), \log (h)])$, so $[\log (X), \log (h)]=0$ in $\log (\operatorname{Sat}(G))$, and it follows that $\log (\operatorname{Sat}(G))$ is abelian, and hence $G$ is abelian - contradiction.

Therefore $\lambda$ is finite, and it is clear that for any basis $\left\{h_{1}, \cdots, h_{d}\right\}$ for $H, \lambda=\min \left\{\rho\left(\tau\left(u\left(h_{i}\right)-1\right)\right): i=\right.$ $1, \cdots, d\}$, hence it is attained.

\subsection{Growth Preserving Polynomials}

We will now see the first example of proving a control theorem using our Mahler expansions.

Consider a polynomial of the form $f(t)=a_{0} t+a_{1} t^{p}+\cdots+a_{r} t^{p^{r}}$, where $a_{i} \in \tau(k H)$ and $r \geq 0$, we call $r$ the $p$-degree of $f$.

Then $f: \tau(k H) \rightarrow \tau(k H)$ is an $\mathbb{F}_{p}$-linear map.

Recall the definition of $\lambda \geq 1$ from section 3.3, which is implicit in the following definition.

Definition 3.3. We say that $f(t)=a_{0} t+a_{1} t^{p}+\cdots+a_{r} t^{p^{r}}$ is a growth preserving polynomial, or GPP, if:

i. $\rho(f(q)) \geq p^{r} \lambda$ for all $q \in \tau(k H)$ with $\rho(q) \geq \lambda$.

ii. $\rho(f(q))>p^{r} \lambda$ for all $q \in \tau(k H)$ with $\rho(q)>\lambda$.

We say that a GPP $f$ is trivial if for all $q=\tau(u(h)-1), \rho(f(q))>p^{r} \lambda$.

Furthermore, $f$ is a special GPP if $f$ is not trivial, and for any $q=\tau(u(h)-1)$ with $\rho(f(q))=p^{r} \lambda$, we have that $f(q)^{p^{k}}$ is v-regular for sufficiently high $k$.

Example: $f(t)=t$ is clearly a GPP. In general it need not be special, and it is not trivial, because if $\rho(q)=\lambda$ then $\rho(f(q))=\rho(q)=\lambda$.

For any growth preserving polynomial $f(t)$, define $K_{f}:=\left\{h \in H: \rho(f(\tau(u(h)-1)))>p^{r} \lambda\right\}$.

Lemma 3.9. If $f(t)$ is a GPP, then $K_{f}$ is an open subgroup of $H$ containing $H^{p}$. Moreover, $K_{f}=H$ if and only if $f$ is trivial.

Proof. Firstly, it is clear from the definition that $K_{f}=H$ if and only if $\rho(f(\tau(u(h)-1)))>p^{r} \lambda$ for all $h \in H$, i.e. if and only if $f$ is trivial.

Given $h, h^{\prime} \in H$, let $q=\tau(u(h)-1), q^{\prime}=\tau\left(u\left(h^{\prime}\right)-1\right)$. Then

$$
\left.\tau\left(u\left(h h^{\prime}\right)-1\right)=\tau\left((u(h)-1)\left(u\left(h^{\prime}\right)-1\right)+(u(h)-1)+\left(u\left(h^{\prime}\right)-1\right)\right)\right)=q q^{\prime}+q+q^{\prime}
$$

Therefore $f\left(\tau\left(u\left(h h^{\prime}\right)-1\right)\right)=f\left(q q^{\prime}\right)+f(q)+f(q)$ using $\mathbb{F}_{p^{-}}$-linearity of $f$. But $\rho(q), \rho\left(q^{\prime}\right) \geq \lambda$ by the definition of $\lambda$, so $\rho\left(q q^{\prime}\right) \geq 2 \lambda>\lambda$, so by the definition of a GPP, $\rho\left(f\left(q q^{\prime}\right)\right)>p^{r} \lambda$. 
We know that $\rho(f(q)), \rho\left(f\left(q^{\prime}\right)\right)>p^{r} \lambda$, thus $\rho\left(f\left(q_{1} q_{2}\right)+f\left(q_{1}\right)+f\left(q_{2}\right)\right)>p^{r} \lambda$. Therefore, $\rho\left(f\left(\tau\left(u\left(h h^{\prime}\right)-1\right)\right)\right)>$ $p^{r} \lambda$ and $h h^{\prime} \in K_{f}$ as required.

Also, $\tau\left(u\left(h^{-1}\right)-1\right)=-\tau\left(u\left(h^{-1}\right)\right) \tau(u(h)-1)=-\tau\left(u\left(h^{-1}\right)-1\right) \tau(u(h)-1)-\tau(u(h)-1)$, so by the same argument it follows that $h^{-1}$ in $K_{f}$, and $K_{f}$ is a subgroup of $H$.

Finally, for any $h \in H \rho\left(\tau\left(u\left(h^{p}\right)-1\right)\right)=\rho\left(\tau(u(h)-1)^{p}\right) \geq p \lambda>\lambda$, hence $\rho\left(f\left(\tau\left(u\left(h^{p}\right)-1\right)\right)\right)>p^{r} \lambda$ and $h^{p} \in K_{f}$. Therefore $K_{f}$ contains $H^{p}$ and $K_{f}$ is an open subgroup of $H$.

In particular, for $f(t)=t$, let $K:=K_{f}=\{h \in H: \rho(\tau(u(h)-1))>\lambda\}$. Then $K$ is a proper open subgroup of $H$ containing $H^{p}$.

Given a polynomial $f(t)=a_{0} t+a_{1} t^{p}+\cdots+a_{r} t^{p^{r}}$, and a basis $\left\{h_{1}, \cdots, h_{d}\right\}$ for $H$, let $q_{i}=\tau\left(u\left(h_{i}\right)-1\right)$, and recall our expression (6) from section 2 :

$$
0=f\left(q_{1}\right)^{p^{m}} \tau \partial_{1}(y)+\ldots .+f\left(q_{d}\right)^{p^{m}} \tau \partial_{d}(y)+O\left(f(q)^{p^{m}}\right)
$$

Where $v\left(q^{p^{m}}\right) \geq 2 v\left(q_{i}^{m}\right)$ for all $m$, and hence $\rho(q)>\rho\left(q_{i}\right) \geq \lambda$. Then if $f$ is a GPP, it follows that $\rho(f(q))>p^{r} \lambda$, and hence $v\left(f(q)^{p^{m}}\right)>p^{m+r} \lambda$ for $m>>0$.

For the rest of this section, fix a non-trivial GPP $f$ of $p$ degree $r$. Then $K_{f}$ is a proper open subgroup of $H$ containing $H^{p}$ by Lemma 3.9, so fix a basis $\left\{h_{1}, \cdots, h_{d}\right\}$ for $H$ such that $\left\{h_{1}^{p}, \cdots, h_{t}^{p}, h_{t+1}, \cdots, h_{d}\right\}$ is an ordered basis for $K_{f}$.

Set $q_{i}:=\tau\left(u\left(h_{i}\right)-1\right)$ for each $i$, so that $\rho\left(f\left(q_{i}\right)\right)=p^{r} \lambda$ for $i \leq t$ and $\rho\left(f\left(q_{i}\right)\right)>p^{r} \lambda$ for $i>t$, and define:

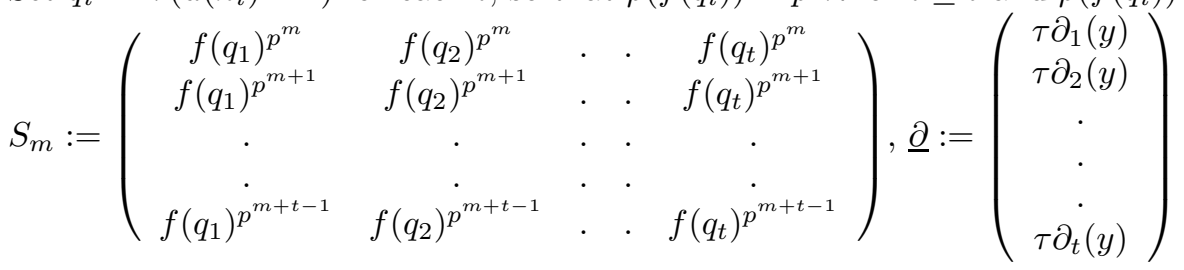

Then we can rewrite our expression as:

$$
0=S_{m} \underline{\partial}+\left(\begin{array}{c}
O\left(f(q)^{p^{m}}\right) \\
O\left(f(q)^{p^{m+1}}\right) \\
\cdot \\
\cdot \\
\cdot \cdot \\
O\left(f(q)^{p^{m+t-1}}\right)
\end{array}\right)
$$

And multiplying by $\operatorname{adj}\left(S_{m}\right)$ gives:

$$
0=\operatorname{det}\left(S_{m}\right) \underline{\partial}+\operatorname{adj}\left(S_{m}\right)\left(\begin{array}{c}
O\left(f(q)^{p^{m}}\right) \\
O\left(f(q)^{p^{m+1}}\right) \\
\cdot \\
\cdot \\
\cdot \\
O\left(f(q)^{p^{m+t-1}}\right)
\end{array}\right)
$$

Lemma 3.10. Suppose that $f$ is special. Then for each $i, j \leq t$, the $(i, j)$-entry of adj $\left(S_{m}\right)$ has value at least $\frac{p^{t}-1}{p-1} p^{m+r} \lambda-p^{m+r+j-1} \lambda$ for sufficiently high $m$.

Proof. By definition, the $(i, j)$-entry of $\operatorname{adj}\left(S_{m}\right)$ is (up to sign) the determinant of the matrix $\left(S_{m}\right)_{i, j}$ obtained by removing the $j^{\prime}$ th row and $i$ th column of $S_{m}$. This determinant is a sum of elements of the form:

$$
f\left(q_{k_{1}}\right)^{p^{m}} f\left(q_{k_{2}}\right)^{p^{m+1}} \cdots f\left(\widehat{\left.q_{k_{j}}\right)^{p^{m+j-1}}} \cdots f\left(q_{k_{t}}\right)^{p^{m+t-1}}\right.
$$

For $k_{i} \leq t$, where the hat indicates that the $j$ 'th term in this product is omitted. 
Since $f$ is special, $f\left(q_{k_{s}}\right)^{p^{m}}$ is $v$-regular for $m>>0$. So since $\rho\left(f\left(q_{k_{s}}\right)\right)=p^{r} \lambda$, it follows that $f\left(q_{k_{s}}\right)$ is $v$-regular of value $p^{m+r} \lambda$.

Therefore, this $(i, j)$-entry has value at least $\left(1+p+\cdots+\widehat{p^{j-1}}+\cdots+p^{t-1}\right) p^{m+r} \lambda$

$=\frac{p^{t}-1}{p-1} p^{m+r} \lambda-p^{m+r+j-1} \lambda$.

Lemma 3.11. Let $\Delta:=\prod_{\alpha \in \mathbb{P}^{t-1} \mathbb{F}_{p}}\left(\alpha_{1} f\left(q_{1}\right)+\cdots+\alpha_{t} f\left(q_{t}\right)\right)$.

Then there exists $\delta \in \tau(k H)$, which is a product of length $\frac{p^{t}-1}{p-1}$ in elements of the form $f(\tau(u(h)-1))$, $h \in H \backslash K_{f}$, such that $\rho(\Delta-\delta)>\frac{p^{t}-1}{p-1} p^{r} \lambda$.

Proof. For each $\alpha \in \mathbb{F}_{p}^{t} \backslash\{0\}$, we have that $\alpha_{1} f\left(q_{1}\right)+\cdots+\alpha_{t} f\left(q_{t}\right)=f\left(\alpha_{1} q_{1}+\cdots+\alpha_{t} q_{t}\right)$ using linearity of $f$.

Using expansions inside $k H$, we see that $\alpha_{1} q_{1}+\cdots+\alpha_{t} q_{t}=\tau\left(u\left(h_{1}^{\alpha_{1}} \cdots h_{t}^{\alpha_{t}}\right)-1\right)+O\left(q_{j} q_{k}\right)$, and hence:

$$
f\left(\alpha_{1} q_{1}+\cdots+\alpha_{t} q_{t}\right)=f\left(\tau\left(u\left(h_{1}^{\alpha_{1}} \cdots h_{t}^{\alpha_{t}}\right)-1\right)\right)+O\left(f\left(q_{j} q_{k}\right)\right)
$$

So setting $h_{i, \alpha}:=u\left(h_{1}^{\alpha_{1}} \cdots h_{t}^{\alpha_{t}}\right)$. So since $\alpha_{i} \neq 0$ for some $i$, it follows from $\mathbb{F}_{p}$-linear independence of $h_{1}, \cdots, h_{t}$ modulo $K_{f}$, that $\rho\left(f\left(\tau\left(u\left(h_{i, \alpha}\right)-1\right)\right)\right)=p^{r} \lambda$, and hence $h_{i, \alpha} \in H \backslash K_{f}$.

Set $\delta:=\prod_{\alpha \in \mathbb{P}^{t}-1 \mathbb{F}_{p}} f\left(\tau\left(u\left(h_{i, \alpha}\right)-1\right)\right)$. Then $\Delta=\prod_{\alpha \in \mathbb{P}^{t-1} \mathbb{F}_{p}}\left(\alpha_{1} f\left(q_{1}\right)+\cdots+\alpha_{t} f\left(q_{t}\right)\right)$

$=\prod_{\alpha \in \mathbb{P}^{t-1} \mathbb{F}_{p}} f\left(\tau\left(u\left(h_{i, \alpha}\right)-1\right)\right)+O\left(f\left(q_{j} q_{k}\right)\right)=\delta+\epsilon$

Where $\epsilon$ is a sum of products over all $i, \alpha$ in $f\left(\tau\left(u\left(h_{i, \alpha}\right)-1\right)\right)$ and $O\left(f\left(q_{j} q_{k}\right)\right)$, with each product containing at least one $O\left(f\left(q_{j} q_{k}\right)\right)$.

Since the length of each of these products is $\frac{p^{t}-1}{p-1}$, and each term has growth rate at least $p^{r} \lambda$, with one or more having growth rate strictly greater than $p^{r} \lambda$, it follows that $\rho(\epsilon)>\frac{p^{t}-1}{p-1} p^{r} \lambda$ as required.

Theorem 3.12. Suppose that there exists a special growth preserving polynomial $f$. Then $P$ is controlled by a proper open subgroup of $G$.

Proof. Since $f$ is special, we have that for some $k>0 f\left(q_{i}\right)^{p^{k}}$ is $v$-regular for each $i \leq t$, and thus $v\left(f\left(q_{i}\right)^{p^{k}}\right)=\rho\left(f\left(q_{i}\right)^{p^{k}}\right)=p^{r+k} \lambda$ and for all $m \geq k, v\left(f\left(q_{i}\right)^{p^{m}}\right)=p^{r+m} \lambda$.

Also, since $\rho(f(q))>p^{r} \lambda$, we can choose $c>0$ such that $\rho(f(q))>p^{r} \lambda+c$, and hence $v\left(f(q)^{p^{m}}\right)>p^{m+r} \lambda+p^{m} c$ for sufficiently high $m$.

Consider our Mahler expansion (8):

$$
0=\operatorname{det}\left(S_{m}\right) \underline{\partial}+\operatorname{adj}\left(S_{m}\right)\left(\begin{array}{c}
O\left(f(q)^{p^{m}}\right) \\
O\left(f(q)^{p^{m+1}}\right) \\
\cdot \\
\cdot \\
\cdot \\
O\left(f(q)^{p^{m+t-1}}\right)
\end{array}\right)
$$

We will analyse this expression to prove that $\tau \partial_{i}(P)=0$ for all $i \leq t$, and it will follow from Proposition 2.4 that $P$ is controlled by a proper open subgroup of $G$ as required.

Consider the $i$ 'th entry of the vector

$$
\operatorname{adj}\left(S_{m}\right)\left(\begin{array}{c}
O\left(f(q)^{p^{m}}\right) \\
O\left(f(q)^{p^{m+1}}\right) \\
\cdot \\
\cdot \\
\cdot \\
O\left(f(q)^{p^{m+t-1}}\right)
\end{array}\right)
$$


This has the form $\operatorname{adj}\left(S_{m}\right)_{i, 1} O\left(f(q)^{p^{m}}\right)+\operatorname{adj}\left(S_{m}\right)_{i, 2} O\left(f(q)^{p^{m+1}}\right)+\cdots+\operatorname{adj}\left(S_{m}\right)_{i, t} O\left(f(q)^{p^{m+t-1}}\right)$.

By Lemma 3.10, we know that $v\left(\operatorname{adj}\left(S_{m}\right)_{i, j}\right) \geq \frac{p^{t}-1}{p-1} p^{m+r} \lambda-p^{m+r+j-1} \lambda$ for $m>>0$, and hence:

$v\left(\operatorname{adj}\left(S_{m}\right)_{i, j} O\left(f(q)^{p^{m+j-1}}\right)\right) \geq v\left(\operatorname{adj}\left(S_{m}\right)_{i, j}\right)+v\left(O\left(f(q)^{p^{m+j-1}}\right)\right)$

$\geq \frac{p^{t}-1}{p-1} p^{m+r} \lambda-p^{m+r+j-1} \lambda+p^{m+r+j-1} \lambda+p^{m+j-1} c=\frac{p^{t}-1}{p-1} p^{m+r} \lambda+p^{m+j-1} c$ for each $j$.

Hence this $i$ 'th entry has value at least $\frac{p^{t}-1}{p-1} p^{m+r} \lambda+p^{m} c$.

Therefore, the $i$ 'th entry of our expression (8) has the form $0=\operatorname{det}\left(S_{m}\right) \tau \partial_{i}(y)+\epsilon_{i, m}$, where $v\left(\epsilon_{i, m}\right) \geq$ $\frac{p^{t}-1}{p-1} p^{m+r} \lambda+p^{m} c$.

Now, take $\Delta:=\operatorname{det}\left(S_{0}\right)$, and it follows that $\operatorname{det}\left(S_{m}\right)=\Delta^{p^{m}}$ for each $m$. Also, using [4, Lemma 1.1(ii)] we see that:

$$
\Delta=\beta \cdot \prod_{\alpha \in \mathbb{P}^{t-1} \mathbb{F}_{p}}\left(\alpha_{1} f\left(q_{1}\right)+\cdots+\alpha_{t} f\left(q_{t}\right)\right) \text { for some } \beta \in \mathbb{F}_{p} .
$$

Therefore, by Lemma 3.11 we can find an element $\delta \in \tau(k H)$, which up to scalar multiple is a product of length $\frac{p^{t}-1}{p-1}$ in elements of the form $f(\tau(u(h)-1))$, with $\rho(f(\tau(u(h)-1)))=p^{r} \lambda$ for each $h$, such that $\rho(\Delta-\delta)>\frac{p^{t}-1}{p-1} p^{r} \lambda$.

Hence we can find $c^{\prime}>0$ such that for all $m>>0, v\left((\Delta-\delta)^{p^{m}}\right) \geq \frac{p^{t}-1}{p-1} p^{m+r} \lambda+p^{m} c^{\prime}$.

Therefore, $0=\operatorname{det}\left(S_{m}\right) \tau \partial_{i}(y)+\epsilon_{i, m}=\Delta^{p^{m}} \tau \partial_{i}(y)+\epsilon_{i, m}=\delta^{p^{m}} \tau \partial_{i}(y)+(\Delta-\delta)^{p^{m}} \tau \partial_{i}(y)+\epsilon_{i, m}$.

Again, since $f$ is a special GPP, if $h \in H$ and $\rho(f(\tau(u(h)-1)))=p^{r} \lambda$, it follows that $f(\tau(u(h)-1))^{p^{k}}$ is $v$-regular for $k>>0$.

So since $\delta$ is a product of $\frac{p^{t}-1}{p-1}$ elements of the form $f(\tau(u(h)-1))$ of growth rate $p^{r} \lambda$, it follows that for some $k \in \mathbb{N}, \delta^{p^{k}}$ is $v$-regular of value $\frac{p^{t}-1}{p-1} p^{r+k} \lambda$.

Therefore for all $m \geq k, \delta^{p^{m}}$ is $v$-regular of value $\frac{p^{t}-1}{p-1} p^{r+m} \lambda$, and dividing out by $\delta^{p^{m}}$ gives that for each $i=1, \cdots, t$ :

$$
0=\tau \partial_{i}(y)+\delta^{-p^{m}}(\Delta-\delta)^{p^{m}} \tau \partial_{i}(y)+\delta^{-p^{m}} \epsilon_{i, m}
$$

But for $m>>, v\left(\delta^{-p^{m}}(\Delta-\delta)^{p^{m}}\right) \geq \frac{p^{t}-1}{p-1} p^{m+r} \lambda+p^{m} c^{\prime}-\frac{p^{t}-1}{p-1} p^{m+r} \lambda=p^{m} c^{\prime}$, and $v\left(\delta^{-p^{m}} \epsilon_{i, m}\right) \geq$ $\frac{p^{t}-1}{p-1} p^{m+r} \lambda+p^{m} c-\frac{p^{t}-1}{p-1} p^{m+r} \lambda=p^{m} c$, hence the right hand side of this expression converges to $\tau \partial_{i}(y)$.

Therefore, $\tau \partial_{i}(y)=0$, and since this holds for all $y \in P$, we have that $\tau \partial_{i}(P)=0$ for each $i=1, \cdots, t$.

So to prove Theorem 1.5, it remains only to prove the existence of a special growth preserving polynomial. We will construct such a polynomial in section 6 .

\section{Central Simple algebras}

Suppose that $G$ is an abelian-by-procyclic group, and $P$ is a faithful prime ideal of $k G$. Since $Q:=Q\left(\frac{k G}{P}\right)$ is simple, its centre is a field. Recall that the proof of Theorem 1.5 will split into two parts, when $Q$ is finite dimensional over its centre, and when it is not.

In this section, we deal with the former case. So we will assume throughout that $Q$ is finitely generated over its centre, i.e. is a central simple algebra.

\subsection{Isometric embedding}

Fix a non-commutative valuation $v$ on $Q$. Then by definition, the completion $\widehat{Q}$ of $Q$ with respect to $v$ is isomorphic to $M_{n}(Q(D))$ for some complete, non-commutative DVR $D$. 
But since $Q$ is finite dimensional over its centre, the same property holds for $\widehat{Q}$, and hence $Q(D)$ is finite dimensional over its centre.

Let $F:=Z(Q(D)), s:=\operatorname{dim}_{F}(Q(D)), R:=F \cap D$. Then $F$ is a field, $R$ is a commutative DVR, and $Q(D) \cong F^{s}$. Let $\pi \in R$ be a uniformiser, and suppose that $v(\pi)=t>0$.

Lemma 4.1. Let $\left\{y_{1}, \cdots, y_{s}\right\}$ be an $F$-basis for $Q(D)$ with $0 \leq v\left(y_{i}\right) \leq t$ for all $i$. Then there exists $l \in \mathbb{N}$ such that if $v\left(r_{1} y_{1}+\cdots+r_{s} y_{s}\right) \geq l$ then $v\left(r_{i}\right)>0$ for some $i$ with $r_{i} \neq 0$.

Proof. First, note that the field $F$ is complete with respect to the non-archimedian valuation $v$, and $Q(D) \cong F^{s}$ carries two filtrations as a $F$-vector space, which both restrict to $v$ on $F$. One is the natural valuation $v$ on $Q(D)$, the other is given by $v_{0}\left(r_{1} y_{1}+\ldots .+r_{s} y_{s}\right)=\min \left\{v\left(r_{i}\right): i=1, \ldots, s\right\}$.

But it follows from [6. Proposition 2.27] that any two norms on $F^{s}$ are topologically equivalent. Hence $v$ and $v_{0}$ induce the same topology on $Q(D)$.

It is easy to see that any subspace of $F^{s}$ is closed with respect to $v_{0}$, and hence also with respect to $v$.

So, suppose for contradiction that for each $m \in \mathbb{N}$, there exist $r_{i, m} \in K$, not all zero, with $v\left(r_{i, m}\right) \leq 0$ if $r_{i, m} \neq 0$, and $v\left(r_{1, m} y_{1}+\ldots .+r_{s, m} y_{s}\right) \geq m$.

Then there exists $i$ such that $r_{i, m} \neq 0$ for infinitely many $m$, and we can assume without loss of generality that $i=1$. So from now on, we assume that $r_{1, m} \neq 0$ for all $m$.

Dividing out by $r_{1, m}$ gives that $v\left(y_{1}+t_{2, m} y_{2}+\ldots .+t_{s, m} y_{s}\right) \geq m-v\left(r_{1, m}\right) \geq m$, where $t_{i, m}=r_{1, m}^{-1} r_{i, m} \in K$.

Hence $\lim _{m \rightarrow \infty}\left(y_{1}+t_{2, m} y_{2}+\ldots .+t_{s, m} y_{s}\right)=0$, and thus $\lim _{n \rightarrow \infty}\left(t_{2, m} y_{2}+\ldots .+t_{s, m} y_{s}\right)$ exists and equals $-y_{1}$. But since $\operatorname{Span}_{F}\left\{y_{2}, \ldots, y_{s}\right\}$ is closed in $F^{n}$, this means that $y_{1} \in \operatorname{Span}_{F}\left\{y_{2}, \ldots ., y_{s}\right\}$ - contradiction.

Proposition 4.2. There exists a basis $\left\{x_{1}, \ldots, x_{s}\right\} \subseteq D$ for $Q(D)$ over $F$ such that $D=R x_{1} \oplus \ldots \oplus R x_{s}$.

Proof. It is clear that we can find an $F$-basis $\left\{y_{1}, \ldots, y_{s}\right\} \subseteq D$ for $Q(D)$ such that $v\left(y_{i}\right)<t$ for all $i$, just by rescaling elements of some arbitrary basis.

Therefore, by Lemma 4.1, there exists $l \in \mathbb{N}$ such that if $v\left(r_{1} y_{1}+\cdots+r_{s} t_{s}\right) \geq l$ then $v\left(r_{i}\right)>0$ for some $i$ with $r_{i} \neq 0$.

Choose $m \in \mathbb{N}$ such that $t m>l$. Then given $x \in D \backslash\{0\}, v(x) \geq 0$ so $v\left(\pi^{m} x\right) \geq t m>l$. So if $\pi^{m} x=r_{1} y_{1}+\ldots .+r_{s} y_{s}$ then $v\left(r_{i}\right) \geq 0$ for some $r_{i} \neq 0$.

It follows from an easy inductive argument that $\pi^{m s} x \in R y_{1} \oplus \ldots . \oplus R y_{s}$, and hence $\pi^{m s} D \subseteq R y_{1} \oplus \ldots . \oplus R y_{s}$.

But $R y_{1} \oplus \ldots \oplus \oplus R y_{s}$ is a free $R$-module, and $R$ is a commutative PID, hence any $R$-submodule is also free. Hence $\pi^{m s} D=R\left(\pi^{m s} x_{1}\right) \oplus \ldots \oplus R\left(\pi^{m s} x_{e}\right)$ for some $x_{i} \in D$.

It follows easily that $e=s,\left\{x_{1}, \ldots, x_{s}\right\}$ is an $F$-basis for $Q(D)$, and $D=R x_{1} \oplus \ldots \oplus R x_{s}$.

Now, we restrict our non-commutative valuation $v$ on $\widehat{Q} \cong M_{n}(Q(D))$ to $Q(D)$, and by definition this is the natural $J(D)$-adic valuation.

Using Proposition 4.2, we fix a basis $\left\{x_{1}, \cdots, x_{s}\right\} \subseteq D$ for $Q(D)$ over $F$, with $D=R x_{1} \oplus R x_{2} \oplus \cdots \oplus R x_{s}$.

Proposition 4.3. Let $F^{\prime}$ be any finite extension of $F$; then $v$ extends to $F^{\prime}$. Let $v^{\prime}$ be the standard matrix filtration of $M_{s}\left(F^{\prime}\right)$ with respect to $v$.

Then there is a continuous embedding of F-algebras $\phi: Q(D) \hookrightarrow M_{s}\left(F^{\prime}\right)$ such that

$$
v^{\prime}(\phi(x)) \leq v(x)<v^{\prime}(\phi(x))+2 t \text { for all } x \in Q(D) .
$$

Hence applying the functor $M_{n}$ to $\phi$ gives us a continuous embedding $M_{n}(\phi): \widehat{Q} \hookrightarrow M_{n s}\left(F^{\prime}\right)$ such that $v^{\prime}\left(M_{n}(\phi)(x)\right) \leq v(x)<v^{\prime}\left(M_{n}(\phi)(x)\right)+2 t$ for all $x \in \widehat{Q}$.

Proof. It is clear that the embedding $F \rightarrow F^{\prime}$ is an isometry, so it suffices to prove the result for $F^{\prime}=F$.

Again, define $v_{0}: Q(D) \rightarrow \mathbb{Z} \cup\{\infty\}, \sum_{i=1}^{s} r_{i} x_{i} \mapsto \min \left\{v\left(r_{i}\right): i=1, \ldots, s\right\}$, it is readily checked that this is a separated filtration of $F$-vector spaces, and clearly $v(x) \geq v_{0}(x)$ for all $x \in Q(D)$. 
Then if $x=r_{1} x_{1}+\ldots .+r_{s} x_{s} \in Q(D)$ with $0 \leq v(x)<t$, then $v\left(r_{i}\right) \geq 0$ for all $i$ because $D$ is an $R$-lattice by Lemma 4.2. Since $v\left(x_{i}\right) \geq 0$ for all $i, v\left(r_{j}\right)<t$ for some $j$, so since $r_{j} \in R$, this means that $v\left(r_{j}\right)=0$, and hence $v_{0}(x)=0$.

So if $x \in Q(D)$ with $v(x)=l$, then $a t \leq l<(a+1) t$ where $a=\left\lfloor\frac{l}{t}\right\rfloor$, and hence $0 \leq v\left(\pi^{-a} x\right)<l$, so $v_{0}\left(\pi^{-a} x\right)=0$.

Thus $\pi^{-a} x=r_{1} x_{1}+\ldots+r_{s} x_{s}$ with $v\left(r_{i}\right) \geq 0$ for all $i, v\left(r_{j}\right)=0$ for some $i$, and hence $v_{0}(x)=t a$, so $v(x)<v_{0}(x)+t$.

So it follows that $v_{0}(x) \leq v(x)<v_{0}(x)+t$ for all $x \in Q(D)$, in particular $0 \leq v\left(x_{i}\right)<t$ for all $i$. Hence the identity map $(Q(D), v) \rightarrow\left(Q(D), v_{0}\right)$ is bounded.

Now, consider the map $\phi: Q(D) \rightarrow \operatorname{End}_{F}(Q(D)), x \mapsto(Q(D) \rightarrow Q(D), d \mapsto x \cdot d)$, this is an injective $F$ algebra homomorphism.

Also, $\operatorname{End}_{F}(Q(D))$ carries a natural filtration of $F$-algebras given by

$$
v^{\prime}(\psi):=\min \left\{v_{0}\left(\psi\left(x_{i}\right)\right): i=1, \ldots, s\right\} \text { for each } \psi \in \operatorname{End}_{F}(Q(D)) .
$$

Using the isomorphism $\operatorname{End}_{F}(Q(D)) \cong M_{s}(F)$, this is just the standard matrix filtration, and it is readily seen that

$$
v^{\prime}(\psi)=\inf \left\{v_{0}(\psi(d)): d \in Q(D), 0 \leq v^{\prime}(d)<t\right\} \text { for all } \psi \in \operatorname{End}_{F}(Q(D)) .
$$

So if $v_{0}(x)=r$ then since $v_{0}(1)=0$ and $v_{0}(\phi(x)(1))=v_{0}(x \cdot 1)=r$, it follows that $v^{\prime}(\phi(x)) \leq r$. But for all $i=1, \ldots, s$ :

$$
v_{0}\left(x \cdot x_{i}\right)>v\left(x \cdot x_{i}\right)-t \geq r+v\left(x_{i}\right)-t \geq r-t, \text { hence } v^{\prime}(\phi(x)) \geq r-t .
$$

Therefore $v^{\prime}(\phi(x)) \leq v_{0}(x) \leq v^{\prime}(\phi(x))+t$ for all $x$, so $\phi$ is bounded, and hence continuous.

Finally, since for all $x \in Q(D), v_{0}(x) \leq v(x) \leq v_{0}(x)+t$ and $v^{\prime}(\phi(x)) \leq v_{0}(x) \leq v^{\prime}(\phi(x))+t$, it follows that $v^{\prime}(\phi(x)) \leq v(x) \leq v^{\prime}(\phi(x))+2 t$.

Recall from Definition 3.2 the growth rate function $\rho^{\prime}$ of $M_{n s}\left(F^{\prime}\right)$ with respect to $v^{\prime}$. Then using Proposition 4.3, we see that for all $x \in \widehat{Q}$ :

$\rho(x)=\lim _{n \rightarrow \infty} \frac{v\left(x^{n}\right)}{n} \leq \lim _{n \rightarrow \infty} \frac{v^{\prime}\left(x^{n}\right)+2 t}{n}=\rho^{\prime}(x) \leq \rho(x)-$ forcing equality.

Therefore $\rho^{\prime}=\rho$ when restricted to $\widehat{Q}$.

\subsection{Diagonalisation}

Again, recall our Mahler expansion (5):

$$
0=q_{1}^{p^{m}} \tau \partial_{1}(y)+\ldots .+q_{d}^{p^{m}} \tau \partial_{d}(y)+O\left(q^{p^{m}}\right)
$$

Where each $q_{i}=\tau\left(u\left(h_{i}\right)-1\right)$ for some basis $\left\{h_{1}, \cdots, h_{d}\right\}$ for $H$, and $\rho(q)>\rho\left(q_{i}\right)$ for each $i$.

We may embed $Q(D)$ continuously into $M_{s}\left(F^{\prime}\right)$ for any finite extension $F^{\prime}$ of $F=Z(Q(D))$ by Proposition 4.3, and since each $q_{i}$ is a square matrix over $Q(D)$, by choosing $F^{\prime}$ appropriately, we may ensure that they can be reduced to Jordan normal form inside $M_{n s}\left(F^{\prime}\right)$.

But since $F^{\prime}$ has characteristic $p$, after raising to sufficiently high $p$ 'th powers, a Jordan block becomes diagonal. So we may choose $m_{0} \in \mathbb{N}$ such that $q_{i}^{p^{m_{0}}}$ is diagonalisable for each $i$.

But $q_{1}, \cdots, q_{d}$ commute, and it is well known that commuting matrices can be simultaneously diagonalised. Hence there exists $a \in M_{n s}\left(F^{\prime}\right)$ invertible such that $a q_{i}^{p^{m_{0}}} a^{-1}$ is diagonal for each $i$.

So, let $t_{i}:=a q_{i} a^{-1}$, then after multiplying (5) on the left by $a$, we get:

$$
0=t_{1}^{p^{m}} a \tau \partial_{1}(y)+\ldots+t_{d}^{p^{m}} a \tau \partial_{d}(y)+O\left(a q^{p^{m}}\right)
$$


Note that since $t_{i}^{p^{m_{0}}}$ is diagonal for each $i, \rho^{\prime}\left(t_{i}^{p^{m_{0}}}\right)=v^{\prime}\left(t_{i}^{p^{m_{0}}}\right)$, and $\rho^{\prime}\left(t_{i}\right)=\rho^{\prime}\left(q_{i}\right)$ since growth rates are invariant under conjugation by Lemma 3.7 (iii). Since $\rho^{\prime}=\rho$ on $\widehat{Q}$, it follows that $\rho\left(q_{i}^{p^{m_{0}}}\right)=v^{\prime}\left(t_{i}^{p^{m_{0}}}\right)$.

Moreover, $v^{\prime}\left(t_{i}^{p^{m}}\right)=\rho\left(q_{i}^{p^{m}}\right)=p^{m} \rho\left(q_{i}\right)$ for each $m \geq m_{0}$.

Also, $q_{i}=\tau\left(u\left(h_{i}\right)-1\right)=\tau\left(u_{0}\left(h_{i}\right)-1\right)^{p^{m_{1}}}$, so after replacing $m_{1}$ by $m_{1}+m_{0}$ we may ensure that each $t_{i}$ is diagonal, and hence $v^{\prime}\left(t_{i}\right)=\rho\left(q_{i}\right)$.

Now, let $K:=\{h \in H: \rho(\tau(u(h)-1))>\lambda\}$. Then since $i d$ is a non-trivial GPP and $K=K_{i d}$, it follows from Lemma 3.9 that $K$ is a proper open subgroup of $H$ containing $H^{p}$.

For the rest of this section, fix a basis $\left\{h_{1}, \cdots, h_{d}\right\}$ for $H$ such that $\left\{h_{1}^{p}, \cdots, h_{r}^{p}, h_{r+1}, \cdots, h_{d}\right\}$ is a basis for $K, q_{i}=\tau\left(u\left(h_{i}\right)-1\right), t_{i}=a q_{i} a^{-1}$ as above.

Then it follows that for all $i \leq r, v^{\prime}\left(t_{i}\right)=\rho\left(q_{i}\right)=\lambda$, and for $i>r, v^{\prime}\left(t_{i}\right)>\lambda$, so we have:

$$
0=t_{1}^{p^{m}} a \tau \partial_{1}(y)+\ldots+t_{r}^{p^{m}} a \tau \partial_{r}(y)+O\left(a q^{p^{m}}\right)
$$

Where $\rho(q)>\lambda$.

Definition 4.1. Given $c_{1}, \cdots, c_{m} \in M_{n s}\left(F^{\prime}\right)$ with $v^{\prime}\left(c_{i}\right)=\mu$ for some $i$, we say that $c_{1}, \cdots, c_{m}$ are $\mathbb{F}_{p^{-}}$ linearly independent modulo $\mu^{+}$if for any $\alpha_{1}, \cdots, \alpha_{m} \in \mathbb{F}_{p}, v^{\prime}\left(\alpha_{1} c_{1}+\cdots+\alpha_{m} c_{m}\right)>\mu$ if and only if $\alpha_{i}=0$ for all $i$.

Lemma 4.4. $t_{1}, \cdots, t_{r}$ are $\mathbb{F}_{p}$-linearly dependent modulo $\lambda^{+}$.

Proof. Suppose, for contradiction, that $v^{\prime}\left(\alpha_{1} t_{1}+\cdots+\alpha_{r} t_{r}\right)>\lambda$ for some $\alpha_{i} \in \mathbb{F}_{p}$, not all zero, then using Lemma 3.7 ( $i$ ii $)$ we see that

$\rho\left(\alpha_{1} q_{1}+\cdots+\alpha_{r} q_{r}\right)=\rho\left(a\left(\alpha_{1} q_{1}+\cdots+\alpha_{r} q_{r}\right) a^{-1}\right)=\rho\left(\alpha_{1} t_{1}+\cdots+\alpha_{r} t_{r}\right)=v^{\prime}\left(\alpha_{1} t_{1}+\cdots+\alpha_{r} t_{r}\right)>\lambda$.

But since $q_{i}=\tau\left(u\left(h_{i}\right)-1\right)$ for each $i$, we can see using expansions in $k G$ that $\alpha_{1} q_{1}+\cdots+\alpha_{r} q_{r}=$ $\tau\left(u\left(h_{1}^{\alpha_{1}} \cdots h_{r}^{\alpha_{r}}\right)-1\right)+O\left(q_{i} q_{j}\right)$, and clearly $\rho\left(O\left(q_{i} q_{j}\right)\right)>\lambda$, and hence $\rho\left(\tau\left(u\left(h_{1}^{\alpha_{1}} \cdots h_{r}^{\alpha_{r}}\right)-1\right)\right)>\lambda$.

But $K=\{g \in G: \rho(\tau(u(g)-1))>\lambda\}=\left\langle h_{1}^{p}, \cdots, h_{r}^{p}, h_{r+1}, \cdots, h_{d}, X\right\rangle$, so since $p$ does not divide every $\alpha_{i}$, it follows that $h_{1}^{\alpha_{1}} \cdots h_{r}^{\alpha_{r}} \notin K$, and hence $\rho\left(\tau\left(u\left(h_{1}^{\alpha_{1}} \cdots h_{r}^{\alpha_{r}}\right)-1\right)\right)=\lambda$ - contradiction.

Notation: For each $i=1, \cdots, n s$, denote by $e_{i}$ the diagonal matrix with 1 in the $i$ 'th diagonal position, 0 elsewhere.

Proposition 4.5. Suppose $d_{1}, \cdots, d_{r} \in M_{n s}\left(F^{\prime}\right)$ are diagonal, $v^{\prime}\left(d_{i}\right)=\lambda$ for each $i$, and suppose that for all $m \in \mathbb{N}$ we have:

$$
0=d_{1}^{p^{m}} a_{1}+\cdots+d_{r}^{p^{m}} a_{r}+O\left(a q^{p^{m}}\right)
$$

Where $a_{i}, a, q \in M_{n s}\left(F^{\prime}\right), \rho(q)>\lambda$.

Suppose further that for some $j \in\{1, \cdots, n s\}$, the $j$ 'th entries of $d_{1}, \cdots, d_{r}$ are $\mathbb{F}_{p}$-linearly independent modulo $\lambda^{+}$. Then $e_{j} a_{i}=0$ for all $i=1, \cdots, r$.

Proof. Firstly, since $d_{1, j}, \cdots, d_{r, j}$ are $\mathbb{F}_{p}$-linearly independent modulo $\lambda^{+}$, it follows immediately that $e_{j} d_{1}, \cdots, e_{j} d_{r}$ are $\mathbb{F}_{p}$-linearly independent modulo $\lambda^{+}$. And:

$$
0=\left(e_{j} d_{1}\right)^{p^{m}} a_{1}+\cdots+\left(e_{j} d_{r}\right)^{p^{m}} a_{r}+O\left(e_{j} a q^{p^{m}}\right)
$$

For convenience, set $d_{i}^{\prime}:=e_{j} d_{i}$, and in a similar vein to the proof of Theorem 3.12 , define the following matrices:

$$
D_{m}:=\left(\begin{array}{ccccc}
d_{1}^{\prime p^{m}} & d_{2}^{p^{m}} & \cdot & \cdot & d_{r}^{p^{m}} \\
d_{1}^{\prime p^{m+1}} & d_{2}^{\prime p^{m+1}} & \cdot & \cdot & d_{r}^{p^{m+1}} \\
\cdot & \cdot & \cdot & \cdot & \cdot \\
\cdot & \cdot & \cdot & \cdot & \cdot \\
d_{1}^{\prime p^{m+t-1}} & d_{2}^{\prime p^{m+t-1}} & \cdot & \cdot & d_{r}^{\prime p^{m+t-1}}
\end{array}\right), \underline{a}:=\left(\begin{array}{c}
a_{1} \\
a_{2} \\
\cdot \\
\cdot \\
\cdot \\
a_{r}
\end{array}\right)
$$

Then we can rewrite our expression as: 


$$
0=D_{m} \underline{a}+\left(\begin{array}{c}
O\left(e_{j} a q^{p^{m}}\right) \\
O\left(e_{j} a q^{p^{m+1}}\right) \\
\cdot \\
\cdot \\
\cdot \\
O\left(e_{j} a q^{p^{m+t-1}}\right)
\end{array}\right)
$$

And multiplying by $\operatorname{adj}\left(D_{m}\right)$ gives:

$$
0=\operatorname{det}\left(D_{m}\right) \underline{a}+\operatorname{adj}\left(D_{m}\right)\left(\begin{array}{c}
O\left(e_{j} q^{p^{m}}\right) \\
O\left(e_{j} a q^{p^{m+1}}\right) \\
\cdot \\
\cdot \\
\cdot \\
O\left(e_{j} a q^{p^{m+t-1}}\right)
\end{array}\right)
$$

And the proof of Lemma 3.10 shows that the $(i, j)$-entry of $\operatorname{adj}\left(D_{m}\right)$ has value at least $\frac{p^{r}-1}{p-1} p^{m} \lambda-p^{m+j-1} \lambda$.

Since $\rho(q)>\lambda$, fix $c>0$ such that $\rho(q)>\lambda+c$, and hence $v^{\prime}\left(e_{j} a q^{p^{m}}\right) \geq p^{m} \lambda+p^{m} c+v(a)$ for all sufficiently high $m$. Then we see that the $i$ 'th entry of the vector

$$
\operatorname{adj}\left(D_{m}\right)\left(\begin{array}{c}
O\left(e_{j} a q^{p^{m}}\right) \\
O\left(e_{j} a q^{p^{m+1}}\right) \\
\cdot \\
\cdot \\
\cdot \\
O\left(e_{j} a q^{p^{m+t-1}}\right)
\end{array}\right)
$$

has value at least $\frac{p^{r}-1}{p-1} p^{m} \lambda+p^{m} c+v(a)$ for $m>>0$.

So examining the $i^{\prime}$ th entry of our expression (10) gives that $0=\operatorname{det}\left(D_{m}\right) a_{i}+\epsilon_{i, m}$, where $v^{\prime}\left(\epsilon_{i, m}\right) \geq$ $\frac{p^{t}-1}{p-1} p^{m+r} \lambda+p^{m} c+v(a)$.

Let $\Delta:=\operatorname{det}\left(D_{0}\right)$, then $\operatorname{det}\left(D_{m}\right)=\Delta^{p^{m}}$ for all $m \in \mathbb{N}$, and using [4, Lemma 1.1(ii)] we see that

$$
\Delta=\beta \cdot \prod_{\alpha \in \mathbb{P}^{r-1} \mathbb{F}_{p}}\left(\alpha_{1} d_{1}^{\prime}+\cdots+\alpha_{r} d_{r}^{\prime}\right) \text { for some } \beta \in \mathbb{F}_{p}
$$

Since $d_{1}^{\prime}, \cdots, d_{r}^{\prime}$ are $\mathbb{F}_{p^{-}}$-linearly independent modulo $\lambda^{+}$, each term in this product has value $\lambda$, and moreover is a diagonal matrix, with only the $j^{\prime}$ th diagonal entry non-zero.

Let $\delta$ be the $j$ 'th diagonal entry of $\Delta$. Then $\delta \in F^{\prime}, \delta^{-1} \Delta=e_{j}$, and $v(\delta)=\sum_{\alpha \in \mathbb{P}^{r-1} \mathbb{F}_{p}} \lambda=\frac{p^{r}-1}{p-1} \lambda$. So:

$$
0=\delta^{-p^{m}} \Delta^{p^{m}} a_{i}+\delta^{-p^{m}} \epsilon_{i, m}=e_{j} a_{i}+\delta^{-p^{m}} \epsilon_{i, m}
$$

and $v^{\prime}\left(\delta^{-p^{m}} \epsilon_{i, m}\right)=v^{\prime}\left(\epsilon_{i, m}\right)-p^{m} \frac{p^{r}-1}{p-1} \lambda \geq \frac{p^{r}-1}{p-1} p^{m} \lambda+p^{m} c+v(a)-\frac{p^{m}-1}{p-1} p^{m} \lambda=v(a)+p^{m} c \rightarrow \infty$ as $m \rightarrow \infty$.

Hence $\delta^{-p^{m}} \epsilon_{i, m} \rightarrow 0$ and $e_{j} a_{i}=0$ as required.

\subsection{Linear Dependence}

Consider again the maps $\partial_{1}, \cdots, \partial_{r}: k G \rightarrow k G$. These are $k$-linear derivations of $k G$, and we want to prove that $\partial_{i}(P)=0$ for all $i$.

Lemma 4.6. Let $\delta: k G \rightarrow k G$ be any $k$-linear derivation of $k G$. Then if $c \tau \delta(P)=0$ for some $0 \neq c \in M_{n s}\left(F^{\prime}\right)$ then $\tau \delta(P)=0$

Proof. Let $I=\left\{a \in M_{n s}\left(F^{\prime}\right): a \tau \delta(P)=0\right\}$, then it is clear that $I$ is a left ideal of $M_{n s}\left(F^{\prime}\right)$, and $I \neq 0$ since $0 \neq c \in I$. We want to prove that $1 \in I$, and hence $\tau \delta(P)=0$.

We will first prove that $I$ is right $\widehat{Q}$-invariant: 
Given $r \in k G, y \in P, \delta(r y)=r \delta(y)+\delta(r) y$ since $\delta$ is a derivation. So $\tau \delta(r y)=\tau(r) \tau \delta(y)+\tau \delta(r) \tau(y)=$ $\tau(r) \tau \delta(y)$.

Therefore, for any $a \in I, a \tau(r) \tau \delta(y)=a \tau \delta(r y)=0$ since $r y \in P$. Thus $a \tau(r) \in I$.

It follows that $I$ is right $\frac{k G}{P}$-invariant.

Given $s \in k G$, regular $\bmod P$ (i.e. $\tau(s)$ is a unit in $\left.Q\left(\frac{k G}{P}\right)\right)$, we have that $I \tau(s) \subseteq I$. Hence we have a descending chain $I \supseteq I \tau(s) \supseteq I \tau(s)^{2} \supseteq \cdots$ of right ideals of $M_{n s}\left(F^{\prime}\right)$.

So since $M_{n s}\left(F^{\prime}\right)$ is artinian, it follows that $I \tau(s)^{n}=I \tau(s)^{n+1}$ for some $n \in \mathbb{N}$, so dividing out by $\tau(s)^{n+1}$ gives that $I \tau(s)^{-1}=I$.

Therefore, $I$ is right $Q\left(\frac{k G}{P}\right)$-invariant, and passing to the completion gives that it is right $\widehat{Q}$-invariant as required.

This means that $I \cap \widehat{Q}$ is a two sided ideal of the simple $\operatorname{ring} \widehat{Q} \cong M_{n}(Q(D))$. We will prove that $I \cap \widehat{Q} \neq 0$, and it will follow that $I \cap \widehat{Q}=\widehat{Q}$ and thus $1 \in I$.

We know that $\widehat{Q} \cong M_{n}(Q(D))$ and $Q(D) \hookrightarrow M_{s}\left(F^{\prime}\right)$. Since $Q(D)$ is a division ring, we must have that $M_{s}\left(F^{\prime}\right)$ is free as a right $Q(D)$-module, so let $\left\{x_{1}, \cdots, x_{t}\right\}$ be a basis for $M_{s}\left(F^{\prime}\right)$ over $Q(D)$.

It follows easily that $\left\{x_{1} I_{n s}, \cdots, x_{t} I_{n s}\right\}$ is a basis for $M_{n s}\left(F^{\prime}\right)$ over $M_{n}(Q(D))=\widehat{Q}$.

Now, $c \in I$ and $c \neq 0$, so $c=x_{1} c_{1}+\cdots+x_{t} c_{t}$ for some $c_{i} \in \widehat{Q}$, not all zero, and $c \tau \delta(y)=0$ for all $y \in P$.

Therefore $0=c \tau \delta(y)=x_{1}\left(c_{1} \tau \delta(y)\right)+x_{2}\left(c_{2} \tau \delta_{2}(y)\right)+\cdots+x_{t}\left(c_{t} \tau \delta(y)\right)$, so it follows from $\widehat{Q}$-linear independence of $x_{1} I_{n s}, \cdots, x_{t} I_{n s}$ that $c_{i} \tau \delta(y)=0$ for all $i$, and hence $c_{i} \in I \cap \widehat{Q}$.

So choose $i$ such that $c_{i} \neq 0$, and since $c_{i} \in I \cap \widehat{Q}$, we have that $I \neq 0$ as required.

Theorem 4.7. Let $\delta_{1}, \cdots, \delta_{r}: k G \rightarrow k G$ be $k$-linear derivations of $k G$, and suppose that there exist matrices $a, q, d_{1}, \cdots, d_{r} \in M_{n s}\left(F^{\prime}\right)$ such that a is invertible, the $d_{i}$ are diagonal of value $\lambda, \rho(q)>\lambda$ and for all $y \in P$ :

$$
0=d_{1}^{p^{m}} a \tau \delta_{1}(y)+d_{2}^{p^{m}} a \tau \delta_{2}(y)+\cdots+d_{r}^{p^{m}} a \tau \delta_{r}(y)+O\left(a q^{p^{m}}\right)
$$

Suppose further that $d_{1}, \cdots, d_{r}$ are $\mathbb{F}_{p}$-linearly independent modulo $\lambda^{+}$, then $\tau \delta_{i}(P)=0$ for all $i$.

Proof. We will use induction on $r$. First suppose that $r=1$.

Then since $0=d_{1}^{p^{m}} a \tau \delta_{1}(y)+O\left(a q^{p^{m}}\right)$, it follows immediately from Proposition 4.5 that $e_{j} a \tau \delta_{1}(y)=0$ for any $j=1, \cdots, n s$ such that $v\left(d_{1, j}\right)=\lambda$, and this holds for all $y \in P$.

Since $a$ is a unit, $e_{j} a \neq 0$, so using Lemma 4.6. we see that $\tau \delta_{1}(P)=0$ as required.

Now suppose, for induction, that the result holds for $r-1$ :

Assume first that there exists $j=1, \cdots, n s$ such that $d_{1, j}, \cdots, d_{r, j}$ are $\mathbb{F}_{p}$-linearly independent modulo $\lambda^{+}$. Then using Proposition 4.5 and Lemma 4.6 again, we see that $e_{j} a \tau \delta_{i}(y)=0$ for all $i=1, \cdots, r, y \in P$, and hence $\tau \delta_{i}(P)=0$ for all $i$ as required.

Hence we may assume that all the corresponding entries of $d_{1}, \cdots, d_{r}$ are $\mathbb{F}_{p}$-linearly dependent modulo $\lambda^{+}$, i.e. given $j=1, \cdots, n s$, we can find $\beta_{1}, \cdots, \beta_{r} \in \mathbb{F}_{p}$ such that $v\left(\beta_{1} d_{1, j}+\cdots+\beta_{r} d_{r, j}\right)>\lambda$. We can of course choose $j$ such that $v\left(d_{i, j}\right)=\lambda$ for some $i$.

Assume without loss of generality that $v\left(d_{r, j}\right)=\lambda$ and that $\beta_{r} \neq 0$, so after rescaling we may assume that $\beta_{r}=-1$.

It follows immediately that $v^{\prime}\left(e_{j} \beta_{1} d_{1}+\cdots+e_{j} \beta_{r-1} d_{r-1}-e_{j} d_{r}\right)>\lambda$, so set $\epsilon:=e_{j} \beta_{1} d_{1, j}+\cdots+e_{j} \beta_{r-1} d_{r-1, j}-e_{j} d_{r, j}$ for convenience.

Multiplying our expression by $e_{j}$ gives:

$0=e_{j} d_{1}^{p^{m}} a \tau \delta_{1}(y)+\cdots+e_{j} d_{r-1}^{p^{m}} a \tau \delta_{r-1}(y)+e_{j} d_{r}^{p^{m}} a \tau \delta_{r}(y)+O\left(e_{j} a q^{p^{m}}\right)$ 


$$
\begin{aligned}
& =e_{j} d_{1}^{p^{m}} a \tau \delta_{1}(y)+\cdots+e_{j} d_{r-1}^{p^{m}} a \tau \delta_{r-1}(y)-e_{j}\left(\beta_{1} d_{1}+\cdots+\beta_{r-1} d_{r-1}\right)^{p^{m}} a \tau \delta_{r}(y)+\epsilon^{p^{m}} a \tau \delta_{r}(y)+O\left(a q^{p^{m}}\right) \\
& =\left(e_{j} d_{1}\right)^{p^{m}} a\left(\tau \delta_{1}-\beta_{1} \tau \delta_{r}\right)(y)+\cdots+\left(e_{j} d_{r-1}\right)^{p^{m}} a\left(\tau \delta_{r-1}-\beta_{r-1} \tau \delta_{r}\right)(y)+\epsilon^{p^{m}} a \tau \delta_{r}(y)+O\left(a q^{p^{m}}\right) .
\end{aligned}
$$

Now, set $\delta_{i}^{\prime}:=\delta_{i}-\beta_{i} \delta_{r}, d_{i}^{\prime}:=e_{j} d_{i}$ for each $i=1, \cdots, r-1$. Then the $\delta_{i}^{\prime}$ are $k$-linear derivations of $k G$, and since $\epsilon$ is diagonal and $v^{\prime}(\epsilon)>\lambda$, it follows that $\rho(\epsilon)>\lambda$, and so $\epsilon^{p^{m}} a \tau \delta_{r}(y)+O\left(a q^{p^{m}}\right)=O\left(a q^{\prime p^{m}}\right)$ for some $q^{\prime}$ with $\rho\left(q^{\prime}\right)>\lambda$. Hence:

$$
0=d_{1}^{\prime p^{m}} a \tau \delta_{1}^{\prime}(y)+d_{2}^{\prime p^{m}} a \tau \delta_{2}^{\prime}(y)+\cdots+d_{r-1}^{p^{m}} a \tau \delta_{r-1}^{\prime}(y)+O\left(a q^{\prime p^{m}}\right)
$$

So it follows from induction that $\tau \delta_{i}^{\prime}(P)=0$ for all $i$, i.e. for all $y \in P, \tau \delta_{i}(y)=\beta_{i} \tau \delta_{r}(y)$, and:

$$
\begin{aligned}
& 0=d_{1}^{p^{m}} a \tau \delta_{1}(y)+\cdots+d_{r}^{p^{m}} a \tau \delta_{r}(y)+O\left(a q^{p^{m}}\right) \\
& =d_{1}^{p^{m}} a\left(\beta_{1} \tau \delta_{r}\right)(y)+\cdots+d_{r-1}^{p^{m}} a\left(\beta_{r-1} \tau \delta_{r}\right)(y)+d_{r}^{p^{m}} a \tau \delta_{r}(y)+O\left(a q^{p^{m}}\right) \\
& =\left(\beta_{1} d_{1}+\beta_{2} d_{2}+\cdots+\beta_{r-1} d_{r-1}+d_{r}\right)^{p^{m}} a \tau \delta_{r}(y)+O\left(a q^{p^{m}}\right) .
\end{aligned}
$$

But since $d_{1}, \cdots, d_{r}$ are $\mathbb{F}_{p^{-}}$-linearly independent modulo $\lambda^{+}$, it follows that $v^{\prime}\left(\beta_{1} q_{1}+\cdots+\beta_{r-1} d_{r-1}+d_{r}\right)=\lambda$, so just applying Proposition 4.5 and Lemma 4.6 again gives that $\tau \delta_{r}(P)=0$.

So, we have $0=d_{1}^{p^{m}} a \tau \delta_{1}(y)+\cdots+d_{r-1}^{p^{m}} a \tau \delta_{r-1}(y)+O\left(a q^{p^{m}}\right)$. Therefore $\tau \delta_{i}(P)=0$ for all $i<r$ by induction.

Corollary 4.8. Let $G$ be a p-valuable, abelian-by-procyclic group, $P \in \operatorname{Spec}^{f}(k G)$ such that $Q\left(\frac{k G}{P}\right)$ is a CSA. Then $P$ is controlled by a proper open subgroup of $G$.

Proof. We know that $t_{1}, \cdots, t_{r}$ are $\mathbb{F}_{p}$-linearly independent modulo $\lambda^{+}$by Lemma 4.4, so applying Theorem 4.7 with $\delta_{i}=\partial_{i}$ and $d_{i}=t_{i}$, it follows that $\tau \partial_{i}(P)=0$ for all $i=1, \cdots, r$.

Hence $P$ is controlled by a proper open subgroup of $G$ by Proposition 2.4 ,

So, from now on, we may assume that $Q\left(\frac{k G}{P}\right)$ is not a CSA.

\section{The Extended Commutator Subgroup}

Again, fix a non-abelian, $p$-valuable, abelian-by-procyclic group $G$, with principal subgroup $H$, procyclic element $X$. We will assume further that $G$ has split-centre, i.e. $1 \rightarrow Z(G) \rightarrow G \rightarrow \frac{G}{Z(G)} \rightarrow 1$ is split exact.

\subsection{Uniform groups}

Assume for now that $G$ is uniform, i.e. that $(G, G) \subseteq G^{p^{\epsilon}}$, where $\epsilon=2$ if $p=2$ and $\epsilon=1$ if $p>2$. Note that uniform group $G$ is $p$-saturable using the $p$-valuation $\omega(g)=\max \left\{n \in \mathbb{N}: g \in G^{p^{n-\epsilon}}\right\}$ (see [7, Chapter 4] for full details).

Recall from [7, Chapter 9], that a free $\mathbb{Z}_{p}$-Lie algebra $\mathfrak{g}$ of finite rank is powerful if $[\mathfrak{g}, \mathfrak{g}] \subseteq p^{\epsilon} \mathfrak{g}$. It follows from [7. Theorem 9.10] that a $p$-saturable group $G$ is uniform if and only if $\log (G)$ is powerful.

Let $\mathfrak{g}=\log (G)$, then using Lie theory, we see that $\mathfrak{g}=\mathfrak{h} \rtimes \operatorname{Span}_{\mathbb{Z}_{p}}\{x\}$, where $\mathfrak{h}=\log (H), x=\log (X)$, and $[\mathfrak{g}, \mathfrak{g}]=[x, \mathfrak{h}] \subseteq p \mathfrak{h}$.

Recall that a map $w: \mathfrak{g} \rightarrow \mathbb{R} \cup\{\infty\}$ is a valuation if for all $u, v \in \mathfrak{g}, \alpha \in \mathbb{Z}_{p}$ :

- $w(u+v) \geq \min \{w(u), w(v)\}$,

- $w([u, v]) \geq w(u)+w(v)$,

- $w(\alpha u)=v_{p}(\alpha)+w(u)$,

- $w(u)=\infty$ if and only if $u=0$,

- $w(u)>\frac{1}{p-1}$.

Also recall from [15, Proposition 32.6] that if $w$ is a valuation on $\mathfrak{g}$, then $w$ corresponds to a $p$-valuation $\omega$ on $G$ defined by $\omega(g):=w(\log (g))$. 
Proposition 5.1. Let $G$ be a non-abelian, uniform, abelian-by-procyclic group with split-centre, let $\mathfrak{g}:=$ $\log (G)$, and let $V:=\exp ([\mathfrak{g}, \mathfrak{g}]) \subseteq H^{p}$. Then there exists a basis $\left\{h_{1}, \cdots, h_{d}\right\}$ for $H, r \leq d$ such that $\left\{h_{r+1}, \cdots, h_{d}\right\}$ is a basis for $Z(G)$ and $\left\{h_{1}^{p^{t_{1}}}, \cdots, h_{r}^{p^{t_{r}}}\right\}$ is a basis for $V$ for some $t_{i} \geq 1$.

Moreover, there exists an abelian p-valuation $\omega$ on $G$ such that $(i)\left\{h_{1}, \cdots, h_{d}, X\right\}$ is an ordered basis for $(G, \omega)$, and $(i i) \omega\left(h_{1}^{p^{t_{1}}}\right)=\omega\left(h_{2}^{p^{t_{2}}}\right)=\cdots=\omega\left(h_{r}^{p^{t_{r}}}\right)>\omega(X)$.

Proof. First, note that since $G$ has split centre, we have that $G \cong Z(G) \times \frac{G}{Z(G)}$. In fact, since $Z(G) \subseteq H$, we have that $H \cong Z(G) \times H^{\prime}$ for some $H^{\prime} \leq H$, normal and isolated in $G$.

It follows that $\mathfrak{h}=Z(\mathfrak{g}) \oplus \mathfrak{h}^{\prime}$, where $\mathfrak{h}^{\prime}:=\log \left(H^{\prime}\right)$, and clearly $[\mathfrak{g}, \mathfrak{g}]=[x, \mathfrak{h}]=\left[x, \mathfrak{h}^{\prime}\right]$.

By the Elementary Divisors Theorem, there exists a basis $\left\{v_{1}, \cdots, v_{r}\right\}$ for $\mathfrak{h}^{\prime}$ such that $\left\{p^{t_{1}} v_{1}, \ldots, p^{t_{r}} v_{r}\right\}$ is a basis for $\left[x, \mathfrak{h}^{\prime}\right]$ for some $t_{i} \geq 0$. And since $\mathfrak{g}$ is powerful, we have in fact that $t_{i} \geq \epsilon$ for each $i$.

Let $\left\{v_{r+1}, \cdots, v_{d}\right\}$ be any basis for $Z(\mathfrak{g})$, and set $h_{i}:=\exp \left(v_{i}\right)$ for each $i=1, \cdots d$. It follows that $\left\{h_{1}^{p^{t_{1}}}, \ldots ., h_{r}^{p^{t_{r}}}\right\}$ is a basis for $V$, and that $\left\{h_{r+1}, \cdots, h_{d}\right\}$ is a basis for $Z(G)$ as required.

Now, the proof of [15, Lemma 26.13] shows that if $\omega$ is any $p$-valuation on $G$ and we choose $c>0$ with $\omega(g)>c+\frac{1}{p-1}$ for all $g \in G$, then $\omega_{c}(g):=\omega(g)-c$ defines a new $p$-valuation on $G$ satisfying $\omega_{c}((g, h))>$ $\omega_{c}(g)+\omega_{c}(h)$, which preserves ordered bases.

So if $\omega$ is an integer valued $p$-valuation satisfying $i$ and $i i$, then take $c:=\frac{1}{e}$ for any integer $e \geq 2$ and $\omega_{c}$ will also satisfy $i$ and $i i$. Also $\omega_{c}(G) \subseteq \frac{1}{e} \mathbb{Z}$ and $\omega_{c}((g, h))>\omega_{c}(g)+\omega_{c}(h)$ for all $g, h \in G$, i.e. $\omega_{c}$ is abelian.

Therefore, it remains to show that we can define an integer valued $p$-valuation on $G$ satisfying $i$ and $i i$.

Assume without loss of generality that $t_{1} \geq t_{i}$ for all $i=1, \cdots, r$. Choose $a \in \mathbb{Z}$ with $a>\epsilon$, and set $a_{i}:=a+t_{1}-t_{i}$ for each $i$, so that $a_{i}+t_{i}=a_{j}+t_{j}$ for all $i, j=1, \cdots, r$.

For convenience, set $v_{d+1}:=x$, and for each $i>r$, set $a_{i}=\epsilon$. Then define:

$$
w: \mathfrak{g} \rightarrow \mathbb{Z} \cup\{\infty\}, \sum_{i=1, \ldots, d} \alpha_{i} v_{i} \mapsto \min \left\{v_{p}\left(\alpha_{i}\right)+a_{i}: i=1, \ldots, d\right\} .
$$

We will prove that $w$ is a valuation on $\mathfrak{g}$, and that $w\left(p^{t_{i}} v_{i}\right)=w\left(p^{t_{j}} v_{j}\right)>w(x)$ for all $i, j \leq r$. Then by defining $\omega$ on $G$ by $\omega(g)=w(\log (g))$, the result will follow.

Firstly, the property that $w\left(p^{t_{i}} u_{i}\right)=w\left(p^{t_{j}} u_{j}\right)>w(x)$ is clear, since $w\left(p^{t_{i}} u_{i}\right)=t_{i}+a_{i}=t_{j}+a_{j}=w\left(p^{t_{j}} u_{j}\right)$ for all $i, j<d$, and $a_{i}+t_{i}=a+t_{1} \geq a>1=w(x)$.

It is also clear from the definition of $w$ that $w(u+v) \geq \min \{w(u), w(v)\}, w(\alpha u)=v_{p}(\alpha)+w(u), w(u)=\infty$ if and only if $u=0$, and $w(u)>\frac{1}{p-1}$ for all $u, v \in \mathfrak{g}, \alpha \in \mathbb{Z}_{p}$.

Therefore it remains to prove that $w([u, v]) \geq w(u)+w(v)$, and it is straightforward to show that it suffices to prove this for basis elements.

So since $v_{r+1}, \cdots, v_{d}$ are central, we need only to show that $w\left(\left[x, v_{i}\right]\right) \geq w(x)+w\left(v_{i}\right)$ for all $i \leq r$.

We have that $\left[x, v_{i}\right]=\alpha_{i, 1} p^{t_{1}} v_{1}+\ldots .+\alpha_{i, r} p^{t_{r}} v_{r}$ for some $\alpha_{i, j} \in \mathbb{Z}_{p}$, so:

$w\left(\left[x, v_{i}\right]\right)=\min _{j=1, \ldots, r}\left\{v_{p}\left(\alpha_{i, j}\right)+t_{j}+a_{j}\right\}=\min _{j=1, \ldots, r}\left\{v_{p}\left(\alpha_{i, j}\right)\right\}+t_{i}+a_{i} \geq a_{i}+t_{i} \geq a_{i}+\epsilon=w\left(v_{i}\right)+w(x)$.

Remark: This result does not hold in general if $G$ is not uniform. For example, if $p>2$ and $\mathfrak{g}=\operatorname{Span}_{\mathbb{Z}_{p}}\{x, y, z\}$ with $[y, z]=0,[x, y]=p y,[x, z]=y+p z$, then $\mathfrak{g}$ is not powerful, and there is no valuation $w$ on $\mathfrak{g}$ that equates the values of basis elements for $[\mathfrak{g}, \mathfrak{g}]$.

Now suppose that $G$ is any non-abelian, $p$-valuable, abelian-by-procyclic group with split centre, principal subgroup $H$.

Lemma 5.2. For any $h \in H, g \in G$, set $v=\log (h), u=\log (g)$, then $(g, h)=\exp \left(\sum_{n \geq 1} \frac{1}{n !}(a d(u))^{n}(v)\right)$ 
Proof. $g h g^{-1}=g \exp (v) g^{-1}=\sum_{n \geq 0} \frac{1}{n !}\left(g v g^{-1}\right)^{n}=\exp \left(g v g^{-1}\right)$.

Let $l_{x}, r_{x}$ be left and right multiplication by $x$, then note that $l_{\exp (x)}=\exp \left(l_{x}\right)$, same for $r_{x}$.

Then $g v g^{-1}=\exp (u) v \exp (u)^{-1}=\exp (u) v \exp (-u)=\left(l_{\exp (u)} r_{\exp (-u)}\right)(v)$

$=\left(\exp \left(l_{u}\right) \exp \left(r_{-u}\right)\right)(v)=\exp \left(l_{u}-r_{u}\right)(v)=\exp (\operatorname{ad}(u))(v)=\sum_{n \geq 0} \frac{1}{n !}(\operatorname{ad}(u))^{n}(v)$.

Therefore $g h g^{-1}=\exp \left(g v g^{-1}\right)=\exp \left(\sum_{n \geq 0} \frac{1}{n !}(a d(u))^{n}(v)\right)$.

Finally, $\log ((g, h))=\log \left(\left(g h g^{-1}\right) h^{-1}\right)=\log \left(g h g^{-1}\right)-\log (h)$ since $h$ and $g h g^{-1}$ commute. Clearly this is equal to $\sum_{n \geq 1} \frac{1}{n !}(a d(u))^{n}(v)$ as required.

We know that $G=H \rtimes\langle X\rangle$, so for each $m \in \mathbb{N}$, define $G_{m}:=H \rtimes\left\langle X^{p^{m}}\right\rangle$, and it is immediate that $G_{m}$ is an open, normal subgroup of $G$, and that it is non-abelian, $p$-valuable, abelian-by-procyclic with principal subgroup $H$, procyclic element $X^{p^{m}}$ and split centre.

Lemma 5.3. There exists $m \in \mathbb{N}$ such that $G_{m}$ is a uniform group.

Proof. Recall that $G$ is an open subgroup of the $p$-saturated group $\operatorname{Sat}(G)$, i.e there exists $t \in \mathbb{N}$ with $\operatorname{Sat}(G)^{p^{t}} \subseteq G$. Choose any such $t$ and let $m:=t+\epsilon$.

Given $h \in H$, by Lemma 5.2 we have that $\left(X^{p^{m}}, h\right)=\exp \left(\sum_{n \geq 1} \frac{1}{n !}\left(\operatorname{ad}\left(p^{m} x\right)^{n}(v)\right)\right.$ where $x=\log (X)$ and $v=\log (h)$ lie in $\log (\operatorname{Sat}(G))$.

We want to prove that $\left(X^{p^{m}}, h\right) \in H^{p^{\epsilon}}=G^{p^{\epsilon}} \cap H$, so since $\operatorname{Sat}(G)^{p^{m}}=\operatorname{Sat}(G)^{p^{t+\epsilon}} \subseteq G^{p^{\epsilon}}$, it suffices to prove that $\frac{1}{n !} a d\left(p^{m} x\right)^{n}(v) \in p^{m} \log (\operatorname{Sat}(G))$ for all $n \geq 1$.

Clearly, for each $n, \operatorname{ad}\left(p^{m} x\right)^{n}(v)=\left[p^{m} x, \operatorname{ad}\left(p^{m} x\right)^{n-1}(u)\right]$, so we only need to prove that $\operatorname{ad}\left(p^{m} x\right)^{n-1}(u) \in$ $p^{v_{p}(n !)} \log (\operatorname{Sat}(G))$, in which case:

$\frac{1}{n !} a d\left(p^{m} x\right)^{n}(v)=\frac{p^{m}}{n !}\left[x, \operatorname{ad}\left(p^{m} x\right)^{n-1}(u)\right] \in \frac{p^{v_{p}(n !)+m}}{n !} \log (\operatorname{Sat}(G)) \subseteq p^{m} \log (\operatorname{Sat}(G))$.

Let $w$ be a saturated valuation on $\log (\operatorname{Sat}(G))$, i.e. if $w(x)>\frac{1}{p-1}+1$ then $x=p y$ for some $y \in \log (\operatorname{Sat}(G))$.

Then since $w\left(a d\left(p^{m} x\right)^{n-1}(u)\right) \geq(n-1) w\left(p^{m} x\right)+w(u)>\frac{n-1}{p-1}+\frac{1}{p-1}$, it follows that $a d\left(p^{m} x\right)^{n-1}(u)=p^{k} v$ for some $v \in \log (\operatorname{Sat}(G)), k \geq \frac{n-1}{p-1}$.

We will show that $k \geq v_{p}(n !)$, and it will follow that $a d\left(p^{m} x\right)^{n-1}(u)=p^{k} v \in p^{v_{p}(n !)} \log (\operatorname{Sat}(G))$.

If $n=a_{0}+a_{1} p+\cdots+a_{r} p^{r}$ for some $0 \leq a_{i}<p$, then let $s(n)=a_{0}+a_{1}+\cdots+a_{r}$. We know from [10, I 2.2.3] that $v_{p}(n !)=\frac{n-s(n)}{p-1}$.

Suppose that $v_{p}(n !)>\frac{n-1}{p-1}$, i.e. $\frac{n-s(n)}{p-1}>\frac{n-1}{p-1}$, and hence $s(n)<1$. This means that $s(n)=0$ and hence $n=0$ - contradiction.

Therefore $k \geq \frac{n-1}{p-1} \geq v_{p}(n !)$ as required.

\subsection{Extension of Filtration}

From now on, fix $c \in \mathbb{N}$ minimal such that $G_{c}$ is uniform, we know that this exists by Lemma 5.3 . Let $\mathfrak{g}:=\log \left(G_{c}\right)$ - a powerful $\mathbb{Z}_{p}$-subalgebra of $\log (\operatorname{Sat}(G))$.

Definition 5.1. Define the extended commutator subgroup of $G$ to be

$$
c(G):=(Z(G) \times \exp ([\mathfrak{g}, \mathfrak{g}])) \rtimes\left\langle X^{p^{c}}\right\rangle \subseteq G_{c} .
$$

Proposition 5.4. If $G$ is any p-valuable, abelian-by-procyclic group with split centre, then:

i. $c(G)$ is an open normal subgroup of $G$. 
ii. There exists a basis $\left\{k_{1}, k_{2}, \cdots, k_{d}\right\}$ for $H$ such that $\left\{k_{r+1}, \cdots, k_{d}\right\}$ is a basis for $Z(G)$ and $\left\{u_{c}\left(k_{1}\right), u_{c}\left(k_{2}\right), \cdots, u_{c}\left(k_{r}\right), k_{r+1}, \cdots, k_{d}, X^{p^{c}}\right\}$ is a basis for $c(G)$.

iii. We may choose this basis $\left\{k_{1}, \cdots, k_{d}\right\}$ such that for each $i \leq r$, there exist $\alpha_{i, j} \in \mathbb{Z}_{p}$ with $p \mid \alpha_{i, j}$ for $j<i$ and $\alpha_{i, i}=1$, such that $X u_{c}\left(k_{i}\right) X^{-1}=u_{c}\left(k_{1}\right)^{\alpha_{i, 1}} \cdots u_{c}\left(k_{d}\right)^{\alpha_{i, d}}$.

iv. There is an abelian p-valuation $\omega$ on $c(G)$ such that $\left\{u_{c}\left(k_{1}\right), \cdots, u_{c}\left(k_{r}\right), k_{r+1}, \cdots, k_{d}, X^{p^{c}}\right\}$ is an ordered

basis for $(c(G), \omega)$ and $\omega\left(u_{c}\left(k_{i}\right)\right)=\omega\left(u_{c}\left(k_{j}\right)\right)>\omega\left(X^{p^{c}}\right)$ for all $i, j \leq r$.

Proof. Let $V=\exp ([\mathfrak{g}, \mathfrak{g}])$, and let $x=\log (X) \in \log (\operatorname{Sat}(G))$.

If $h \in V$ then $h=\exp \left(\left[p^{c} x, u\right]\right)$ for some $u \in \log (H)$, i.e. $u=\log (k)$, and so:

$$
h=\exp \left(\left[\log \left(X^{p^{c}}\right), \log (k)\right]\right)=\lim _{n \rightarrow \infty}\left(X^{p^{n+c}} k^{p^{n}} X^{-p^{n+c}} k^{-p^{n}}\right)^{p^{-2 n}} \text { by [10, IV. 3.2.3]. }
$$

Thus $X h X^{-1}=\lim _{n \rightarrow \infty}\left(X^{p^{n+c}}\left(X k X^{-1}\right)^{p^{n}} X^{-p^{n+c}}\left(X k X^{-1}\right)^{-p^{n}}\right)^{p^{-2 n}}=\exp \left(\left[\log \left(X^{p^{c}}\right), \log \left(X k X^{-1}\right)\right]\right)$.

Clearly this lies in $V$, and hence $V$ is normal in $G$.

Using Lemma 5.2, it is straightforward to show that for all $h \in H,\left(X^{p^{c}}, h\right) \in V$, therefore:

$$
h X^{p^{c}} h^{-1}=\left(X^{p^{c}}, h\right)^{-1} X^{p^{c}} \in V \rtimes\left\langle X^{p^{c}}\right\rangle
$$

It follows that $c(G)=Z(G) \times V \rtimes\left\langle X^{p^{c}}\right\rangle$ is normal in $G$.

Using Proposition 5.1, we may choose a basis $\left\{h_{1}, \cdots, h_{d}\right\}$ for $H$ such that $\left\{h_{r+1}, \cdots, h_{d}\right\}$ is a basis for $Z(G)$ and $\left\{h_{1}^{p^{t_{1}}}, \cdots, h_{r}^{p^{t_{r}}}\right\}$ is a basis for $V$.

Therefore $c(G)$ has basis $\left\{h_{1}^{p^{t_{1}}}, \cdots, h_{r}^{p^{t_{r}}}, h_{r+1}, \cdots, h_{d}, X^{p^{c}}\right\}$, and hence it is open in $G$ as required.

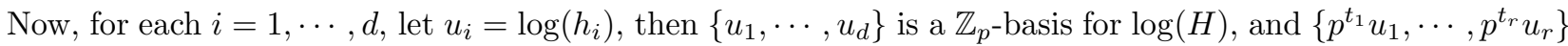
is a basis for $[\mathfrak{g}, \mathfrak{g}]$.

Therefore, for each $i, p^{t_{i}} u_{i}=\left[p^{c} x, v_{i}\right]$ for some $v_{i} \in \log (H)$, in fact we may assume that $v_{i} \in \operatorname{Span}_{\mathbb{Z}_{p}}\left\{u_{1}, \cdots, u_{r}\right\}$, and it follows that $\left\{v_{1}, \cdots, v_{r}\right\}$ forms a basis for $\operatorname{Span}_{\mathbb{Z}_{p}}\left\{u_{1}, \cdots, u_{r}\right\}$.

Let $k_{i}:=\exp \left(v_{i}\right)$ for each $i=1, \cdots r$, and for $i>r$ set $k_{i}=h_{i}$. Then we know that

$$
u_{c}\left(k_{i}\right)=\exp \left(\left[p^{c} \log (X), \log \left(k_{i}\right)\right]\right)=\exp \left(\left[p^{c} x, v_{i}\right]\right)=\exp \left(p^{t_{i}} u_{i}\right)=h_{i}^{p^{t_{i}}} \text { for each } i \leq r
$$

It follows that $\left\{u_{c}\left(k_{1}\right), \cdots, u_{c}\left(k_{r}\right), k_{r+1}, \cdots, k_{d}, X^{p^{c}}\right\}$ is a basis for $c(G)$, thus giving part $i i$.

Now, $V$ is normal in $G$, and clearly $V^{p}$ is also normal, so consider the action $\psi$ of $X$ on the $r$-dimensional

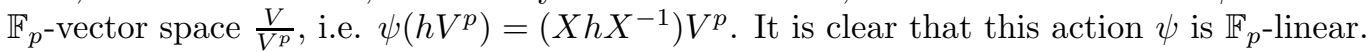

Furthermore, since $G_{c}$ is uniform and $X^{p^{c}} \in G_{c}$, we have that $\psi^{p^{c}}=i d$, i.e. $(\psi-i d)^{p^{c}}=0$. Therefore $\psi$ has a 1-eigenvector in $\frac{V}{V^{p}}$.

It follows that we may choose a basis for $\frac{V}{V^{p}}$ such that $\psi$ is represented by an upper-triangular matrix, with 1 's on the diagonal.

This basis is obtained by transforming $\left\{u_{c}\left(k_{1}\right), \cdots, u_{c}\left(k_{r}\right)\right\}=\left\{h_{1}^{p^{t_{1}}}, \cdots, h_{r}^{p^{t_{r}}}\right\}$ by an invertible matrix over $\mathbb{Z}_{p}$. The new basis will also have the same form $\left\{u_{c}\left(k_{1}^{\prime}\right), \cdots, u_{c}\left(k_{r}^{\prime}\right)\right\}=\left\{h_{1}^{\prime p^{t_{1}}}, \cdots, h_{r}^{p^{t_{r}}}\right\}$ as described by $i i$, and it will satisfy iii as required.

Finally, using Proposition 5.1, we see that there is an abelian $p$-valuation $\omega$ on the uniform group $G_{c}$ such that $\omega\left(u_{c}\left(k_{i}\right)\right)=\omega\left(u_{c}\left(k_{j}\right)\right)>\omega\left(X^{p^{c}}\right)$ for all $i, j \leq r$, and of course $\omega$ restricts to $c(G)$, which gives us part $i v$.

We call a basis $\left\{k_{1}, \cdots, k_{d}\right\}$ for $H$ satisfying these conditions a $k$-basis for $H$.

This result gives us a $p$-valuation that equates the values of $u\left(k_{i}\right)$ and $u\left(k_{j}\right)$ for each $i, j$. Unfortunately, with the standard Lazard filtration, this does not mean that $w\left(u\left(k_{i}\right)-1\right)=w\left(u\left(k_{j}\right)-1\right)$. 
Theorem 5.5. Let $G$ be a non-abelian, p-valuable, abelian-by-procyclic group with split centre. Let $c(G)$ be the extended commutator subgroup, and let $\left\{k_{1}, \cdots, k_{d}\right\}$ be a $k$-basis for $H$.

Then there exists a complete, Zariskian filtration $w: k G \rightarrow \mathbb{Z} \cup\{\infty\}$ such that:

i. For all $i, j=1, \cdots, r, w\left(u_{c}\left(k_{i}\right)-1\right)=w\left(u_{c}\left(k_{j}\right)-1\right)=\theta$ for some integer $\theta>0$.

ii. The associated graded $g r k G \cong k\left[T_{1}, \cdots, T_{d+1}\right] * \frac{G}{c(G)}$, where $T_{i}=\operatorname{gr}\left(u_{c}\left(k_{i}\right)-1\right)$ for $i \leq r, T_{i}=\operatorname{gr}\left(k_{i}-1\right)$ for $r+1 \leq i \leq d$ and $T_{d+1}=\operatorname{gr}\left(X^{p^{c}}-1\right)$. Each $T_{i}$ has positive degree, and $\operatorname{deg}\left(T_{i}\right)=\theta$ for $i=1, \cdots, r$.

iii. Set $\bar{X}:=\operatorname{gr}(X)$. Then $T_{r}$ is central, and for each $i<r, \bar{X} T_{i} \bar{X}^{-1}=T_{i}+D_{i}$ for some $D_{i} \in$ $\operatorname{Span}_{\mathbb{F}_{p}}\left\{T_{i+1}, \cdots, T_{r}\right\}$.

Let $A:=\left(k\left[T_{1}, \cdots, T_{d+1}\right]\right)^{\frac{G}{c(G)}}$ be the ring of invariants, then $A$ is Noetherian, central in gr $k G$, and $k\left[T_{1}, \cdots, T_{d+1}\right]$ is finitely generated over $A$.

Proof. Set $U=c(G)=\left\langle u_{c}\left(k_{1}\right), \cdots, u_{c}\left(k_{r}\right), k_{r+1}, \cdots, k_{d}, X^{p^{c}}\right\rangle$. Then $U$ is an open, normal subgroup of $G$ by Proposition $5.4(i)$, and hence $k G \cong k U * \frac{G}{U}$.

Using Proposition $5.4(i v)$, we choose an abelian $p$-valuation $\omega$ on $U$ such that $\frac{1}{e} \theta:=\omega\left(u_{c}\left(k_{i}\right)\right)=\omega\left(u_{c}\left(k_{j}\right)\right)>$ $\omega\left(X^{p^{c}}\right)$ for all $i, j \leq r$, where $\theta>0$ is an integer. Then we can define the Lazard valuation $w$ on $k U$ with respect to $\omega$.

Since $\left\{u_{c}\left(k_{1}\right), \cdots, u_{c}\left(k_{r}\right), k_{r+1}, \cdots, k_{d}, X^{p^{c}}\right\}$ is an ordered basis for $(U, \omega)$, it follows from the definition of $w$ that:

$$
w\left(u_{c}\left(k_{j}\right)-1\right)=e \omega\left(u_{c}\left(k_{j}\right)\right)=e \omega\left(u_{c}\left(k_{i}\right)\right)=w\left(u_{c}\left(k_{i}\right)-1\right)=\theta \text { for all } i, j \leq r .
$$

Furthermore, we have that if $V=\exp ([\mathfrak{g}, \mathfrak{g}]) \subseteq U$ and $r \in k V$, then $w(r) \geq \theta$.

We want to apply Proposition 2.2 and extend $w$ to $k G \cong k U * \frac{G}{U}$. So we only need to verify that for all $g \in G$, $r \in k U, w\left(g r g^{-1}\right)=w(r)$, and it suffices to verify this property for $r=u_{c}\left(k_{1}\right)-1, \cdots, u_{c}\left(k_{r}\right)-1$,

$k_{r+1}-1, \cdots, k_{d}-1, X^{p^{c}}-1$, since they form a topological generating set for $k U$.

Since $k_{r+1}, \cdots, k_{d} \in Z(G)$, it is obvious that $w\left(g\left(k_{l}-1\right) g^{-1}\right)=w\left(k_{l}-1\right)$ for each $r+1 \leq l \leq d, g \in G$.

For each $j \leq r, g u_{c}\left(k_{j}\right) g^{-1} \in V$, thus:

$w\left(g u_{c}\left(k_{j}\right) g^{-1}-1\right) \geq \theta=w\left(u_{c}\left(k_{j}\right)-1\right)$ and it follows easily that equality holds.

Finally, $g=h X^{\beta}$ for some $h \in H, \beta \in \mathbb{Z}_{p}$, so

$$
g X^{p^{c}} g^{-1}-1=h X^{p^{c}} h^{-1}-1=\left(\left(h, X^{p^{c}}\right)-1\right)\left(X^{p^{c}}-1\right)+\left(\left(h, X^{p^{c}}\right)-1\right)+\left(X^{p^{c}}-1\right) .
$$

Hence $w\left(g\left(X^{p^{c}}-1\right) g^{-1}\right) \geq \min \left\{w\left(\left(h, X^{p^{c}}\right)-1\right), w\left(X^{p^{c}}-1\right)\right\}$, with equality if $w\left(\left(h, X^{p^{c}}\right)-1\right) \neq w\left(X^{p^{c}}-1\right)$. But since $\left(h, X^{p^{c}}\right) \in V$, we have that

$$
w\left(\left(h, X^{p^{c}}\right)-1\right) \geq \theta=e \omega\left(u_{c}\left(k_{i}\right)\right)>e \omega\left(X^{p^{c}}\right)=w\left(X^{p^{c}}-1\right)
$$

and hence $w\left(g X^{p^{c}} g^{-1}-1\right)=w\left(X^{p^{c}}-1\right)$ as required. Note that it is here that we need the fact that $\omega\left(u_{c}\left(k_{i}\right)\right)>\omega\left(X^{p^{c}}\right)$.

Therefore we can apply Proposition 2.2 , and extend $w$ to $k G$ so that $\operatorname{gr}_{w} k G \cong\left(\operatorname{gr}_{w} k U\right) * \frac{G}{U}$, and we have that $\operatorname{gr}_{w} k U \cong k\left[T_{1}, \ldots, T_{d+1}\right]$ as usual, where $T_{i}=\operatorname{gr}\left(u_{c}\left(k_{i}\right)-1\right)$ has degree $\theta$ for $i \leq r, T_{i}=\operatorname{gr}\left(k_{i}-1\right)$ for $r+1 \leq i \leq d$ and $T_{d+1}=\operatorname{gr}\left(X^{p^{c}}-1\right)$ as required.

Using Proposition 5.4 $($ iii $)$, we see that $X u_{c}\left(k_{i}\right) X^{-1}=u_{c}\left(k_{1}\right)^{\alpha_{1, i}} \cdots u_{c}\left(k_{r}\right)^{\alpha_{1, i}}$ where $p \mid \alpha_{j}$ for $j<i$ and $\alpha_{i, i}=1$.

Hence $\bar{X} T_{i} \bar{X}^{-1}=\bar{\alpha}_{i, 1} T_{1}+\cdots+\bar{\alpha}_{i, r} T_{r}=T_{i}+\bar{\alpha}_{i+1,1} T_{i+1}+\cdots+\bar{\alpha}_{i, r} T_{r}$ for each $i \leq r$, thus giving part $i i i$.

Now, every element $u \in$ gr $k U$ is a root of the polynomial $\prod_{g \in \frac{G}{c(G)}}\left(s-g u g^{-1}\right) \in A[s]$, hence gr $k U$ is integral over $A$. 
So since $k \subseteq A$ and gr $k U \cong k\left[T_{1}, \ldots, T_{d+1}\right]$ is a finitely generated $k$-algebra, we have that gr $k U$ is a finitely generated $A$-algebra, and hence finitely generated as an $A$-module by the integral property.

So it follows that $\operatorname{gr}_{w} k G$ is finitely generated as a right $A$-module. Furthermore, since gr $k U$ is Noetherian and commutative, it follows from [8, Theorem 2] that $A$ is Noetherian.

Furthermore, it is easy to show that the twist $\frac{G}{U} \times \frac{G}{U} \rightarrow(\text { gr } k U)^{\times}$of the crossed product is trivial, so it follows that if $r \in \operatorname{gr} k U$ is invariant under the action of $\frac{G}{U}$ then it is central. Hence $A$ is central in gr $k G$.

Note: For any $h \in H$, we have that $\left(u_{c}(h)-1\right)+F_{\theta+1} k G \in \operatorname{Span}_{\mathbb{F}_{p}}\left\{T_{1}, \cdots, T_{r}\right\}$.

Now, let $P$ be a faithful prime ideal of $k G$, and let $w$ be a filtration on $k G$ satisfying the conditions of the proposition. Then $w$ induces the quotient filtration $\bar{w}$ of $\frac{k G}{P}$.

By [12, Ch.II Corollary 2.1.5], $P$ is closed in $k G$, and hence $\frac{k G}{P}$ is complete, and $\operatorname{gr}_{\bar{w}} \frac{k G}{P} \cong \frac{\operatorname{gr} P}{\operatorname{gr} P}$ is Noetherian. Therefore $\bar{w}$ is Zariskian by [12, Ch.II Theorem 2.1.2], and clearly $\tau: k G \rightarrow \frac{k G}{P}$ is continuous.

For convenience, set $\bar{T}:=T+\operatorname{gr} P \in \operatorname{gr} \frac{k G}{P}$ for all $T \in \operatorname{gr} k c(G)=k\left[T_{1}, \cdots, T_{d+1}\right]$.

Let $\bar{A}:=\frac{A+\operatorname{gr} P}{\operatorname{gr} P}$ be the image of $A$ in gr $\frac{k G}{P}$, and let $A^{\prime}:=\left(k\left[\bar{T}_{1}, \cdots, \bar{T}_{d+1}\right]\right)^{\frac{G}{c(G)}}$ be the ring of $\frac{G}{c(G)}$-invariants in $k\left[\bar{T}_{1}, \cdots, \bar{T}_{d+1}\right]=\frac{k\left[T_{1}, \cdots, T_{d+1}\right]+\mathrm{gr} P}{\mathrm{gr} P}$.

Since $\frac{G}{c(G)}$-invariant elements in gr $k G$ are $\frac{G}{c(G)}$-invariant modulo gr $P$, it is clear that $\bar{A} \subseteq A^{\prime} \subseteq \frac{k\left[T_{1}, \cdots, T_{d+1}\right]+\operatorname{gr} P}{\operatorname{gr} P}$.

Then since $k\left[T_{1}, \cdots, T_{d+1}\right]$ is finitely generated over $A$ by Theorem [5.5] it follows that $A^{\prime}$ is finitely generated over the Noetherian ring $\bar{A}$, hence $A^{\prime}$ is Noetherian.

Therefore, $\frac{k G}{P}$ is a prime ring with a Zariskian filtration $\bar{w}$ such that gr $\frac{k G}{P}$ is finitely generated over a central, Noetherian subring $A^{\prime}$. Hence we may apply Theorem 3.3 to produce a non-commutative valuation on $Q\left(\frac{k G}{P}\right)$.

\subsection{A special case}

In the next section, we will prove Theorem 1.5 in full generality, but first we need to deal with a special case:

Fix a $k$-basis $\left\{k_{1}, \cdots, k_{s}\right\}$ for $H$, and a Zariskian filtration $w$ on $k G$ satisfying the conditions of Theorem 5.5 Then we have that $T_{r} \in A$ and $\bar{X} T_{i} \bar{X}^{-1}=T_{i}+D_{i}$ for some $D_{i} \in \operatorname{Span}_{\mathbb{F}_{p}}\left\{T_{i+1}, \cdots, T_{r}\right\}$ for all $i<r$.

We will now suppose that for each $i<r, D_{i}$ is nilpotent modulo gr $P$.

Then for sufficiently high $m, \bar{X} T_{i}^{p^{m}} \bar{X}^{-1} \equiv\left(T_{i}+D_{i}\right)^{p^{m}}=T_{i}^{p^{m}}+D_{i}^{p^{m}} \equiv T_{i}^{p^{m}}(\bmod \operatorname{gr} P)$, i.e. $\bar{T}_{i}^{p^{m}} \in A^{\prime}$

Fix an integer $m_{0}$ such that $\bar{T}_{i}^{p^{m_{0}}} \in A^{\prime}$ for all $i \leq r$.

Proposition 5.6. Suppose that for each $i=1, \cdots, r, T_{i}$ is nilpotent modulo gr $P$, i.e. $\bar{T}_{i}$ is nilpotent. Then $Q\left(\frac{k G}{P}\right)$ is a central simple algebra.

Proof. Using Theorem[5.5(ii), every element of gr $k G$ has the form

$$
\sum_{g \in \frac{G}{c(G)}}\left(\sum_{\alpha \in \mathbb{N}^{d+1}} \lambda_{\alpha} T_{1}^{\alpha_{1}} \cdots T_{d+1}^{\alpha_{d+1}}\right) g
$$

where $\lambda_{\alpha}=0$ for all but finitely many $\alpha$.

Therefore, it follows immediately from nilpotence of $\bar{T}_{1}, \cdots, \bar{T}_{r}$ that $\frac{\operatorname{gr} k G}{\operatorname{gr} P}$ is finitely generated over $\frac{k\left[T_{r+1}, \cdots, T_{d+1}\right]+\operatorname{gr} P}{\operatorname{gr} P}$.

But since $Z(G)=\left\langle k_{r+1}, \cdots, k_{d}\right\rangle$ by Proposition 5.4 (ii), it follows that under the quotient filtration, gr $\frac{k\left(Z(G) \times\left\langle X^{p^{c}}\right\rangle\right)+P}{P} \cong \frac{k\left[T_{r+1}, \cdots, T_{d+1}\right]+\operatorname{gr} P}{\operatorname{gr} P}$.

So since gr $\frac{k G}{P}$ is finitely generated over gr $\frac{k\left(Z(G) \times\left\langle X^{p^{c}}\right\rangle\right)+P}{P}$, and $\frac{k\left(Z(G) \times\left\langle X^{p^{c}}\right\rangle\right)+P}{P}$ is closed in $\frac{k G}{P}$, it follows from [12, Ch.I Theorem 5.7] that $\frac{k G}{P}$ is finitely generated over $\frac{k\left(Z(G) \times\left\langle X^{p^{c}}\right\rangle\right)+P}{P}$. 
But $k\left(Z(G) \times\left\langle X^{p^{c}}\right\rangle\right)$ is commutative, so $\frac{k G}{P}$ is finitely generated as a right module over a commutative subring. Therefore, by [13, Corollary 13.1.14(iii)], $\frac{k G}{P}$ satisfies a polynomial identity.

So since $\frac{k G}{P}$ is prime, it follows from Posner's theorem [13, Theorem 13.6.5], that $Q\left(\frac{k G}{P}\right)$ is a central simple algebra.

Note: This proof relies on the split centre property, without which we would not be able to argue that $\frac{k\left[T_{r+1}, \cdots, T_{d+1}\right]+\operatorname{gr} P}{\operatorname{gr} P}$ arises as the associated graded of some commutative subring of $\frac{k G}{P}$.

So let us assume that $Q\left(\frac{k G}{P}\right)$ is not a CSA. Then by the proposition, we know that there exists $s \leq r$ such that $\bar{T}_{s}$ is not nilpotent.

Since we know that $\bar{T}_{s}^{p^{m_{0}}} \in A^{\prime}$, it follows there exists a minimal prime ideal $\mathfrak{q}$ of $A^{\prime}$ such that $\bar{T}_{s}^{p^{m_{0}}} \notin \mathfrak{q}$. Using Theorem 3.3 , we let $v=v_{\mathfrak{q}}$ be the non-commutative valuation on $Q\left(\frac{k G}{P}\right)$ corresponding to $\mathfrak{q}$.

So, $\bar{T}_{s}^{p^{m_{0}}}=\operatorname{gr} \tau\left(u_{c}\left(k_{s}\right)-1\right)^{p^{m_{0}}} \in A^{\prime} \backslash \mathfrak{q}$, and hence using Theorem 3.6, we see that $\tau\left(u_{c}\left(k_{s}\right)-1\right)^{p^{k}}$ is $v$-regular for some $k \geq m_{0}$, and hence $\rho\left(\tau\left(u_{c}\left(k_{s}\right)-1\right)^{p^{k}}\right)=v\left(\tau\left(u_{c}\left(k_{s}\right)-1\right)^{p^{k}}\right)$.

Recall that $\lambda=\inf \{\rho(\tau(u(g)-1)): g \in G\}<\infty$ by Lemma 3.8.

Lemma 5.7. Let $h \in H$ such that $\rho(\tau(u(h)-1))=\lambda$. Then $\tau(u(h)-1)^{p^{m}}$ is v-regular for sufficiently high $m$.

Proof. It is clear that $w\left(u_{c}(h)-1\right) \geq \theta=w\left(u_{c}\left(k_{s}\right)-1\right)$, so let $T(h):=u_{c}(h)-1+F_{\theta+1} k G \in \operatorname{Span}_{\mathbb{F}_{p}}\left\{T_{1}, \cdots, T_{r}\right\}$. Then $T(h)=\operatorname{gr}\left(u_{c}(h)-1\right)$ if and only if $w\left(u_{c}(h)-1\right)=\theta$, otherwise $T(h)=0$.

We know that $\operatorname{gr}\left(u_{c}\left(k_{s}\right)-1\right)=T_{s} \notin$ gr $P$, and hence $\bar{w}\left(\tau\left(u_{c}\left(k_{s}\right)-1\right)\right)=w\left(u_{c}\left(k_{s}\right)-1\right)$, giving that $\bar{w}\left(\tau\left(u_{c}(h)-1\right)\right) \geq w\left(u_{c}(h)-1\right) \geq w\left(u_{c}\left(k_{s}\right)-1\right)=\bar{w}\left(\tau\left(u_{c}\left(k_{s}\right)-1\right)\right)$.

Also, $T(h)^{p^{m_{0}}}+\operatorname{gr} P \in A^{\prime}$, and if $T(h)^{p^{m_{0}}}+\operatorname{gr} P \notin \mathfrak{q}$ then $T(h)^{p^{m_{0}}}+\operatorname{gr} P=\operatorname{gr}_{\bar{w}}\left(\tau\left(u_{c}(h)-1\right)^{p^{m_{0}}}\right) \in A^{\prime} \backslash \mathfrak{q}$, so it follows from Theorem 3.6 that $\tau(u(h)-1)^{p^{m}}$ is $v$-regular for $m>>0$.

So, suppose for contradiction that $T(h)^{p^{m_{0}}}+\operatorname{gr} P \in \mathfrak{q}$ :

If $T(h)^{p^{m_{0}}}+\operatorname{gr} P=0$ then $\bar{w}\left(\tau\left(u_{c}(h)-1\right)^{p^{m_{0}}}\right)>p^{m_{0}} \theta=\bar{w}\left(\tau\left(u_{c}\left(k_{s}\right)-1\right)\right)$, and if $T(h)^{p^{m_{0}}} \neq 0$ then $T(h)^{p^{m_{0}}}+$ $\operatorname{gr} P=\operatorname{gr}_{\bar{w}}\left(\tau\left(u_{c}(h)-1\right)^{p^{m_{0}}}\right) \in \mathfrak{q}$. In either case, using Theorem [3.6, it follows that for $m$ sufficiently high:

$$
v\left(\tau\left(u_{c}(h)-1\right)^{p^{m}}\right)>v\left(\tau\left(u_{c}\left(k_{s}\right)-1\right)^{p^{m}}\right) .
$$

Therefore, $v(\tau(u(h)-1))>v\left(\tau\left(u\left(k_{s}\right)-1\right)\right)$, so since $\tau\left(u\left(k_{s}\right)-1\right)^{p^{k}}$ is $v$-regular:

$\rho(\tau(u(h)-1)) \geq \frac{1}{p^{k}} v\left(\tau(u(h)-1)^{p^{k}}\right)>\frac{1}{p^{k}} v\left(\tau\left(u\left(k_{s}\right)-1\right)^{p^{k}}\right)=\rho\left(\tau\left(u\left(k_{s}\right)-1\right)\right) \geq \lambda-$ contradiction.

Recall the definition of a growth preserving polynomial (GPP) from Section 2.4, and recall that the identity map is a non-trivial GPP.

Proposition 5.8. Suppose that $Q\left(\frac{k G}{P}\right)$ is not a CSA, and that $D_{i}$ is nilpotent mod gr $P$ for all $i<r$. Then $i d: \tau(k H) \rightarrow \tau(k H)$ is a special GPP with respect to some non-commutative valuation on $Q\left(\frac{k G}{P}\right)$.

Proof. This is immediate from Definition 3.3 and Lemma 5.7

Therefore, we will assume from now on that $D_{i}$ is not nilpotent $\bmod \operatorname{gr} P$ for some $i$.

Remark: If $G$ is uniform then $D_{i}=0$ for all $i$, but in general we cannot assume this. For example, if $p>2$ and $G=\langle X, Y, Z\rangle$ where $Y$ and $Z$ commute, $X Y X^{-1}=Y^{r}, X Z X^{-1}=(Y Z)^{r}, r=e^{p} \in \mathbb{Z}_{p}$, then $G$ is non-uniform, $c=1$, and $\left\{Z, Y^{p-1} Z^{p}\right\}$ is a $k$-basis for $H=\langle Y, Z\rangle$. In this case, $\bar{X} T_{2} \bar{X}^{-1}=T_{2}$, $\bar{X} T_{1} \bar{X}^{-1}=T_{1}+T_{2}$. 


\section{Construction of Growth Preserving Polynomials}

As in the previous section, we will take $G$ to be a $p$-valuable, abelian-by-procyclic group with split centre, $P$ a faithful prime ideal of $k G$, and $w$ a Zariskian filtration on $k G$ satisfying the conditions of Theorem 5.5 .

We fix a $k$-basis $\left\{k_{1}, \cdots, k_{d}\right\}$ for $H$, and let $T_{i}:=\operatorname{gr}\left(u_{c}\left(k_{i}\right)-1\right)$ for each $i \leq r, T_{i}:=\operatorname{gr}\left(k_{i}-1\right)$ for $i>r$. We know that $T_{r}, \cdots, T_{d}$ are central, and $D_{i}:=\bar{X} T_{i} \bar{X}^{-1}-T_{i} \in \operatorname{Span}_{\mathbb{F}_{p}}\left\{T_{i+1}, \cdots, T_{r}\right\}$ for each $i<r$.

We now assume that not all the $D_{i}$ are nilpotent modulo gr $P$, so let $s<r$ be maximal such that $D_{s}$ is not nilpotent, i.e. for all $i>s, \bar{T}_{i}^{p^{m}} \in A^{\prime}$ for sufficiently high $m$.

Throughout, we will fix $m_{0}$ such that $\bar{T}_{i}^{p^{m_{0}}} \in A^{\prime}$ for all $i>s$, and we may assume that $m_{1} \geq m_{0}$.

By definition, we know that $D_{s} \in \operatorname{Span}_{\mathbb{F}_{p}}\left\{T_{s+1}, \cdots, T_{r}\right\}$, and hence $\bar{D}_{s}^{p^{m_{0}}} \in A^{\prime}$. So since $D_{s}$ is not nilpotent mod gr $P$, we can fix a minimal prime ideal $\mathfrak{q}$ of $A^{\prime}$ such that $\bar{D}_{s}^{p^{m_{0}}} \in A^{\prime} \backslash \mathfrak{q}$.

Let $v=v_{\mathfrak{q}}$ be the corresponding non-commutative valuation given by Theorem 3.3 .

\subsection{The Reduction Coefficients}

Define a function $L$ of commuting variables $x$ and $y$ by:

$$
L(x, y):=x^{p}-x y^{p-1}
$$

Moreover, for commuting variables $x, y_{1}, y_{2}, \cdots, y_{n}$, define the iterated function

$$
L^{(n)}\left(x, y_{1}, y_{2}, \cdots, y_{n}\right):=L\left(L\left(L\left(\cdots\left(L\left(x, y_{1}\right), y_{2}\right), \cdots\right), y_{n}\right)\right.
$$

For $n=0$, we define $L^{(n)}\left(x, y_{1}, \cdots, y_{n}\right):=x$.

We can readily see that for any commutative $\mathbb{F}_{p}$-algebra $S, y_{1}, \cdots, y_{n} \in S, L\left(-, y_{1}, \cdots, y_{n}\right)$ is $\mathbb{F}_{p}$-linear.

Lemma 6.1. Let $S$ be an $\mathbb{F}_{p}$-algebra, and let $y_{1}, \cdots, y_{n} \in S$ commute. Then there exist $a_{0}, a_{1}, \cdots, a_{n-1} \in S$ such that $L^{(n)}\left(x, y_{1}, \cdots, y_{n}\right)=a_{0} x+a_{1} x^{p}+\cdots+a_{n-1} x^{p^{n-1}}+x^{p^{n}}$ for all $x$ commuting with $y_{1}, \cdots, y_{n}$.

Proof. Both statements are trivially true for $n=0$, so assume that they hold for some $n \geq 0$ and proceed by induction on $n$ :

So $L^{(n)}\left(x, y_{1}, \cdots, y_{n}\right)=a_{0} x+a_{1} x^{p}+\cdots+a_{n-1} x^{p^{n-1}}+x^{p^{n}}$, and:

$L^{(n+1)}\left(x, y_{1}, \cdots, y_{n+1}\right)=L^{(n)}\left(x, y_{1}, \cdots, y_{n}\right)^{p}-L^{(n)}\left(x, y_{1}, \cdots, y_{n}\right) y_{n+1}^{p-1}$

$=\left(a_{0} x+\cdots+a_{n-1} x^{p^{n-1}}+x^{p^{n}}\right)^{p}-\left(a_{0} x+\cdots+a_{n-1} x^{p^{n-1}}+x^{p^{n}}\right) y_{n+1}^{p-1}$

$=\left(-a_{0} y_{n+1}^{p-1}\right) x+\left(a_{0}^{p}-a_{1} y_{n+1}^{p-1}\right) x^{p}+\cdots+\left(a_{n-2}^{p}-a_{n-1} y_{n+1}^{p-1}\right) x^{p^{n-1}}+\left(a_{n-1}^{p}-y_{n-1}^{p-1}\right) x^{p^{n}}+x^{p^{n+1}}$.

So setting $b_{0}=\left(-a_{0} y_{n+1}^{p-1}\right), b_{i}=\left(a_{i-1}^{p}-a_{i} y_{n+1}^{p-1}\right)$ for $1 \leq i \leq n\left(\operatorname{taking} a_{n}:=1\right)$, we have that $L^{(n+1)}\left(x, y_{1}, \cdots, y_{n+1}\right)=$ $b_{0} x+b_{1} x^{p}+\cdots+b_{n} x^{p^{n}}+x^{p^{n+1}}$ as required.

Now, let $B_{s}:=D_{s}$, and for each $1 \leq i<s$, let $B_{i}:=L^{(s-i)}\left(D_{i}, B_{s}, \cdots, B_{i+1}\right)$.

Lemma 6.2. For each $i \leq s, L^{(s-i+1)}\left(\bar{T}_{i}, \bar{B}_{s}, \cdots, \bar{B}_{i}\right)^{p^{m_{0}}} \in A^{\prime}$, so in particular $\bar{B}_{i}^{p^{m_{0}}} \in A^{\prime}$.

Proof. Note that $C \in k\left[T_{1}, \cdots, T_{d}\right]$ is central if and only if it is invariant under the action of $\bar{X}$.

Also, $L(C, C)=C^{p}-C C^{p-1}=0$, and if $D$ is $\bar{X}$-invariant, then $\bar{X} L(C, D) \bar{X}^{-1}=L\left(\bar{X} C \bar{X}^{-1}, D\right)$

Notation: Let $Y^{\prime}:=\bar{Y}^{p^{m_{0}}}$ for any $Y \in \operatorname{gr} k G$.

We will proceed by downwards induction on $i$, starting with $i=s$. Clearly $B_{s}^{\prime}=D_{s}^{\prime} \in \operatorname{Span}_{\mathbb{F}_{p}}\left\{T_{s+1}^{\prime}, \cdots, T_{r}^{\prime}\right\}$ is invariant under the action of $\bar{X}$, so: 
$\bar{X} L\left(T_{s}^{\prime}, B_{s}^{\prime}\right) \bar{X}^{-1}=L\left(\bar{X} T_{s}^{\prime} \bar{X}^{-1}, B_{s}^{\prime}\right)=L\left(T_{s}^{\prime}+B_{s}^{\prime}, B_{s}^{\prime}\right)=L\left(T_{s}^{\prime}, B_{s}^{\prime}\right)+L\left(B_{s}, B_{s}\right)=L\left(T_{s}^{\prime}, B_{s}^{\prime}\right)$.

Therefore $L\left(T_{s}^{\prime}, B_{s}^{\prime}\right)$ is $\bar{X}$-invariant and the result holds.

Suppose we have the result for all $s \geq j>i$.

Then $B_{i}^{\prime}=L^{(s-i)}\left(D_{i}^{\prime}, B_{s}^{\prime}, \cdots, B_{i+1}^{\prime}\right)$, and $D_{i}^{\prime} \in \operatorname{Span}_{\mathbb{F}_{p}}\left\{T_{i+1}^{\prime}, \cdots, T_{r}^{\prime}\right\}$.

Using linearity of $L(-, y)$ we have that

$$
B_{i}^{\prime} \in \operatorname{Span}_{\mathbb{F}_{p}}\left\{L^{(s-i)}\left(T_{j}^{\prime}, B_{s}^{\prime}, \cdots, B_{i+1}^{\prime}\right): j=i+1, \cdots, r\right\}
$$

therefore $B_{i}^{\prime}$ is $\bar{X}$-invariant by the inductive hypothesis.

Also, since $B_{s}^{\prime}, \cdots, B_{i+1}^{\prime}$ are $\bar{X}$-invariant, we have that:

$$
\begin{aligned}
& \bar{X} L^{(s-i)}\left(T_{i}^{\prime}, B_{s}^{\prime}, \cdots, B_{i+1}^{\prime}\right) \bar{X}^{-1}=L^{(s-i)}\left(\bar{X} T_{i}^{\prime} \bar{X}^{-1}, B_{s}^{\prime}, \cdots, B_{i+1}^{\prime}\right) \\
& =L^{(s-i)}\left(T_{i}^{\prime}+\bar{X} T_{i}^{\prime} \bar{X}^{-1}-T_{i}^{\prime}, B_{s}^{\prime}, \cdots, B_{i+1}^{\prime}\right)=L^{(s-i)}\left(T_{i}^{\prime}, B_{s}^{\prime}, \cdots, B_{i+1}^{\prime}\right)+B_{i}
\end{aligned}
$$

The final equality follows from linearity of $L\left(-, B_{s}^{\prime}, \cdots, B_{i+1}^{\prime}\right)$ and the fact that $D_{i}^{\prime}=\bar{X} T_{i}^{\prime} \bar{X}^{-1}-T_{i}^{\prime}$.

Set $C:=L^{(s-i)}\left(T_{i}^{\prime}, B_{s}^{\prime}, \cdots, B_{i+1}^{\prime}\right)$, so that

$$
L^{(s-i+1)}\left(T_{i}^{\prime}, B_{s}^{\prime}, \cdots, B_{i}^{\prime}\right)=L\left(A, B_{i}^{\prime}\right)=C^{p}-C B_{i}^{\prime p-1} \text {, and } \bar{X} C \bar{X}^{-1}=C+B_{i}^{\prime}
$$

Then:

$\bar{X} L\left(C, B_{i}^{\prime}\right) \bar{X}^{-1}=L\left(\bar{X} C \bar{X}^{-1}, B_{i}^{\prime}\right)=L\left(C+B_{i}^{\prime}, B_{i}^{\prime}\right)=L\left(C, B_{i}^{\prime}\right)+L\left(B_{i}^{\prime}, B_{i}^{\prime}\right)=L\left(C, B_{i}^{\prime}\right)$

Hence $L\left(C, B_{i}^{\prime}\right)=L^{(s-i+1)}\left(T_{i}^{\prime}, B_{s}^{\prime}, \cdots, B_{i}^{\prime}\right)$ is $\bar{X}$-invariant as required.

It follows immediately from this Lemma that $L^{(s)}\left(\bar{T}, \bar{B}_{s}, \cdots, \bar{B}_{1}\right)^{p^{m_{0}}} \in A^{\prime}$ for all $T \in \operatorname{Span}_{\mathbb{F}_{p}}\left\{T_{1}, \cdots, T_{r}\right\}$ (i.e. for all $\left.T=u_{c}(h)-1+F_{\theta+1} k G\right)$.

Now, for each $i \leq s, D_{i} \in \operatorname{Span}_{\mathbb{F}_{p}}\left\{T_{i+1}, \cdots, T_{r}\right\}$, so either $D_{i}=0$ or $D_{i}=u_{c}\left(f_{i}\right)-1+F_{\theta+1} k G$ for some $f_{i} \in H$ with $w\left(u_{c}\left(f_{i}\right)-1\right)=\theta$.

Definition 6.1. Define $y_{s}:=u_{c}\left(f_{s}\right)-1$, and for each $1 \leq i<s$, define $y_{i} \in k H$ inductively by:

$y_{i}:= \begin{cases}L^{(s-i)}\left(u_{c}\left(f_{i}\right)-1, y_{s}, \cdots, y_{i+1}\right) & D_{i} \neq 0 \\ 0 & D_{i}=0\end{cases}$

And define $b_{i}:=\tau\left(y_{i}\right)^{p^{m_{1}-c}} \in Q\left(\frac{k G}{P}\right)$, we call these $b_{i}$ the reduction coefficients.

For convenience, we will replace $m_{1}$ by $m_{1}+c$, so that $\tau(u(g)-1)=\tau\left(u_{c}(g)-1\right)^{p^{m_{1}}}$ for all $g \in G$, and $b_{i}=\tau\left(y_{i}\right)^{p^{m_{1}}}$. Since $B_{s} \notin \operatorname{gr} P$, it is clear that $\operatorname{gr}_{\bar{w}}\left(b_{s}\right)=\bar{B}_{s}^{p^{m_{1}}}$.

Since $\operatorname{gr}_{\bar{w}}\left(b_{s}\right)=\bar{B}_{s}^{p^{m_{1}}} \in A^{\prime} \backslash \mathfrak{q}$, it follows from Theorem 3.6 that $b_{s}^{p^{k}}$ is $v$-regular for some $k \in \mathbb{N}$. After replacing $m_{1}$ by $m_{1}+k$, we may also assume that $b_{s}$ is $v$-regular.

Lemma 6.3. For all $h \in H, i \leq s$, let $t:=u_{c}(h)-1, T:=t+F_{\theta+1} k G \in$ gr $k G$.

Then $w\left(L^{(s-i)}\left(t, y_{s}, \cdots, y_{i+1}\right)\right) \geq p^{s-i} \theta$, with equality if and only if $\operatorname{gr}\left(L^{(s-i)}\left(t, y_{s}, \cdots, y_{i+1}\right)\right)=L^{(s-i)}\left(T, B_{s}, \cdots B_{i+1}\right)$, otherwise $L^{(s-i)}\left(T, B_{s}, \cdots B_{i+1}\right)=0$.

In particular, for $y_{i} \neq 0, w\left(y_{i}\right) \geq p^{s-i} \theta$, with equality if and only if $B_{i}=\operatorname{gr}\left(y_{i}\right)$, otherwise $B_{i}=0$.

Proof. We will use downwards induction on $i$, with $i=s$ as the base case:

Since $i=s, L^{(s-i)}\left(t, y_{s}, \cdots, y_{i+1}\right)=t$, and clearly $w(t) \geq \theta=p^{s-s} \theta$, and equality holds if and only if $\operatorname{gr}(t)=T$, and otherwise $T=0$ as required. 
Now suppose the result holds for some $i \leq s$, so let $c:=L^{(s-i)}\left(t, y_{s}, \cdots, y_{i+1}\right), C:=L^{(s-i)}\left(T, B_{s}, \cdots, B_{i+1}\right)$. Then by induction, $w(c) \geq p^{s-i} \theta$, with equality if and only if $C=\operatorname{gr}(c)$, and $w\left(y_{i}\right) \geq p^{s-i} \theta$.

So $w\left(L^{(s-i+1)}\left(t, y_{s}, \cdots, y_{i}\right)\right)=w\left(c^{p}-c y_{i}^{p-1}\right) \geq \min \left\{w\left(c^{p}\right), w\left(c y_{i}^{p-1}\right)\right\} \geq \min \left\{p w(c), w(c)+(p-1) w\left(y_{s}\right)\right\} \geq$ $p^{s-i+1} \theta$ as required.

In particular, this argument shows that if $w(c)>p^{s-i} \theta$ then $w\left(c^{p}-c y_{i}^{p-1}\right)>p^{s-i+1} \theta$.

Therefore, if $w\left(L^{(s-i+1)}\left(t, y_{s}, \cdots, y_{i}\right)\right)=p^{s-i+1} \theta$ then $w(c)=p^{s-i} \theta$, and so $C=\operatorname{gr}(c)=c+F_{p^{s-i} \theta} k G$ by induction. In this case, $\operatorname{gr}\left(L^{(s-i+1)}\left(t, y_{s}, \cdots, y_{i}\right)\right)=\left(c^{p}-c y_{i}^{p-1}\right)+F_{p^{s-i+1} \theta_{\theta}} k G$.

Also, since $c, y_{i} \in k c(G)$ and $w$ is a valuation on $k c(G)$, we have that $w\left(c^{p}\right)=p w(c)=p^{s-i+1} \theta$ and $w\left(c y_{i}^{p-1}\right)=w(c)+(p-1) w\left(y_{i}\right) \geq p^{s-i+1} \theta$.

If $w\left(y_{i}\right)>p^{s-i} \theta$ then $c^{p}-c y_{i}^{p-1}+F_{\theta+1} k G=c^{p}+F_{\theta+1} k G=C^{p}$. But since $B_{i}=0$ by induction, this means that $c^{p}-c y_{i}^{p-1}+F_{p^{s-i+1} \theta+1}=C^{p}-C B_{i}^{p-1}$.

Whereas, if $w\left(y_{i}\right)=p^{s-i} \theta$ then $B_{i}=\operatorname{gr}\left(y_{i}\right)=y_{i}+F_{p^{s-i} \theta+1} k G$ by assumption, so $c^{p}-c y_{i}^{p-1}+F_{p^{s-i+1} \theta+1} k G=$ $C^{p}-C B_{i}^{p-1}$ as required.

Finally, if $w\left(L^{(s-i+1)}\left(t, y_{s}, \cdots, y_{i}\right)\right)>p^{s-i+1} \theta$ then $w\left(c^{p}-c y_{i}^{p-1}\right)>p^{s-i+1} \theta$. Clearly if $C=0$ then $L^{(s-i+1)}\left(T, B_{s}, \cdots, B_{i}\right)=C^{p}-C B_{i}^{p-1}=0$, so we may assume that $C \neq 0$, and hence $C=\operatorname{gr}(c)=$ $c+F_{p^{s-i} \theta+1} k G$.

So since $w\left(c^{p}-c y_{i}^{p-1}\right)>p^{s-i+1} \theta$, it follows that $C^{p}-C B_{i}^{p-1}=0$ as required.

Using this Lemma, we see that $\bar{w}\left(b_{i}\right) \geq p^{s-i} \theta$, with equality if and only if $\operatorname{gr} \bar{w}\left(b_{i}\right)=\bar{B}_{i}^{p^{m_{1}}}$.

Notation: For each $0 \leq i \leq s, q \in Q\left(\frac{k G}{P}\right)$ commuting with $b_{1}, \cdots, b_{s}$, define $L_{i}(q):=L^{(s-i)}\left(q, b_{s}, \cdots, b_{i+1}\right)$. e.g. $L_{s}(q)=q, L_{s-1}(q)=q^{p}-q b_{s}^{p-1}, L_{s-2}(q)=q^{p^{2}}-q^{p}\left(b_{s}^{p^{2}-p}-b_{s-1}^{p-1}\right)+q b_{s}^{p-1} b_{s-1}^{p-1}$.

\subsection{Polynomials}

Again, we have $\lambda=\inf \{\rho(\tau(u(g)-1)): g \in G\}<\infty$.

Lemma 6.4. For each $i \leq s, \rho\left(b_{i}\right) \geq p^{s-i} \lambda$, and it follows that $\rho\left(L_{i}(\tau(u(h)-1)) \geq p^{s-i} \lambda\right.$ for all $h \in H$. Moreover, if $\rho\left(b_{i}\right)=p^{s-i} \lambda$ then $b_{i}^{p^{m}}$ is v-regular for $m$ sufficiently high.

Proof. For $i=s$ the result is clear, because $b_{s}=\tau\left(u\left(f_{s}\right)-1\right)$, so $\rho\left(b_{s}\right) \geq \lambda=p^{s-s} \lambda$ by definition. So we will proceed again by downwards induction on $i$.

The inductive hypothesis states that $\rho\left(b_{i+1}\right) \geq p^{s-i-1} \lambda$, and $\rho\left(L_{i+1}(\tau(u(h)-1))\right) \geq p^{s-i-1} \lambda$ for all $h \in H$.

Thus $\rho\left(L_{i}(\tau(u(h)-1))\right)=\rho\left(L_{i+1}(\tau(u(h)-1))^{p}-L_{i+1}(\tau(u(h)-1)) b_{i+1}^{p-1}\right)$

$\geq \min \left\{p \cdot p^{s-i-1} \lambda, p^{s-i-1} \lambda+(p-1) p^{s-i-1} \lambda\right\}=p^{s-i} \lambda$ for all $h$.

By definition, $b_{i}=L^{(s-i)}\left(\tau\left(u\left(f_{i}\right)-1\right), b_{s}, \cdots, b_{i+1}\right)=L_{i}\left(\tau\left(u\left(f_{i}\right)-1\right)\right)$, so

$$
\rho\left(b_{i}\right)=\rho\left(L_{i}\left(\tau\left(u\left(f_{i}\right)-1\right)\right)\right) \geq p^{s-i} \lambda, \text { and the first statement follows. }
$$

Finally, suppose that $\rho\left(b_{i}\right)=p^{s-i} \lambda$ :

Then if $\bar{w}\left(b_{i}\right)>p^{s-i+m_{1}} \theta=\bar{w}\left(b_{s}^{p^{s-i}}\right)$, then $v\left(b_{i}^{p^{m}}\right)>v\left(b_{s}^{p^{s-i+m}}\right)$ for $m>>0$ by Theorem 3.6. So using $v$-regularity of $b_{s}$, we see that $\rho\left(b_{i}\right)>\rho\left(b_{s}^{p^{s-i}}\right) \geq p^{s-i} \lambda-$ contradiction.

Therefore, by Lemma 6.3, we see that $\bar{w}\left(b_{i}\right)=p^{s-i} \theta$ and $\operatorname{gr}_{\bar{w}}\left(b_{i}\right)=\bar{B}_{i}^{p^{m_{1}}}$.

We know that $\bar{B}_{i}^{p^{m_{1}}} \in A^{\prime}$, so suppose that $\bar{B}_{i}^{p^{m_{1}}} \in \mathfrak{q}$. Then since $\bar{w}\left(b_{i}\right)=p^{s-i} \theta=\bar{w}\left(b_{s}^{p^{s-i}}\right)$, it follows again from Theorem 3.6 that $v\left(b_{i}^{p^{m}}\right)>v\left(b_{s}^{p^{s-i+m}}\right)$ for $m>>0$, and hence $\rho\left(b_{i}\right)>p^{s-i} \rho\left(b_{s}\right) \geq p^{s-i} \lambda-$ contradiction.

Hence $\bar{B}_{i}^{p^{m_{0}}}=\operatorname{gr} \bar{w}\left(b_{i}\right) \in A^{\prime} \backslash \mathfrak{q}$, so $b_{i}^{p^{k}}$ is $v$-regular for some $k \in \mathbb{N}$ by Theorem 3.6 . 
Now, using Lemma 6.1, we see that $L_{i}(x)=L^{(s-i)}\left(x, b_{s}, \cdots, b_{i+1}\right)=a_{0} x+a_{1} x^{p}+\cdots+a_{s-i-1} x^{p^{s-i-1}}+x^{p^{s-i}}$ for some $a_{j} \in \tau(k H)$.

Proposition 6.5. For each $i \leq s, L_{i}$ is a growth preserving polynomial of p-degree $s-i$, and $L_{s}$ is not trivial.

Proof. Firstly, it is clear that $L_{s}=i d$, and so $L_{s}$ is a non-trivial GPP.

We first want to prove that for all $q \in \tau(k H)$, if $\rho(q) \geq \lambda$ then $\rho\left(L_{i}(q)\right) \geq p^{s-i} \lambda$, with strict inequality if $\rho(q)>\lambda$. We know that this holds for $i=s$, so as in the proof of Lemma 6.4 we will use downwards induction on $i$.

So suppose that $\rho\left(L_{i+1}(q)\right) \geq p^{s-i-1} \lambda$, with strict inequality if $\rho(q)>\lambda$. Then:

$L_{i}(q)=L_{i+1}(q)^{p}-L_{i+1}(q) b_{i+1}^{p-1}$, so $\rho\left(L_{i}(q)\right) \geq \min \left\{\rho\left(L_{i+1}(q)^{p}\right), \rho\left(L_{i+1}(q) b_{i+1}^{p-1}\right)\right\}$.

But $\rho\left(L_{i+1}(q)^{p}\right) \geq p \cdot p^{s-i-1} \lambda=p^{s-i} \lambda$, and since $\rho\left(b_{i+1}\right) \geq p^{s-i} \lambda$ by Lemma 6.4 $\rho\left(L_{i+1}(q) b_{i+1}^{p-1}\right) \geq$ $\rho\left(L_{i+1}(q)\right)+(p-1) \rho\left(b_{i+1}\right) \geq p^{s-j-1} \lambda+(p-1) p^{s-i-1} \lambda=p^{s-i} \lambda$.

By the inductive hypothesis, both these inequalities are strict if $\rho(q)>\lambda$, and thus $L_{i}$ is a GPP as required.

So all that remains is to prove that one of the $L_{i}$ is special.

\subsection{Trivial growth preserving polynomials}

Let us first suppose that for some $j, L_{j}$ is trivial, i.e. $L_{j}(\tau(u(h)-1))>p^{s-j} \lambda$ for all $h \in H$.

We know that $L_{s}$ is not trivial, so we can fix $j \leq s$ such that $L_{j}$ is non-trivial and $L_{j-1}$ is trivial. We will need the following results:

Lemma 6.6. Let $A$ be a $k$-algebra, with filtration $w$ such that $A$ is complete with respect to $w$. Suppose a $\in A$ and $w\left(a^{p}-a\right)>0$, then $a^{p^{m}} \rightarrow b \in A$ with $b^{p}=b$ as $m \rightarrow \infty$.

Proof. Let $\varepsilon:=a^{p}-a$, then $w(\varepsilon)>0, a$ commutes with $\varepsilon$, and $a^{p}=a+\varepsilon$.

Therefore, since $\operatorname{char}(k)=p, a^{p^{2}}=a^{p}+\varepsilon^{p}=a+\varepsilon+\varepsilon^{p}$, and it follows from induction that for all $m \in \mathbb{N}$, $a^{p^{m+1}}=a+\varepsilon+\varepsilon^{p}+\cdots+\varepsilon^{p^{m}}$.

But $\varepsilon^{p^{m}} \rightarrow 0$ as $m \rightarrow \infty$ since $w(\varepsilon)>0$, so since $A$ is complete, the sum $\sum_{m \geq 0} \varepsilon^{p^{m}}$ converges in $A$, and hence $a^{p^{m}} \rightarrow a+\sum_{m \geq 0} \varepsilon^{p^{m}} \in A$.

So let $b:=a+\sum_{m \geq 0} \varepsilon^{p^{m}}$, then $b^{p}=a^{p}+\left(\sum_{m \geq 0} \varepsilon^{p^{m}}\right)^{p}=a+\varepsilon+\sum_{m \geq 1} \varepsilon^{p^{m}}=a+\sum_{m \geq 0} \varepsilon^{p^{m}}=b$ as required.

Proposition 6.7. Let $Q=\widehat{Q\left(\frac{k G}{P}\right)}$, and let $\delta_{1}, \cdots, \delta_{r}: k G \rightarrow k G$ be derivations such that $\tau \delta_{i}(P) \neq 0$ for all i. Set $N:=\left\{\left(a_{1}, \cdots, a_{r}\right) \in Q^{r}:\left(a_{1} \tau \delta_{1}+\cdots+a_{r} \tau \delta_{r}\right)(P)=0\right\}$.

Then $N$ is a $Q$-bisubmodule of $Q^{r}$, and either $N=0$ or there exist $\alpha_{1}, \cdots, \alpha_{r} \in Z(Q)$, not all zero, such that for all $\left(a_{1}, \cdots, a_{r}\right) \in N, \alpha_{1} a_{1}+\cdots+\alpha_{r} a_{r}=0$.

Proof. Since $v$ is a non-commutative valuation, we have that $Q$ is simple and artinian, and the proof that $N$ is a $Q$-bisubmodule of $Q^{r}$ is similar to the proof of Lemma 4.6. For the second statement, we will proceed using induction on $r$.

First suppose that $r=1$, then $N$ is a two sided ideal of the simple ring $Q$, so it is either 0 or $Q$. But if $N=Q$ then $1 \in Q$ so $\tau \delta_{1}(P)=0$ - contradiction. Hence $N=0$.

Now suppose that the result holds for $r-1$ for some $r>1$. If $N \neq 0$ then there exists $\left(a_{1}, \cdots, a_{r}\right) \in N$ with $a_{i} \neq 0$ for some $i$, and we may assume without loss of generality that $i=1$.

So, let $A:=\left\{a \in Q:\left(a, a_{2}, \cdots, a_{r}\right) \in N\right.$ for some $\left.a_{i} \in Q\right\}$, then clearly $A$ is a two-sided ideal of $Q$, so $A=0$ or $A=Q$. But $A \neq 0$ since $a_{1} \in A$ and $a_{1} \neq 0$. 
Therefore $A=Q$, and hence we have that for all $b \in Q,\left(b, b_{2}, \cdots, b_{r}\right) \in N$ for some $b_{i} \in Q$.

Let $N^{\prime}=\left\{\left(a_{2}, \cdots, a_{r}\right) \in Q^{r-1}:\left(a_{2} \tau \delta_{2}+\cdots+a_{r} \tau \delta_{r}\right)(P)=0\right\}$. Suppose first that $N^{\prime}=0$.

Then if for some $q \in Q,\left(q, x_{2}, \cdots, x_{r}\right),\left(q, x_{2}^{\prime}, \cdots, x_{r}^{\prime}\right) \in N$ for $x_{i}, x_{i}^{\prime} \in Q$, we have that $\left(x_{2}-x_{2}^{\prime}, \cdots, x_{r}-x_{r}^{\prime}\right) \in$ $N^{\prime}=0$, and hence $x_{i}=x_{i}^{\prime}$ for all $i$.

Hence there is a unique $\left(1, \beta_{2}, \cdots, \beta_{r}\right) \in N$.

Given $x \in Q,\left(x, x \beta_{2}, \cdots, x \beta_{r}\right),\left(x, \beta_{2} x, \cdots, \beta_{r} x\right) \in N$, and so $\left(\left[x, \beta_{2}\right], \cdots,\left[x, \beta_{r}\right]\right) \in N^{\prime}$. Hence $\left[x, \beta_{i}\right]=0$ for all $i$, so $\beta_{i} \in Z(Q)$.

Moreover, if $\left(a_{1}, \cdots, a_{r}\right) \in N$, then since $\left(a_{1}, a_{1} \beta_{2}, \cdots, a_{1} \beta_{r}\right) \in N$, it follows that $a_{i}=\beta_{i} a_{1}$ for all $i>1$, and since $\tau \delta_{1}(P) \neq 0$, it is clear that $\beta_{i} \neq 0$ for some $i$, thus giving the result.

So from now on, we may assume that $N^{\prime} \neq 0$, so by the inductive hypothesis, this means that there exist $\alpha_{2}, \cdots, \alpha_{r} \in Z(Q)$, not all zero, such that for all $\left(a_{2}, \cdots, a_{r}\right) \in N^{\prime}, \alpha_{2} a_{2}+\cdots+\alpha_{r} a_{r}=0$.

Again, suppose we have that $\left(a, x_{2}, \cdots, x_{r}\right),\left(a, x_{2}^{\prime}, \cdots, x_{r}^{\prime}\right) \in N$ for some $a, x_{i}, x_{i}^{\prime} \in Q$. Then clearly $\left(x_{2}-x_{2}^{\prime}, \cdots, x_{r}-x_{r}^{\prime}\right) \in N^{\prime}$, and hence $\alpha_{2}\left(x_{2}-x_{2}^{\prime}\right)+\cdots+\alpha_{r}\left(x_{r}-x_{r}^{\prime}\right)=0$, i.e. $\alpha_{2} x_{2}+\cdots+\alpha_{r} x_{r}=$ $\alpha_{2} x_{2}^{\prime}+\cdots+\alpha_{r} x_{r}^{\prime}$.

So, given $q \in Q,\left(1, x_{2}, \cdots, x_{r}\right) \in N$, we have that $\left(q, q x_{2}, \cdots, q x_{r}\right),\left(q, x_{2} q, \cdots, x_{r} q\right) \in N$, and hence

$$
\alpha_{2} q x_{2}+\cdots+\alpha_{r} q x_{r}=\alpha_{2} x_{2} q+\cdots+\alpha_{r} x_{r} q
$$

i.e $\left[q, \alpha_{2} x_{2}+\cdots+\alpha_{r} x_{r}\right]=0$.

Since this holds for all $q \in Q$, it follows that $\alpha_{2} x_{2}+\cdots+\alpha_{r} x_{r} \in Z(Q)$, so let $-\alpha_{1}$ be this value.

In fact, for any $\operatorname{such}\left(1, x_{2}^{\prime}, \cdots, x_{r}^{\prime}\right) \in N, \alpha_{2} x_{2}^{\prime}+\cdots+\alpha_{r} x_{r}^{\prime}=\alpha_{2} x_{2}+\cdots+\alpha_{r} x_{r}=-\alpha_{1}$, so $-\alpha_{1} \in Z(Q)$ is unchanged, regardless of our choice of $x_{i}$.

Finally, suppose that $\left(a_{1}, \cdots, a_{r}\right),\left(1, x_{2}, \cdots, x_{r}\right) \in N$, then $\left(a_{1}, a_{1} x_{2}, \cdots, a_{1} x_{r}\right) \in N$, and hence $\left(a_{2}-\right.$ $\left.a_{1} x_{2}, \cdots, a_{r}-a_{1} x_{r}\right) \in N^{\prime}$. Thus $\alpha_{2}\left(a_{2}-a_{1} x_{2}\right)+\cdots+\alpha_{r}\left(a_{r}-a_{1} x_{r}\right)=0$, i.e.

$$
\alpha_{2} a_{2}+\cdots+\alpha_{r} a_{r}=a_{1}\left(\alpha_{2} x_{2}+\cdots+\alpha_{r} x_{r}\right)=-\alpha_{1} a_{1}
$$

Therefore $\alpha_{1} a_{1}+\alpha_{2} a_{2}+\cdots+\alpha_{r} a_{r}=0$, and $\alpha_{i} \in Z(Q)$ as required.

Recall from Lemma 3.9 that for any GPP $f$ of $p$-degree $r, K_{f}:=\left\{h \in H: \rho(f(\tau(u(h)-1)))>p^{r} \lambda\right\}$ is an open subgroup of $H$ containing $H^{p}$, and that it is proper in $H$ if $f$ is non-trivial. For each $i \leq s$, define $K_{i}:=K_{L_{i}}$.

Then since $L_{j-1}$ is trivial and $L_{j}$ is not, we know that $K_{j-1}=H$ and $K_{j}$ is a proper subgroup of $H$.

Lemma 6.8. There exists $k \in \mathbb{N}$ such that $b_{j}^{p^{k}}$ is v-regular of value $p^{k+s-j} \lambda$, and for any $h \in H \backslash K_{j}$, $\left(L_{j}\left(\tau(u(h)-1) b_{j}^{-1}\right)^{p^{m}} \rightarrow c \in \widehat{Q\left(\frac{k G}{P}\right)}\right.$ with $c \neq 0$ and $c^{p}=c$.

Proof. Since $L_{j-1}$ is trivial, we know that for each $h \in H, \rho\left(L_{j-1}(\tau(u(h)-1))\right)>p^{s-j+1} \lambda$.

Choose $h \in H \backslash K_{j}$, i.e. $\rho\left(L_{j}(\tau(u(h)-1))=p^{s-j} \lambda\right.$. Setting $q:=\tau(u(h)-1)$ for convenience, we have:

$$
\rho\left(L_{j-1}(q)\right)=\rho\left(L_{j}(q)^{p}-L_{j}(q) b_{j}^{p-1}\right)>p^{s-j+1} \lambda
$$

But $\rho\left(L_{j}(q)^{p}-L_{j}(q) b_{j}^{p-1}\right) \geq \min \left\{\rho\left(L_{j}(q)^{p}\right), \rho\left(L_{j}(q) b_{j}^{p-1}\right)\right\}$, with equality if $\rho\left(L_{j}(q)^{p}\right) \neq \rho\left(L_{j}(q) b_{j}^{p-1}\right)$.

So if $\rho\left(b_{j}\right)>p^{s-j} \lambda$, then we have that:

$$
\rho\left(L_{j}(q) b_{j}^{p-1}\right)>\rho\left(L_{j}(q)\right)+(p-1) p^{s-j} \lambda=p^{s-j} \lambda
$$


But $\rho\left(L_{j}(q)^{p}\right)=p \rho\left(L_{j}(q)\right)=p^{s-j+1} \lambda$, and hence $\rho\left(L_{j-1}(\tau(u(h)-1))\right)=\min \left\{\rho\left(L_{j}(q)^{p}\right), \rho\left(L_{j}(q) b_{j}^{p-1}\right)\right\}$ $=p^{s-j+1} \lambda-$ contradiction.

Therefore, $\rho\left(b_{j}\right) \leq p^{s-j} \lambda$, so using Lemma 6.4, we see that $\rho\left(b_{j}\right)=p^{s-j} \lambda$, and $b_{j}^{p^{k}}$ is $v$-regular for some $k$, and thus $v\left(b_{j}^{p^{k}}\right)=\rho\left(b_{j}^{p^{k}}\right)=p^{k+s-j} \lambda$.

Now, $\rho\left(\left(L_{j}(q)^{p^{k}} b_{j}^{-p^{k}}\right)^{p}-\left(L_{j}(q)^{p^{k}} b_{j}^{-p^{k}}\right)\right)=\rho\left(b_{j}^{-p^{k+1}}\left(L_{j}(q)^{p}-L_{j}(q) b_{j}^{p-1}\right)^{p^{k}}\right)$

$=\rho\left(L_{j-1}(q)^{p^{k}}\right)-p v\left(b_{j}^{p^{k}}\right)>p^{s-j+k+1} \lambda-p^{s-j+k+1} \lambda=0$

This means that $v\left(\left(\left(L_{j}(q) b_{j}^{-1}\right)^{p}-\left(L_{j}(q) b_{j}^{-1}\right)\right)^{p^{m}}\right)>0$ for $m>>0$, so it follows from Lemma 6.6 that $\left.L_{j}(\tau(u(h)-1)) b_{j}^{-1}\right)^{p^{m}}$ converges to $c \in \widehat{Q\left(\frac{k G}{P}\right)}$ with $c^{p}=c$.

Finally, since $\rho\left(L_{j}(\tau(u(h)-1))^{p^{k}} b_{j}^{-p^{k}}\right)=0$, it follows that $c \neq 0$.

Theorem 6.9. If $L_{j-1}$ is trivial and $L_{j}$ is not trivial, then $P$ is controlled by a proper open subgroup of $G$.

Proof. Since $K_{j}$ is a proper subgroup of $H$ containing $H^{p}$, we can choose an ordered basis $\left\{h_{1}, \cdots, h_{d}\right\}$ for $H$ such that $\left\{h_{1}^{p}, \cdots, h_{t}^{p}, h_{t+1}, \cdots, h_{d}\right\}$ is an ordered basis for $K_{j}$.

Consider our Mahler expression (6), taking $f=L_{j}, q_{i}=\tau\left(u\left(h_{i}\right)-1\right)$ :

$$
0=L_{j}\left(q_{1}\right)^{p^{m}} \tau \partial_{1}(y)+\cdots+L_{j}\left(q_{d}\right)^{p^{m}} \tau \partial_{d}(y)+O\left(L_{j}(q)^{p^{m}}\right)
$$

Where $\rho(q)>\lambda$, and hence $\rho\left(L_{j}(q)\right)>p^{s-j} \lambda$. Note that we also have:

$$
\rho\left(L_{j}\left(q_{i}\right)\right)=p^{s-j} \lambda \text { for all } i \leq t \text {, and } \rho\left(L_{j}\left(q_{i}\right)\right)>p^{s-j} \lambda \text { for all } i>t .
$$

Using Lemma 6.8, we see that $b_{j}^{p^{k}}$ is $v$-regular of value $p^{k+s-j}$ for some $k$, and for each $i \leq t,\left(L_{j}\left(q_{i}\right)^{p^{k}} b_{j}^{-p^{k}}\right)^{p^{m}} \rightarrow$ $c_{i} \neq 0$ as $m \rightarrow \infty$, with $c_{i}^{p}=c_{i}$. Clearly $c_{1}, \cdots, c_{r}$ commute.

So, divide out our expression (13) by $b_{j}^{p^{m}}$, which is $v$-regular of value $p^{m+s-j} \lambda$ to obtain:

$$
0=\left(b_{j}^{-1} L_{j}\left(q_{1}\right)\right)^{p^{m}} \tau \partial_{1}(y)+\cdots+\left(b_{j}^{-1} L_{j}\left(q_{d-1}\right)\right)^{p^{m}} \tau \partial_{d-1}(y)+O\left(\left(b_{j}^{-1} L_{j}(q)\right)^{p^{m}}\right)
$$

Take the limit as $m \rightarrow \infty$ and the higher order terms will converge to zero. Hence the expression converges to $c_{1} \tau \partial_{1}(y)+\cdots+c_{r} \tau \partial_{r}(y)$.

Therefore $\left(c_{1} \tau \partial_{1}+\cdots+c_{r} \tau \partial_{r}\right)(P)=0$.

Now, using Proposition 2.4 we know that if $\tau \partial_{i}(P)=0$ for some $i \leq r$ then $P$ is controlled by a proper open subgroup of $G$. So we will suppose, for contradiction, that $\tau \partial_{i}(P) \neq 0$ for all $i \leq r$.

Let $N:=\left\{\left(q_{1}, \cdots, q_{r}\right) \in \widehat{Q\left(\frac{k G}{P}\right)}:\left(q_{1} \tau \partial_{1}+\cdots+q_{r} \tau \partial_{r}\right)(P)=0\right\}$, then $0 \neq\left(c_{1}, \cdots, c_{r}\right) \in N$, so $N \neq 0$.

Therefore, using Proposition 6.7, we see that $c_{1}, \cdots, c_{r}$ are $Z(Q)$-linearly dependent.

So, we can find some $1<t \leq r$ such that $c_{1}, \cdots, c_{t}$ are $Z(Q)$-linearly dependent, but no proper subset of $\left\{c_{1}, \cdots, c_{t}\right\}$ is $Z(Q)$-linearly dependent.

It follows that we can find $\alpha_{2}, \cdots, \alpha_{t} \in Z(Q) \backslash\{0\}$ such that $c_{1}+\alpha_{2} c_{2}+\cdots+\alpha_{t} c_{t}=0$.

Therefore, since $c_{i}^{p}=c_{i}$ for all $i$, we also have that:

$$
c_{1}+\alpha_{2}^{p} c_{2}+\cdots+\alpha_{t}^{p} c_{t}=\left(c_{1}+\alpha_{2} c_{2}+\cdots+\alpha_{t} c_{t}\right)^{p}=0
$$

Hence $\left(\alpha_{2}^{p}-\alpha_{2}\right) c_{2}+\cdots+\left(\alpha_{t}^{p}-\alpha_{t}\right) c_{t}=0$.

So using minimality of $\left\{c_{1}, \cdots, c_{t}\right\}$, this means that $\alpha_{i}^{p}=\alpha_{i}$ for each $i$, and it follows that $\alpha_{i} \in \mathbb{F}_{p}$ for each $i$, i.e. $c_{1}, \cdots, c_{r}$ are $\mathbb{F}_{p}$-linearly dependent.

So, we can find $\beta_{1}, \cdots, \beta_{r} \in \mathbb{F}_{p}$, not all zero, such that $\beta_{1} c_{1}+\cdots+\beta_{r} c_{r}=0$, or in other words:

$$
\lim _{m \rightarrow \infty}\left(L_{j}\left(\beta_{1} q_{1}+\cdots+\beta_{r} q_{r}\right)^{p^{k}} b_{j}^{-p^{k}}\right)^{p^{m}}=0
$$


Therefore, $\rho\left(L_{j}\left(\beta_{1} q_{1}+\cdots+\beta_{r} q_{r}\right)^{p^{k}} b_{j}^{-p^{k}}\right)>0$, and hence $\rho\left(L_{j}\left(\beta_{1} q_{1}+\cdots+\beta_{r} q_{r}\right)\right)>v\left(b_{j}\right)=p^{s-j} \lambda$.

But $\beta_{1} q_{1}+\cdots+\beta_{r} q_{r}=\tau\left(u\left(h_{1}^{\beta_{1}} \cdots h_{r}^{\beta_{r}}\right)-1\right)+\varepsilon$, where $\rho(\varepsilon)>\lambda$, and we know that $\rho\left(L_{j}\left(\tau\left(u\left(h_{1}^{\beta_{1}} \cdots h_{r}^{\beta_{r}}\right)-1\right)\right)\right)=$ $p^{s-j} \lambda$ by the definition of $K_{j}$, and $\rho\left(L_{j}(\varepsilon)\right)>p^{s-j} \lambda$ since $L_{j}$ is a GPP.

Hence $\rho\left(L_{j}\left(\beta_{1} q_{1}+\cdots+\beta_{r} q_{r}\right)\right)=\rho\left(L_{j}\left(\tau\left(u\left(h_{1}^{\beta_{1}} \cdots h_{r}^{\beta_{r}}\right)-1\right)\right)\right)=p^{s-j} \lambda-$ contradiction.

Therefore $P$ is controlled by a proper open subgroup of $G$.

\subsection{Control Theorem}

We may now suppose that $L_{i}$ is not trivial for all $i \leq s$, in particular, $L_{0}$ is a non-trivial GPP of $p$-degree $s$.

Proposition 6.10. $L_{0}$ is a special growth preserving polynomial.

Proof. Given $h \in H$ such that $\rho\left(L_{0}(\tau(u(h)-1))\right)=p^{s} \lambda$, we want to prove that $L_{0}(\tau(u(h)-1))^{p^{k}}$ is $v$-regular for some $k$. Let $T=u_{c}(h)-1+F_{\theta+1} k G \in \operatorname{Span}_{\mathbb{F}_{p}}\left\{T_{1}, \cdots, T_{r}\right\}$.

We know that $L^{(s)}\left(\bar{T}, \bar{B}_{s}, \cdots, \bar{B}_{1} p^{p_{1}}\right.$ lies in $A^{\prime}$ by Lemma 6.2 If $L^{(s)}\left(\bar{T}, \bar{B}_{s}, \cdots, \bar{B}_{1}\right)^{p^{m_{0}}} \in \mathfrak{q}$ then we may assume that $L^{(s)}\left(\bar{T}, \bar{B}_{s}, \cdots, \bar{B}_{1}\right)^{p^{m_{1}}}=0$, so using Lemma 6.3 we see that $\bar{w}\left(L^{(s)}\left(\tau(u(h)-1), b_{s}, \cdots, b_{1}\right)\right)>$ $p^{s+m_{1}} \theta=\bar{w}\left(b_{s}^{p^{s}}\right)$.

So again, since $\operatorname{gr}_{\bar{w}}\left(b_{s}\right)=\bar{B}_{s} \in A^{\prime} \backslash \mathfrak{q}$, it follows from Theorem 3.6 that

$$
v\left(L^{(s)}\left(\tau(u(h)-1), b_{s}, \cdots, b_{1}\right)^{p^{m}}\right)>v\left(b_{s}^{p^{m+s}}\right) \text { for } m>>0
$$

Hence $\rho\left(L_{0}(\tau(u(h)-1))\right)=\rho\left(L^{(s)}\left(\tau(u(h)-1), b_{s}, \cdots, b_{1}\right)\right)>\rho\left(b_{s}^{p^{s}}\right) \geq p^{s} \lambda$ - contradiction.

Therefore, we have that $L^{(s)}\left(\bar{T}, \bar{B}_{s}, \cdots, \bar{B}_{1}\right)^{p^{m_{1}}} \in A^{\prime} \backslash q$, and hence it is equal to $\operatorname{gr}_{\bar{w}}\left(L^{(s)}\left(\tau(u(h)-1), b_{s}, \cdots, b_{1}\right)\right)$ by Lemma 6.3 .

It follows from Theorem 3.6 that for $m>>0, L_{0}(\tau(u(h)-1))^{p^{m}}=L^{(s)}\left(\tau(u(h)-1), b_{s}, \cdots, b_{1}\right)^{p^{m}}$ is $v$-regular as required.

Now we can finally prove our main control theorem in all cases. But we first need the following technical result.

Lemma 6.11. Let $G=H \rtimes\langle X\rangle$ be an abelian-by-procyclic group. Then $G$ has split centre if and only if $(G, G) \cap Z(G)=1$.

Proof. It is clear that if $G$ has split centre then $(G, G) \cap Z(G)=1$. Conversely, suppose that $(G, G) \cap Z(G)=1$, and consider the $\mathbb{Z}_{p}$-module homomorphism $H \rightarrow H, h \mapsto(X, h)$.

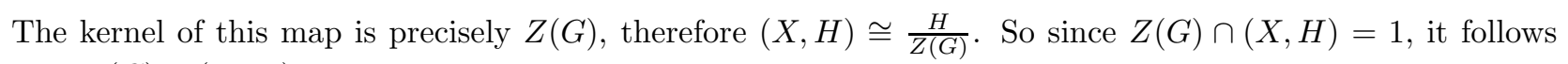
that $Z(G) \times(X, H)$ has the same rank as $H$, hence it is open in $H$.

Recall from [16, Definition 1.6] the definition of the isolator $i_{G}(N)$ of a closed, normal subgroup $N$ of $G$, and recall from [16, Proposition 1.7, Lemma 1.8] that it is a closed, isolated normal subgroup of $G$, and that $N$ is open in $i_{G}(N)$.

Let $C=i_{G}((X, H)) \leq H$, then it is clear that $Z(G) \cap C=1$ and that $Z(G) \times C$ is isolated.

Therefore, since $Z(G) \times(X, H)$ is open in $H$, it follows that $Z(G) \times C=H$, and hence $G=Z(G) \times C \rtimes\langle X\rangle$, and $G$ has split centre.

Proof of Theorem 1.5. Let $Z_{1}(G):=\{g \in G:(g, G) \subseteq Z(G)\}$, this is a closed subgroup of $G$ containing $Z(G)$ and contained in $H$. Suppose first that $Z_{1}(G) \neq Z(G)$.

Then choose $h \in Z_{1}(G) \backslash Z(G)$, then $(h, G) \subseteq Z(G)$, so if we take $\psi \in \operatorname{Inn}(G)$ to be conjugation by $h$, then $\psi$ is trivial mod centre, and clearly $\psi(P)=P$. So it follows that $P$ is controlled by a proper, open subgroup of $G$ by [1, Theorem B]. 
So from now on, we may assume that $Z_{1}(G)=Z(G)$.

Suppose that $(X, h) \in Z(G)$ for some $h \in H$, then clearly $(h, G) \subseteq Z(G)$, so $h \in Z_{1}(G)=Z(G)$, giving that $(X, h)=1$. It follows that $Z(G) \cap(G, G)=1$, and hence $G$ has split centre by Lemma 6.11

Therefore, using Theorem [5.5, we can choose a $k$-basis $\left\{k_{1}, \cdots, k_{d}\right\}$ for $H$ and a filtration $w$ on $k G$ such that $\operatorname{gr}_{w} k G \cong k\left[T_{1}, \cdots, T_{d+1}\right] * \frac{G}{c(G)}$, where $T_{i}=\operatorname{gr}\left(u_{c}\left(k_{i}\right)-1\right)$ for $i \leq r, T_{i}=\operatorname{gr}\left(k_{i}-1\right)$ for $i>r, T_{r}, \cdots, T_{d}$ are central and $\bar{X} T_{i} \bar{X}^{-1}=T_{i}+D_{i}$ for some $D_{i} \in \operatorname{Span}_{\mathbb{F}_{p}}\left\{T_{i+1}, \cdots, T_{r}\right\}$ for all $i<r$.

If $Q\left(\frac{k G}{P}\right)$ is a CSA, then the result follows from Corollary 4.8, so we may assume that $Q\left(\frac{k G}{P}\right)$ is not a CSA.

Hence if each $D_{i}$ is nilpotent mod gr $P$, then $i d: \tau(k H) \rightarrow \tau(k H)$ is a special GPP with respect to some non-commutative valuation by Proposition 5.8

Therefore, by Theorem 3.12, $P$ is controlled by a proper open subgroup of $G$ as required.

If $D_{s}$ is not nilpotent mod gr $P$ for some $s<r$, then we can construct GPP's $L_{s}, \cdots, L_{0}$ with respect to some non-commutative valuation using Proposition 6.5. and $L_{s}$ is non-trivial.

If $L_{j-1}$ is trivial and $L_{j}$ is non-trivial for some $0<j \leq s$, then the result follows from Theorem 6.9. Whereas if all the $L_{i}$ are non-trivial, then $L_{0}$ is a special GPP by Proposition 6.10, and the result follows again from Theorem 3.12

\section{References}

[1] K. Ardakov, Prime ideals in nilpotent Iwasawa algebras. Inventiones Mathematicae 190(2), 439-503 (2012).

[2] K. Ardakov, The Controller Subgroup of One-sided ideals in Completed Group Rings. Contemporary Mathematics, vol 562 (2012).

[3] K. Ardakov; K. A. Brown, Ring-theoretic properties of Iwasawa algebras: a survey. Documenta Math., Extra Volume Coates, 7-33 (2006).

[4] K. Ardakov; F. Wei; J.J. Zhang, Nonexistence of reflexive ideals in Iwasawa algebras of Chevalley type. J. Algebra 320(1), 259-275 (2008).

[5] G.M. Bergmann, A Weak Nullensatz for Valuations. Proceedings of the American Mathematical society 28(1), 32-38 (1971).

[6] T. Diagana; F. Ramaroson, Non-Archimedian Operator Theory. Springer, Heidelberg, (2016).

[7] J.D. Dixon; M.P.F. du Sautoy; A. Mann; D. Segal, Analytic Pro-p Groups (second edition). Cambridge Studies in Advanced Mathematics, vol 61. Cambridge University Press, Cambridge, (1999).

[8] P.M. Eakin, Jr, The converse to a well-known theorem on Noetherian rings. Math. Ann. 177, 1360(37), 278-282 (1968).

[9] N. Jacobson, Basic Algebra 2 (second edition). Dover Publications, (2009).

[10] M. Lazard, Groupes analytiques p-adiques. Publ. Math. IHÉS 26, 389-603 (1965).

[11] H. Li, Lifting Ore sets of Noetherian filtered rings and applications. J. Algebra 179(3), 686-703 (1996).

[12] H. Li; F. Van Oystaeyen, Zariskian Filtrations. Kluwer Academic, Dordrecht, (1996).

[13] J.C. McConnell; J.C. Robson, Noncommutative Noetherian Rings. Graduate Studies in Mathematics, vol 30. American Mathematical Society, Providence, (2001).

[14] D.S. Passman, Infinite Crossed Products. Pure and Applied Mathematics, vol. 135. Academic Press, Boston, (1989).

[15] P. Schneider, p-adic Lie Groups. Grundlehren der Mathematischen Wissenschaften, vol. 344. Springer, Heidelberg, (2011).

[16] W. Woods, On the structure of virtually nilpotent compact $p$-adic analytic groups. arxiv: 1608.03137vl [math.GR], (2016). 